\title{
Asymmetric Arylation of Imines Catalyzed by Heterogeneous Chiral Rhodium Nanoparticles
}

Tomohiro Yasukawa, Tatsuya Kuremoto, Hiroyuki Miyamura, Shū Kobayashi* Department of Chemistry, School of Science, The University of Tokyo, Hongo, Bunkyo-ku, Tokyo 113-0033, Japan.

\section{Supporting Information}

\section{Contents}

1. General-

2. Asymmetric 1,4-addition of arylboronic acids to unsaturated amides$-\mathrm{S}-3$

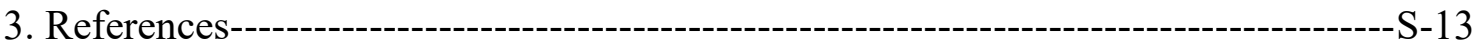

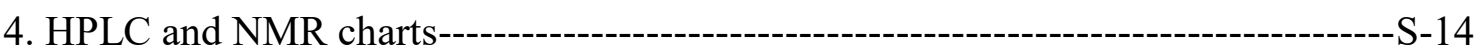

\section{General}

- Melting points were determined on a standard melting point apparatus and are uncorrected.

- JEOL JNM-ECX400, ECA500 or ECX600 spectrometers were used for NMR measurements. Chloroform ( $\delta=7.24$ ) was used as an internal standard for ${ }^{1} \mathrm{H}$ NMR and $\mathrm{CDCl}_{3}(\delta=77.0)$ for ${ }^{13} \mathrm{C}$ NMR. Structures of known compounds were confirmed by comparison with commercially available compounds or data shown in literature.

- IR spectra were measured on a JASCO FT/IR-4200 spectrometer.

- Specific rotations were recorded with JASCO P-1010 or P-2100.

- Preparative thin-layer chromatography was carried out using Wakogel B-5F.

- ICP analysis was performed on Shimadzu ICPS-7510 equipment.

- HPLC analysis was performed on Shimadu LC-20AB, SPD-M20A (or SPD-20A) and DGU-20A 3 .

- The absolute configuration of reported compounds was determined by comparison to literature and that of other products was assumed by analogy.

- $\mathrm{Rh}_{2}(\mathrm{OAc})_{4}$ was purchased from Strem Chemical Inc.

- $\mathrm{AgSbF}_{6}$ was purchased from Sigma-Aldrich Co., Ltd..

- $\mathrm{NaBH}_{4}$ was purchased from Wako Pure Chemical Company and recrystallized from diglyme by heating and stored in a glove box. It is important to manipulate all operations under Ar atmosphere during recrystallization. 
- Ketjen black EC300J was purchased from Lion Corporation.

- Toluene was purchased in dried grade from Wako Pure Chemical Company and used without further purification.

- Deionized water from a MILLIPORE MilliQ machine (Gradient A 10) was used as solvent without further treatment.

- Ethanol was distilled over Na and dried over MS3A.

- Other solvents were purchased in dried grade from Wako Pure Chemical Company and used without further purification.

- Arylboronic acids were purchased from Wako Pure Chemical Company or prepared from the corresponding Grignard reagent. All the arylboronic acids were dehydrated with Dean-Stark apparatus to lead the corresponding arylboroxine-major arylboronic reagents and recrystallized from hexane/ethyl acetate. This method can efficiently remove phenol type impurity. ${ }^{[1]}$ The ratio of boronic acid to boroxine was determined by ${ }^{1} \mathrm{H}$ NMR analysis before use.

- Arylimines were synthesized by condensation of tosylamide and the corresponding aldehydes with Dean-Stark apparatus and recrystallized from hexane/ethyl acetate. Aliphatic imines were prepared by following the literature. ${ }^{[2]}$

- Chiral diene $\mathbf{4}$ was prepared by following the literatures. ${ }^{[3]}$

- $\mathrm{PI} / \mathrm{CB} \mathrm{Rh} / \mathrm{Ag}$ was prepared by following the literature. ${ }^{[4]}$

- Asymmetric arylations were conducted with Carousel $^{\mathrm{TM}}$.

- Unless otherwise stated, all reactions were carried out under argon atmosphere. 


\section{Asymmetric arylation to imines}

Scheme S1. Preparation of PI/CB Rh/Ag catalyst ${ }^{[4]}$

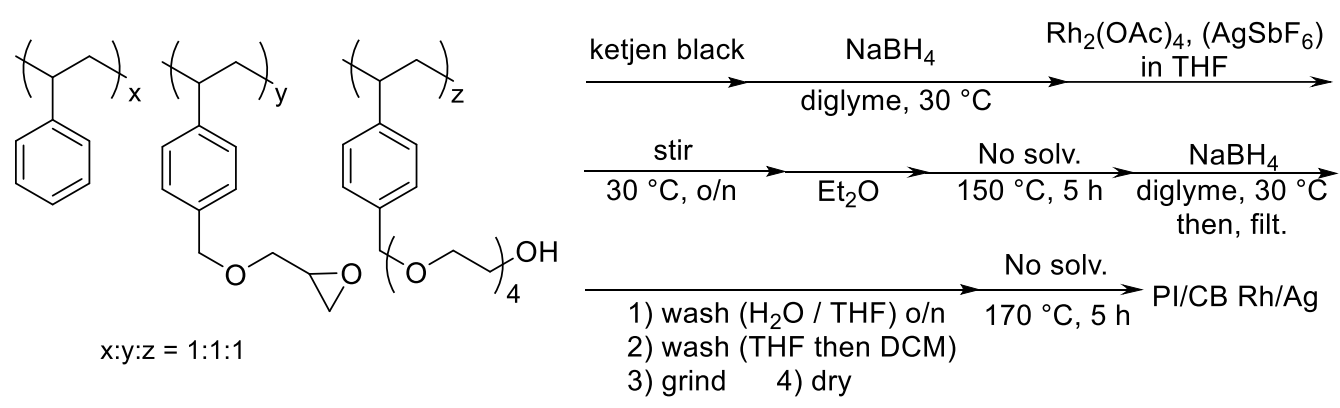

\section{General procedure for preparation of aliphatic imines. ${ }^{[2]}$}

The aldehyde $(10 \mathrm{mmol})$, tosylamide $(10 \mathrm{mmol})$ and sodium $p$-toluenesulfinate (11 mmol) were dissolved in formic acid $(15 \mathrm{~mL})$ and $\mathrm{H}_{2} \mathrm{O}(15 \mathrm{~mL})$. After stirring at room temperature for $12 \mathrm{~h}$, the resulting precipitate was filtered, washed with $\mathrm{H}_{2} \mathrm{O}$, then hexane and dissolved in dichloromethane $(100 \mathrm{~mL})$. The solution and saturated aqueous $\mathrm{NaHCO}_{3}(70 \mathrm{~mL})$ were shaken violently for $45 \mathrm{~s}$. The organic phase was separated, the aqueous phase was extracted with dichloromethane $(70 \mathrm{~mL})$, the combined organic layers were dried over $\mathrm{Na}_{2} \mathrm{SO}_{4}$ and the solvent was removed in vacuo. The residue was purified by recrystallization using hexane/ethyl acetate to afford the corresponding imine.

\section{General procedure for preparation of arylboronic acids.}

Magnesium (33 mmol) was added to THF $(2 \mathrm{~mL})$ and the arylbromide $(30 \mathrm{mmol})$ in THF $(20 \mathrm{~mL})$ was added dropwise to this solution. After stirring for $3 \mathrm{~h}$ at room temperature, the mixture was transferred to a dropping funnel by cannula and added dropwise to trimethyl borate $(36 \mathrm{mmol})$ in THF $(20 \mathrm{~mL})$ at $-78{ }^{\circ} \mathrm{C}$. The mixture was stirred overnight at room temperature. After quenching with $1 \mathrm{M} \mathrm{HCl} \mathrm{aq.,} \mathrm{the} \mathrm{mixture}$ was extracted with dichloromethane, and the organic layers were dried over $\mathrm{Na}_{2} \mathrm{SO}_{4}$ and the solvent was removed in vacuo. The residue was dissolved in toluene and refluxed with Dean-Stark apparatus for $1 \mathrm{~h}$. After the removal of solvent, the residue was purified by recrystallization using hexane/ethyl acetate to afford the corresponding arylboronic acids.

Representative procedure of asymmetric arylation of imines catalyzed by PI/CB Rh/Ag catalyst. (Table 2, entry 1) 
PI/CB Rh/Ag (14.3 mg, Rh: $0.5 \mathrm{~mol} \%)$ and $\mathrm{H}_{2} \mathrm{O}(375 \mu \mathrm{L})$ were combined. To this suspension, arylboronic acid 2a (the mixture of boroxine and boronic acid, $93.9 \mathrm{mg}, 0.9$ mmol as B), imine 1a (122.5 mg, $0.45 \mathrm{mmol}$ ), the toluene solution of chiral diene 4 (90 $\mu \mathrm{L}$ of $2.6 \mathrm{mg} / \mathrm{mL}$ solution of toluene, $0.1 \mathrm{~mol} \%)$ and toluene $(2910 \mu \mathrm{L})$ were added in this order. After the mixture was stirred for $16 \mathrm{~h}$ under $\mathrm{Ar}$ atmosphere at $100{ }^{\circ} \mathrm{C}$, the resulting mixture was cooled to room temperature. The mixture was diluted with $\mathrm{Et}_{2} \mathrm{O}$ and filtered through cotton plug to remove the residual solids. The solution was washed with saturated aqueous $\mathrm{Na}_{2} \mathrm{CO}_{3}$ twice and dried over $\mathrm{Na}_{2} \mathrm{SO}_{4}$, and the solvent was removed in vacuo. The conversion was determined by ${ }^{1} \mathrm{H}$ NMR analysis with reference to an internal standard (1,1,2,2-tetrachloroethane). The crude product was purified by pTLC (hexane/ethyl acetate $=3: 1)$ to afford the pure product 3aa $(136.5 \mathrm{mg}, 87 \%$ yield) . The ee value of the product was determined by chiral HPLC analysis $(99 \%)$.

\section{Representative procedure for preparation of racemic samples. (3aa)}

Imine 1a $(82.4 \mathrm{mg}, 0.3 \mathrm{mmol})$, arylboronic acid 2a (the mixture of boroxine and boronic acid, $68.2 \mathrm{mg}, 0.6 \mathrm{mmol}$ as $\mathrm{B})$ and $\left[\mathrm{Rh}(\mathrm{nbd})_{2}\right]\left(\mathrm{BF}_{4}\right)(10.2 \mathrm{mg}, 10 \mathrm{~mol} \%)$ were dissolved in toluene $(900 \mu \mathrm{L})$ and $\mathrm{H}_{2} \mathrm{O}(225 \mu \mathrm{L})$. After the mixture was stirred for $16 \mathrm{~h}$ under $\mathrm{Ar}$ atmosphere at $100{ }^{\circ} \mathrm{C}$, the resulting mixture was cooled to room temperature. The mixture was diluted with $\mathrm{Et}_{2} \mathrm{O}$, washed with saturated aqueous $\mathrm{Na}_{2} \mathrm{CO}_{3}$ twice and dried over $\mathrm{Na}_{2} \mathrm{SO}_{4}$, and the solvent was removed in vacuo. The crude product was purified by pTLC (hexane/ethyl acetate $=3: 1)$ to afford pure product 3aa $(60.4 \mathrm{mg}, 58 \%$ yield).

\section{Representative procedure for pre-heating experiments. (Table 2, entry 19)}

$\mathrm{PI} / \mathrm{CB} \mathrm{Rh} / \mathrm{Ag}$ (14.3 mg, Rh: $0.5 \mathrm{~mol} \%)$ and $\mathrm{H}_{2} \mathrm{O}(375 \mu \mathrm{L})$ were combined. To this suspension, arylboronic acid $\mathbf{2 a}$ (the mixture of boroxine and boronic acid, $93.9 \mathrm{mg}, 0.9$ mmol as B) and toluene $(750 \mu \mathrm{L})$ were added. After the mixture was stirred for $6 \mathrm{~h}$ under Ar atmosphere at $100{ }^{\circ} \mathrm{C}$, imine $1 \mathrm{~m}(107.7 \mathrm{mg}, 0.45 \mathrm{mmol})$, the toluene solution of chiral diene $4(90 \mu \mathrm{L}$ of $2.6 \mathrm{mg} / \mathrm{mL}$ solution of toluene, $0.1 \mathrm{~mol} \%)$ and toluene $(2160$ $\mu \mathrm{L})$ were added to the resulting mixture. After the mixture was stirred for another $16 \mathrm{~h}$ under $\mathrm{Ar}$ atmosphere at $100{ }^{\circ} \mathrm{C}$, the resulting mixture was cooled to room temperature. The mixture was diluted with $\mathrm{Et}_{2} \mathrm{O}$ and filtered through cotton plug to remove the residual solids. The solution was washed with saturated aqueous $\mathrm{Na}_{2} \mathrm{CO}_{3}$ twice and dried over $\mathrm{Na}_{2} \mathrm{SO}_{4}$, and the solvent was removed in vacuo. The conversion was determined by ${ }^{1} \mathrm{H}$ NMR analysis with reference to an internal standard (1,1,2,2-tetrachloroethane). The crude product was purified by pTLC (hexane/ethyl 
acetate $=3: 1)$ to afford the pure product $3 \mathrm{ma}(47 \mathrm{mg}, 33 \%$ yield). The ee value of the product was determined by chiral HPLC analysis.

\section{Representative procedure of recovery and reuse experiments. (Table 3)}

$\mathrm{PI} / \mathrm{CB} \mathrm{Rh} / \mathrm{Ag}$ (58.5 mg, Rh: $0.5 \mathrm{~mol} \%$ ) and $\mathrm{H}_{2} \mathrm{O}(1.5 \mathrm{~mL})$ were combined. To this suspension, arylboronic acid 2a (the mixture of boroxine and boronic acid, $375.1 \mathrm{mg}$, $3.6 \mathrm{mmol}$ as B), imine 1a (492.1 mg, $1.8 \mathrm{mmol})$, toluene solution of chiral diene 4 (360 $\mu \mathrm{L}$ of $2.6 \mathrm{mg} / \mathrm{mL}$ solution of toluene, $0.1 \mathrm{~mol} \%)$ and toluene $(11.64 \mathrm{~mL})$ were added in this order. After the mixture was stirred for $16 \mathrm{~h}$ under $\mathrm{Ar}$ atmosphere at $100{ }^{\circ} \mathrm{C}$, the resulting mixture was cooled to room temperature. The catalyst was collected by filtration using a KIRIYAMAROHTO ${ }^{\circledR}$ funnel. The remaining PI/CB Rh/Ag on funnel was washed with $\mathrm{H}_{2} \mathrm{O}$, tetrahydrofuran, dichloromethane and dried in vacuo (typically, $90 \mathrm{wt} \%$ of the catalyst is recovered). The filtrate was diluted with $\mathrm{Et}_{2} \mathrm{O}$ and the solution was washed with saturated aqueous $\mathrm{Na}_{2} \mathrm{CO}_{3}$ twice and dried over $\mathrm{Na}_{2} \mathrm{SO}_{4}$, and the solvent was removed in vacuo. The conversion was determined by ${ }^{1} \mathrm{H} \mathrm{NMR}$ analysis with reference to an internal standard (1,1,2,2-tetrachloroethane). The crude product was dissolved in DCM (exactly $10 \mathrm{~mL}$ ). Exactly $1 \mathrm{~mL}$ of the solution was purified with pTLC (hexane/ethyl acetate $=3: 1$ ) to afford the pure product 3aa $(53.6 \mathrm{mg}, 85 \%$ yield). The ee value of the product was determined by chiral HPLC analysis (99\% ee). The recovered catalyst was heated at $150{ }^{\circ} \mathrm{C}$ for $6 \mathrm{~h}$ before use.

\section{(S)-4-methyl- $N$-(phenyl( $p$-tolyl)methyl)benzenesulfonamide (3aa) ${ }^{[5]}$ :}

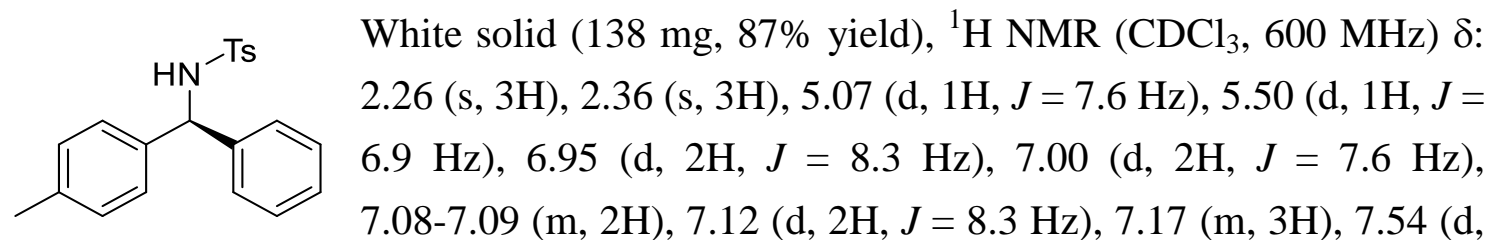

$2 \mathrm{H}, J=4.1 \mathrm{~Hz}) .{ }^{13} \mathrm{C} \mathrm{NMR}\left(\mathrm{CDCl}_{3}, 150 \mathrm{MHz}\right) \delta: 21.0,21.5,61.1,127.19,127.25$, $127.28,128.5,129.2,129.3,137.3,137.5,137.6,143.1$. The ee value of the product was determined on Daicel Chiralcel OD-H column with hexane/2-propanol $=19 / 1$, flow $=$ $1.0 \mathrm{~mL} / \mathrm{min}$ by HPLC analysis. Retention times: $19.4 \mathrm{~min}[(S)$-enamtiomer], $28.6 \mathrm{~min}$ [ $(R)$-enantiomer], $99 \%$ ee.

$(R)$-4-methyl- $N$-(phenyl $\left(p\right.$-tolyl)methyl)benzenesulfonamide $\left(3 \mathrm{hb}=\right.$ ent-3aa $^{[5]}$ : White solid (119 $\mathrm{mg}, 75 \%$ yield), $99 \%$ ee. 
(S)-4-methyl- $N$-(phenyl( $m$-tolyl)methyl)benzenesulfonamide (3ba $)^{[6]}$ :

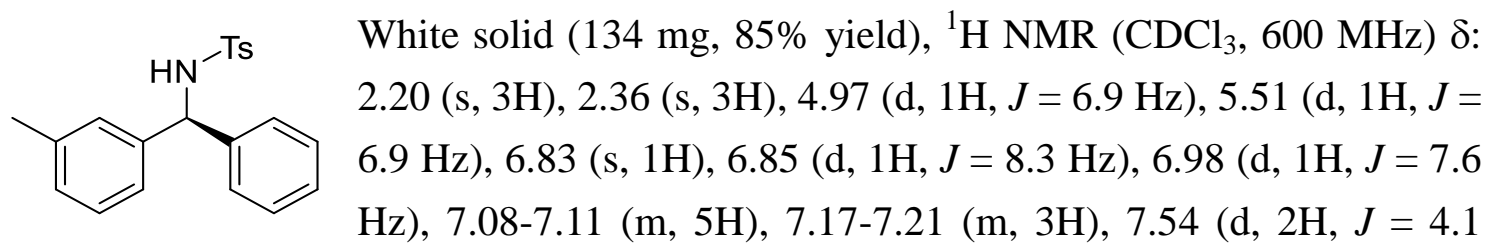

$\mathrm{Hz}) .{ }^{13} \mathrm{C} \mathrm{NMR}\left(\mathrm{CDCl}_{3}, 150 \mathrm{MHz}\right) \delta: 21.3,21.5,61.3,124.4,127.2,127.3,127.5,128.0$, $128.3,128.4,128.5,129.3,138.2,140.3,140.5,134.1$. The ee value of the product was determined on Daicel Chiralcel OD-H column with hexane/2-propanol $=19 / 1$, flow $=$ $1.0 \mathrm{~mL} / \mathrm{min}$ by HPLC analysis. Retention times: $13.7 \mathrm{~min}[(R)$-enamtiomer], $15.1 \mathrm{~min}$ [(S)-enantiomer], $99 \%$ ee.

(S)-4-methyl- $N$-(phenyl $\left(o\right.$-tolyl)methyl)benzenesulfonamide $(3 \mathrm{ca})^{[5]}$ :<smiles>Cc1ccccc1C(NC(F)(F)F)c1ccccc1</smiles>

White solid (144 mg, 91\% yield), ${ }^{1} \mathrm{H}$ NMR $\left(\mathrm{CDCl}_{3}, 600 \mathrm{MHz}\right) \delta: 2.14$ $(\mathrm{s}, 3 \mathrm{H}), 2.35(\mathrm{~s}, 3 \mathrm{H}), 5.10(\mathrm{~d}, 1 \mathrm{H}, J=6.9 \mathrm{~Hz}), 5.78(\mathrm{~d}, 1 \mathrm{H}, J=6.9 \mathrm{~Hz})$, 7.03-7.04 (m, 4H), 7.09-7.10 (m, 4H), 7.16-7.17 (m, 3H), $7.53(\mathrm{~d}, 2 \mathrm{H}$, $J=8.3 \mathrm{~Hz}) .{ }^{13} \mathrm{C} \mathrm{NMR}\left(\mathrm{CDCl}_{3}, 150 \mathrm{MHz}\right) \delta: 19.3,21.4,58.0,126.1$, $127.1,127.2$, 127.5, 128.5, 129.3, 130.6, 135.4, 137.4, 138.2, 139.9, 143.1. The ee value of the product was determined on Daicel Chiralcel OD-H column with hexane $/ 2$-propanol $=19 / 1$, flow $=1.0 \mathrm{~mL} / \mathrm{min}$ by HPLC analysis. Retention times: 14.6 $\min [(R)$-enamtiomer $], 19.7 \min [(S)$-enantiomer $], 96 \%$ ee.

(S)-N-((4-fluorophenyl)(phenyl)methyl)-4-methylbenzenesulfonamide (3da) ${ }^{[7]}$ :<smiles>Fc1ccc(C(NC(F)(F)F)c2ccccc2)cc1</smiles>
White solid (126 mg, 79\% yield), ${ }^{1} \mathrm{H} \mathrm{NMR}\left(\mathrm{CDCl}_{3}, 600 \mathrm{MHz}\right) \delta$ : $2.37(\mathrm{~s}, 3 \mathrm{H}), 5.08(\mathrm{~d}, 1 \mathrm{H}, J=6.9 \mathrm{~Hz}), 5.53(\mathrm{~d}, 1 \mathrm{H}, J=7.6 \mathrm{~Hz})$, $6.87(\mathrm{t}, 2 \mathrm{H}, J=6.4), 7.03-7.08(\mathrm{~m}, 4 \mathrm{H}), 7.13(\mathrm{~d}, 2 \mathrm{H}, J=7.6 \mathrm{~Hz})$, 7.19-7.20 (m, 3H), $7.53(\mathrm{~d}, 2 \mathrm{H}, J=8.3 \mathrm{~Hz}) .{ }^{13} \mathrm{C} \mathrm{NMR}\left(\mathrm{CDCl}_{3}, 150\right.$ MHz) $\delta: 21.5,60.7,115.3(\mathrm{~d}, 2 \mathrm{C}, J=21.5 \mathrm{~Hz}), 127.15,127.23,127.7,128.6,129.0$, $129.1,129.4,136.3$ (d, 1C, $J=2.9 \mathrm{~Hz}), 137.2,140.3,143.3,162.0(\mathrm{~d}, 1 \mathrm{C}, J=245.7 \mathrm{~Hz})$. The ee value of the product was determined on Daicel Chiralcel OD-H column with hexane $/ 2$-propanol $=19 / 1$, flow $=1.0 \mathrm{~mL} / \mathrm{min}$ by HPLC analysis. Retention times: 15.7 $\min [(S)$-enamtiomer], $19.7 \min [(R)$-enantiomer], $99 \%$ ee.

(S)-N-((4-chlorophenyl)(phenyl)methyl)-4-methylbenzenesulfonamide (3ea) ${ }^{[8]}$ :

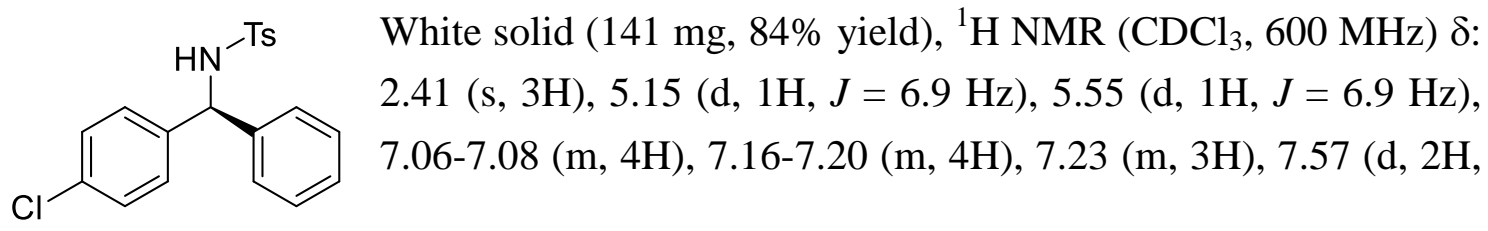


$J=8.3 \mathrm{~Hz}) .{ }^{13} \mathrm{C} \mathrm{NMR}\left(\mathrm{CDCl}_{3}, 150 \mathrm{MHz}\right) \delta: 21.5,60.7,127.2,127.3,127.9,128.6$, $128.7,128.8,129.4,133.5,137.2,138.9,140.0,143.4$. The ee value of the product was determined on Daicel Chiralcel OD-H column with hexane/2-propanol $=19 / 1$, flow $=$ $1.0 \mathrm{~mL} / \mathrm{min}$ by HPLC analysis. Retention times: $20.5 \mathrm{~min}$ [(S)-enamtiomer], $27.6 \mathrm{~min}$ [(R)-enantiomer], $99 \%$ ee.

(S)-N-((4-bromophenyl)(phenyl)methyl)-4-methylbenzenesulfonamide (3fa) ${ }^{[5]}$ : $2.42(\mathrm{~s}, 3 \mathrm{H}), 5.11(\mathrm{~d}, 1 \mathrm{H}, J=7.6 \mathrm{~Hz}), 5.54(\mathrm{~d}, 1 \mathrm{H}, J=6.9 \mathrm{~Hz})$,
$7.02(\mathrm{~d}, 2 \mathrm{H}, J=8.3), 7.07(\mathrm{q}, 2 \mathrm{H}, J=3.2 \mathrm{~Hz}), 7.17(\mathrm{~d}, 2 \mathrm{H}, J=$
$8.3 \mathrm{~Hz}), 7.24(\mathrm{~m}, 3 \mathrm{H}), 7.35(\mathrm{~d}, 2 \mathrm{H}, J=4.1 \mathrm{~Hz}), 7.57(\mathrm{~d}, 2 \mathrm{H}, J=$ $8.3 \mathrm{~Hz}) .{ }^{13} \mathrm{C} \mathrm{NMR}\left(\mathrm{CDCl}_{3}, 150 \mathrm{MHz}\right) \delta: 21.5,60.8,121.6,127.2,127.3,127.9,128.8$, $129.1,129.4,131.6,137.1,139.4,140.0 .143 .5$. The ee value of the product was determined on Daicel Chiralcel OD-H column with hexane/2-propanol $=19 / 1$, flow $=$ $1.0 \mathrm{~mL} / \mathrm{min}$ by HPLC analysis. Retention times: $21.6 \mathrm{~min}$ [(S)-enamtiomer], $29.9 \mathrm{~min}$ $[(R)$-enantiomer], $99 \%$ ee.

(S)-N-((4-methoxyphenyl)(phenyl)methyl)-4-methylbenzenesulfonamide (3ga) ${ }^{[7]}$ :<smiles>COc1ccc(C(NC(F)(F)F)c2ccccc2)cc1</smiles>

White solid (142 mg, 86\% yield), ${ }^{1} \mathrm{H} \mathrm{NMR}\left(\mathrm{CDCl}_{3}, 600 \mathrm{MHz}\right)$ $\delta: 2.36(\mathrm{~s}, 3 \mathrm{H}), 3.73(\mathrm{~s}, 3 \mathrm{H}), 4.96(\mathrm{~d}, 1 \mathrm{H}, J=6.9 \mathrm{~Hz}), 5.50(\mathrm{~d}$, $1 \mathrm{H}, J=6.9 \mathrm{~Hz}), 6.72(\mathrm{~d}, 2 \mathrm{H}, J=8.9 \mathrm{~Hz}), 6.97(\mathrm{~d}, 2 \mathrm{H}, J=8.9$ $\mathrm{Hz}), 7.08(\mathrm{~d}, 2 \mathrm{H}, J=3.8 \mathrm{~Hz}), 7.13(\mathrm{~d}, 2 \mathrm{H}, J=8.3 \mathrm{~Hz})$, 7.17-7.20 (m, 3H), $7.54(\mathrm{~d}, 2 \mathrm{H}, J=8.3 \mathrm{~Hz}) .{ }^{13} \mathrm{C} \mathrm{NMR}\left(\mathrm{CDCl}_{3}, 150 \mathrm{MHz}\right) \delta: 21.5,55.2$, $60.8,113.9,127.20,127.24,128.5,128.6,129.3,132.7,140.7,143.1,159.0$. The ee value of the product was determined on Daicel Chiralcel OD-H column with hexane $/ 2$-propanol $=19 / 1$, flow $=1.0 \mathrm{~mL} / \mathrm{min}$ by HPLC analysis. Retention times: 22.8 $\min [(S)$-enamtiomer], $32.7 \mathrm{~min}[(R)$-enantiomer], $99 \%$ ee.

(R)-4-methyl- $N$-( $m$-tolyl $\left(p\right.$-tolyl)methyl)benzenesulfonamide (3ac) ${ }^{[8]}$ :

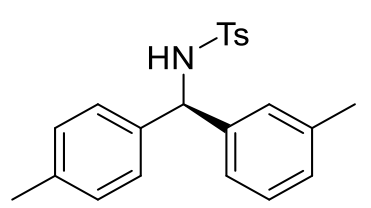

White solid (133 mg, 81\% yield), ${ }^{1} \mathrm{H} \mathrm{NMR}\left(\mathrm{CDCl}_{3}, 600 \mathrm{MHz}\right)$ $\delta: 2.18(\mathrm{~s}, 3 \mathrm{H}), 2.26(\mathrm{~s}, 3 \mathrm{H}), 2.36(\mathrm{~s}, 3 \mathrm{H}), 5.12(\mathrm{~d}, 1 \mathrm{H}, J=6.9$ $\mathrm{Hz}), 5.47(\mathrm{~d}, 1 \mathrm{H}, J=6.9 \mathrm{~Hz}), 6.84(\mathrm{~s}, 1 \mathrm{H}), 6.86(\mathrm{~d}, 1 \mathrm{H}, J=8.3$ $\mathrm{Hz}), 6.96-7.00(\mathrm{~m}, 5 \mathrm{H}), 7.06(\mathrm{t}, 1 \mathrm{H}, J=7.6 \mathrm{~Hz}), 7.11(\mathrm{~d}, 2 \mathrm{H}, J=$ $7.6 \mathrm{~Hz}), 7.53(\mathrm{~d}, 2 \mathrm{H}, J=8.3 \mathrm{~Hz}) .{ }^{13} \mathrm{C} \mathrm{NMR}\left(\mathrm{CDCl}_{3}, 150 \mathrm{MHz}\right) \delta: 21.0,21.3,21.4,61.1$, 124.3, 127.20, 127.22, 127.9, 128.2, 128.4, 129.1, 129.2, 137.2, 137.4, 137.7, 138.1, 140.5, 143.0. The ee value of the product was determined on Daicel Chiralcel OD-H 
column with hexane $/ 2$-propanol $=19 / 1$, flow $=1.0 \mathrm{~mL} / \mathrm{min}$ by HPLC analysis. Retention times: $13.1 \mathrm{~min}[(S)$-enamtiomer], $20.4 \min$ [ $(R)$-enantiomer], 99\% ee.

\section{(R)-4-methyl- $N$-(o-tolyl $(p$-tolyl)methyl)benzenesulfonamide (3ad):}<smiles>[3H]NC(c1ccc(C)cc1)c1ccccc1C</smiles>

White solid (138 mg, 84\% yield), IR (KBr) cm$~^{-1}: 3297,3245,2923$, 2863, 1436, 1316, 1148, 1060, 662. M.p. $=99-102{ }^{\circ} \mathrm{C} .{ }^{1} \mathrm{H}$ NMR $\left(\mathrm{CDCl}_{3}, 600 \mathrm{MHz}\right) \delta: 2.12(\mathrm{~s}, 3 \mathrm{H}), 2.26(\mathrm{~s}, 3 \mathrm{H}), 2.35(\mathrm{~s}, 3 \mathrm{H}), 5.04$ $(\mathrm{d}, 1 \mathrm{H}, J=6.9 \mathrm{~Hz}), 5.73(\mathrm{~d}, 1 \mathrm{H}, J=6.9 \mathrm{~Hz}), 6.91(\mathrm{~d}, 2 \mathrm{H}, J=8.3$ $\mathrm{Hz}), 6.98(\mathrm{~d}, 2 \mathrm{H}, J=8.3 \mathrm{~Hz}), 7.03(\mathrm{~d}, 2 \mathrm{H}, J=7.2 \mathrm{~Hz}), 7.07-7.13(\mathrm{~m}, 4 \mathrm{H}), 7.53(\mathrm{~d}, 2 \mathrm{H}, J$ $=8.3 \mathrm{~Hz}) .{ }^{13} \mathrm{C} \mathrm{NMR}\left(\mathrm{CDCl}_{3}, 150 \mathrm{MHz}\right) \delta: 19.3,20.1,21.4,57.8,126.1,127.08,127.10$, $127.37,127.43,129.19,129.25,130.6,135.3,137.0,137.3,137.4,138.3,143.1$. ESI-MS (m/z) calcd. for $\mathrm{C}_{22} \mathrm{H}_{23} \mathrm{NNaO}_{2} \mathrm{~S}\left([\mathrm{M}+\mathrm{Na}]^{+}\right)$: 388.1347 , found: 388.1351 . The ee value of the product was determined on Daicel Chiralcel OD-H column with hexane/2-propanol $=19 / 1$, flow $=1.0 \mathrm{~mL} / \mathrm{min}$ by HPLC analysis. Retention times: 10.1 $\min \left[(S)\right.$-enamtiomer], $16.6 \min [(R)$-enantiomer $],[\alpha]^{20}{ }_{\mathrm{D}}=-21.4\left(\mathrm{c} 1.08, \mathrm{CHCl}_{3}, 95 \%\right.$ ee).

\section{(R)- $N$-((4-fluorophenyl)(p-tolyl)methyl)-4-methylbenzenesulfonamide (3ae $)^{[8]}$ :}

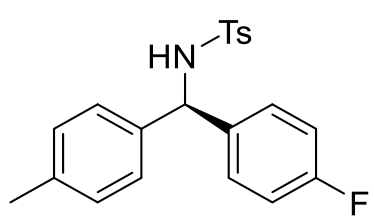

White solid (155 mg, 93\% yield), ${ }^{1} \mathrm{H} \mathrm{NMR}\left(\mathrm{CDCl}_{3}, 600 \mathrm{MHz}\right)$ $\delta: 2.26(\mathrm{~s}, 3 \mathrm{H}), 2.37$ (s, 3H), $5.19(\mathrm{~d}, 1 \mathrm{H}, J=6.9 \mathrm{~Hz}), 5.48(\mathrm{~d}$, $1 \mathrm{H}, J=6.9 \mathrm{~Hz}), 6.86(\mathrm{t}, 2 \mathrm{H}, J=11.7 \mathrm{~Hz}), 6.91(\mathrm{~d}, 2 \mathrm{H}, J=7.6$ $\mathrm{Hz}), 6.99(\mathrm{~d}, 2 \mathrm{H}, J=8.3 \mathrm{~Hz}), 7.07(\mathrm{t}, 2 \mathrm{H}, J=5.8 \mathrm{~Hz}), 7.12(\mathrm{~d}$, $2 \mathrm{H}, J=8.3 \mathrm{~Hz}), 7.53(\mathrm{~d}, 2 \mathrm{H}, J=4.5 \mathrm{~Hz}) \cdot{ }^{13} \mathrm{C} \mathrm{NMR}\left(\mathrm{CDCl}_{3}, 150 \mathrm{MHz}\right) \delta: 21.0,21.4$, $60.4,115.2(\mathrm{~d}, 2 \mathrm{C}, J=21.7 \mathrm{~Hz}), 127.1,128.95,129.00,129.26,129.31,136.47,136.49$, $137.2,137.4,137.5,143.2,161.9(\mathrm{~d}, 1 \mathrm{C}, J=247.1 \mathrm{~Hz})$. The ee value of the product was determined on Daicel Chiralcel OD-H column with hexane/2-propanol $=19 / 1$, flow $=$ $1.0 \mathrm{~mL} / \mathrm{min}$ by HPLC analysis. Retention times: $23.1 \mathrm{~min}[(S)$-enamtiomer], $29.9 \mathrm{~min}$ $[(R)$-enantiomer], $99 \%$ ee.

(R)-N-((4-chlorophenyl)(p-tolyl)methyl)-4-methylbenzenesulfonamide (3af $)^{[8]}$ :<smiles>Cc1ccc(C(NC(F)(F)F)c2ccc(Cl)cc2)cc1</smiles>

White solid (134 mg, 77\% yield), ${ }^{1} \mathrm{H} \mathrm{NMR}\left(\mathrm{CDCl}_{3}, 600 \mathrm{MHz}\right)$ $\delta: 2.27(\mathrm{~s}, 3 \mathrm{H}), 2.38(\mathrm{~s}, 3 \mathrm{H}), 4.93(\mathrm{~d}, 1 \mathrm{H}, J=6.2 \mathrm{~Hz}), 5.47(\mathrm{~d}$, $1 \mathrm{H}, J=6.9 \mathrm{~Hz}), 6.89(\mathrm{~d}, 2 \mathrm{H}, J=8.3 \mathrm{~Hz}), 7.01(\mathrm{~d}, 2 \mathrm{H}, J=8.3$

$\mathrm{Hz}), 7.04(\mathrm{~d}, 2 \mathrm{H}, J=8.3 \mathrm{~Hz}), 7.13-7.16(\mathrm{~m}, 4 \mathrm{H}), 7.53(\mathrm{~d}, 2 \mathrm{H}, J$ $=8.3 \mathrm{~Hz}) .{ }^{13} \mathrm{C} \mathrm{NMR}\left(\mathrm{CDCl}_{3}, 125 \mathrm{MHz}\right) \delta: 20.9,21.3,60.4,127.0,127.1,128.4,128.6$, $129.17,129.21,133.0,137.09,137.14,137.3,139.2,143.1$. The ee value of the product 
was determined on Daicel Chiralcel OD-H column with hexane/2-propanol $=19 / 1$, flow $=1.0 \mathrm{~mL} / \mathrm{min}$ by HPLC analysis. Retention times: $23.2 \mathrm{~min}[(R)$-enamtiomer], $26.8 \mathrm{~min}$ [(S)-enantiomer], $99 \%$ ee.

(R)-N-((4-bromophenyl)(p-tolyl)methyl)-4-methylbenzenesulfonamide (3ag):<smiles>Cc1ccc(C(NS)c2ccc(Br)cc2)cc1</smiles>

White solid (155 mg, 80\% yield), IR (KBr) $\mathrm{cm}^{-1}$ : 3266, 3029, 2948, 2922, 2865, 1912, 1597, 1489, 1443, 1322, 1161, 1051, 807, 575. M.p. $=140-142{ }^{\circ} \mathrm{C} . \quad{ }^{1} \mathrm{H}$ NMR $\left(\mathrm{CDCl}_{3}, 600 \mathrm{MHz}\right) \delta$ : $2.26(\mathrm{~s}, 3 \mathrm{H}), 2.38(\mathrm{~s}, 3 \mathrm{H}), 4.96(\mathrm{~d}, 1 \mathrm{H}, J=6.9 \mathrm{~Hz}), 5.45(\mathrm{~d}, 1 \mathrm{H}$, $J=6.9 \mathrm{~Hz}), 6.89$ (t, 2H, $J=7.6), 6.97-7.01(\mathrm{~m}, 4 \mathrm{H}), 7.14(\mathrm{~d}, 2 \mathrm{H}, J=8.3 \mathrm{~Hz}), 7.30(\mathrm{~d}$, $2 \mathrm{H}, J=8.9 \mathrm{~Hz}), 7.53(\mathrm{~d}, 2 \mathrm{H}, J=8.3 \mathrm{~Hz}) .{ }^{13} \mathrm{C} \mathrm{NMR}\left(\mathrm{CDCl}_{3}, 125 \mathrm{MHz}\right) \delta: 20.9,21.4$, 60.5, 121.2, 127.01, 127.05, 129.0, 129.18, 129.23, 131.3, 137.0, 137.1, 137.3, 139.7, 143.1. ESI-MS (m/z) calcd. for $\mathrm{C}_{21} \mathrm{H}_{20} \mathrm{BrNNaO}_{2} \mathrm{~S}\left([\mathrm{M}+\mathrm{Na}]^{+}\right)$: 452.0296, found: 452.0311 The ee value of the product was determined on Daicel Chiralcel OD-H column with hexane $/ 2$-propanol $=19 / 1$, flow $=1.0 \mathrm{~mL} / \mathrm{min}$ by HPLC analysis. Retention times: $28.5 \min \left[(R)\right.$-enamtiomer], $32.5 \min [(S)$-enantiomer $],[\alpha]^{25}=-2.9$ (c $1.00, \mathrm{CHCl}_{3}, 99 \%$ ee).

(R)-N-((4-methoxyphenyl)(p-tolyl)methyl)-4-methylbenzenesulfonamide $(3 a h)^{[8]}$ :<smiles>COc1ccc(C(NC(F)(F)F)c2ccc(C)cc2)cc1</smiles>
White solid (148 mg, 86\% yield), ${ }^{1} \mathrm{H} \mathrm{NMR}\left(\mathrm{CDCl}_{3}, 600\right.$ MHz) $\delta: 2.26$ (s, 3H), 2.36 (s, 3H), 3.73 (s, 3H), 5.10 (d, 1H, $J=6.9 \mathrm{~Hz}), 5.46(\mathrm{~d}, 1 \mathrm{H}, J=6.9 \mathrm{~Hz}), 6.70(\mathrm{~d}, 2 \mathrm{H}, J=2.8$ $\mathrm{Hz}), 6.95-7.00(\mathrm{~m}, 6 \mathrm{H}), 7.12(\mathrm{~d}, 2 \mathrm{H}, J=8.3 \mathrm{~Hz}), 7.54(\mathrm{~d}, 2 \mathrm{H}$, $J=8.3 \mathrm{~Hz}) .{ }^{13} \mathrm{C} \mathrm{NMR}\left(\mathrm{CDCl}_{3}, 150 \mathrm{MHz}\right) \delta: 21.0,21.4,55.2,60.6,113.8,127.15$, $127.19,128.5,129.1,129.3,132.9,137.2,137.4,137.8,143.0,158.9$. The ee value of the product was determined on Daicel Chiralcel OD-H column with hexane/2-propanol $=19 / 1$, flow $=1.0 \mathrm{~mL} / \mathrm{min}$ by HPLC analysis. Retention times: $37.5 \mathrm{~min}$ [(R)-enamtiomer], $42.2 \min [(S)$-enantiomer], $99 \%$ ee.

(S)-4-methyl- $N$-(naphthalen-1-yl(phenyl)methyl)benzenesulfonamide $(3 i a)^{[7]}$ :<smiles>[As]N[C@H](c1ccccc1)c1cccc2ccccc12</smiles>
White solid (167 mg, 96\% yield), ${ }^{1} \mathrm{H} \mathrm{NMR}\left(\mathrm{CDCl}_{3}, 600 \mathrm{MHz}\right) \delta$ : $2.33(\mathrm{~s}, 3 \mathrm{H}), 5.13(\mathrm{~d}, 1 \mathrm{H}, J=6.9 \mathrm{~Hz}), 6.29(\mathrm{~d}, 1 \mathrm{H}, J=6.9 \mathrm{~Hz})$, $7.04(\mathrm{~d}, 2 \mathrm{H}, J=8.3 \mathrm{~Hz}), 7.12-7.23(\mathrm{~m}, 2 \mathrm{H}), 7.17-7.18(\mathrm{~m}, 3 \mathrm{H})$, $7.22(\mathrm{~d}, 1 \mathrm{H}, J=6.9 \mathrm{~Hz}), 7.27(\mathrm{t}, 1 \mathrm{H}, J=7.6 \mathrm{~Hz}), 7.37(\mathrm{t}, 1 \mathrm{H}, J=$ $7.6 \mathrm{~Hz}), 7.42$ (t, $1 \mathrm{H}, J=7.6 \mathrm{~Hz}), 7.49$ (d, 2H, $J=8.3 \mathrm{~Hz}), 7.71(\mathrm{~d}, 1 \mathrm{H}, J=8.3 \mathrm{~Hz}), 7.79$ $(\mathrm{t}, 2 \mathrm{H}, J=8.9 \mathrm{~Hz}) .{ }^{13} \mathrm{C} \mathrm{NMR}\left(\mathrm{CDCl}_{3}, 150 \mathrm{MHz}\right) \delta: 21.4,58.5,123.4,125.0,125.7$, 
$126.1,126.5,127.1,127.5,127.6,128.56,128.58,128.8,129.2,130.4$, 133.9, 137.2, 140.2, 143.1. The ee value of the product was determined on Daicel Chiralcel OD-H column with hexane/2-propanol $=9 / 1$, flow $=1.0 \mathrm{~mL} / \mathrm{min}$ by HPLC analysis. Retention times: $19.9 \min [(R)$-enamtiomer], $26.7 \min [(S)$-enantiomer], 99\% ee.

(S)-4-methyl- $N$-(naphthalen-2-yl(phenyl)methyl)benzenesulfonamide $(3 \mathbf{j a})^{[8]}$ :<smiles>[NH3+]C(c1ccccc1)c1ccc2ccccc2c1</smiles>

White solid (162 mg, 93\% yield), ${ }^{1} \mathrm{H} \mathrm{NMR}\left(\mathrm{CDCl}_{3}, 600 \mathrm{MHz}\right)$ $\delta: 2.29(\mathrm{~s}, 3 \mathrm{H}), 5.33(\mathrm{~d}, 1 \mathrm{H}, J=6.9 \mathrm{~Hz}), 5.76(\mathrm{~d}, 1 \mathrm{H}, J=7.6 \mathrm{~Hz})$, $7.05(\mathrm{~d}, 2 \mathrm{H}, J=8.3 \mathrm{~Hz}), 7.17-7.21(\mathrm{~m}, 3 \mathrm{H}), 7.24-7.25(\mathrm{~m}, 3 \mathrm{H})$, $7.47(\mathrm{q}, 2 \mathrm{H}, J=3.0 \mathrm{~Hz}), 7.52(\mathrm{~s}, 1 \mathrm{H}), 7.57(\mathrm{~d}, 2 \mathrm{H}, J=8.3 \mathrm{~Hz})$, 7.66-7.67 (m, 1H) $7.69(\mathrm{~d}, 1 \mathrm{H}, J=8.3 \mathrm{~Hz}), 7.77-7.78(\mathrm{~m}, 1 \mathrm{H}) .{ }^{13} \mathrm{C} \mathrm{NMR}\left(\mathrm{CDCl}_{3}, 150\right.$ MHz) $\delta: 21.3,61.4,125.1,126.1,126.2,126.3,127.2,127.4,127.5,127.7,127.9,128.5$, $128.6,129.2,132.6,133.0,137.3,137.5,140.4,143.2$. The ee value of the product was determined on Daicel Chiralcel OD-H column with hexane/2-propanol $=19 / 1$, flow $=$ $1.0 \mathrm{~mL} / \mathrm{min}$ by HPLC analysis. Retention times: $32.4 \mathrm{~min}[(R)$-enamtiomer], $35.3 \mathrm{~min}$ [(S)-enantiomer], $99 \%$ ee.

\section{(S)-N-(furan-2-yl(phenyl)methyl)-4-methylbenzenesulfonamide (3ka) ${ }^{[7]}$ :}

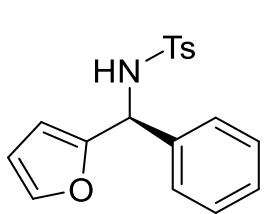

White solid (108 mg, 73\% yield), ${ }^{1} \mathrm{H} \mathrm{NMR}\left(\mathrm{CDCl}_{3}, 600 \mathrm{MHz}\right) \delta: 2.36$ $(\mathrm{s}, 3 \mathrm{H}), 5.24(\mathrm{~d}, 1 \mathrm{H}, J=8.3 \mathrm{~Hz}), 5.59(\mathrm{~d}, 1 \mathrm{H}, J=7.6 \mathrm{~Hz}), 5.97(\mathrm{~d}, 1 \mathrm{H}$, $J=2.8 \mathrm{~Hz}), 6.17(\mathrm{~d}, 1 \mathrm{H}, J=1.8 \mathrm{~Hz}), 7.14-7.15(\mathrm{~m}, 4 \mathrm{H}), 7.21-7.22(\mathrm{~m}$, $3 \mathrm{H}), 7.56(\mathrm{~d}, 2 \mathrm{H}, J=8.3 \mathrm{~Hz}) .{ }^{13} \mathrm{C} \mathrm{NMR}\left(\mathrm{CDCl}_{3}, 150 \mathrm{MHz}\right) \delta: 21.5$, 55.4, 108.4, 110.2, 127.1, 127.2, 128.0, 128.5, 129.3, 137.3, 138.2, 142.6, 143.2, 152.2. The ee value of the product was determined on Daicel Chiralcel OD-H column with hexane $/ 2$-propanol $=19 / 1$, flow $=1.0 \mathrm{~mL} / \mathrm{min}$ by HPLC analysis. Retention times: 20.2 $\min [(S)$-enamtiomer], $22.1 \mathrm{~min}[(R)$-enantiomer], 99\% ee.

\section{(S)-4-methyl- $N$-(phenyl(thiophen-2-yl)methyl)benzenesulfonamide (3la) ${ }^{[5]}$ :}

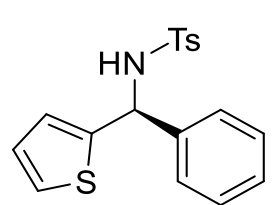

White solid (138 mg, 89\% yield), ${ }^{1} \mathrm{H} \mathrm{NMR}\left(\mathrm{CDCl}_{3}, 600 \mathrm{MHz}\right) \delta: 2.36$ $(\mathrm{s}, 3 \mathrm{H}), 5.23(\mathrm{~d}, 1 \mathrm{H}, J=7.6 \mathrm{~Hz}), 5.77(\mathrm{~d}, 1 \mathrm{H}, J=7.6 \mathrm{~Hz}), 6.65(\mathrm{t}, 1 \mathrm{H}$, $J=1.7 \mathrm{~Hz}), 6.81(\mathrm{q}, 1 \mathrm{H}, J=3.0 \mathrm{~Hz}), 7.14-7.15(\mathrm{~m}, 5 \mathrm{H}), 7.21(\mathrm{t}, 2 \mathrm{H}, J$ $=3.1 \mathrm{~Hz}), 7.56(\mathrm{~d}, 2 \mathrm{H}, J=8.3 \mathrm{~Hz}) \cdot{ }^{13} \mathrm{C} \mathrm{NMR}\left(\mathrm{CDCl}_{3}, 150 \mathrm{MHz}\right) \delta$ :

21.5, 57.4, 125.7, 126.1, 126.7, 127.1, 127.2, 127.9, 128.5, 129.4, 137.2, 140.1, 143.3, 144.8. The ee value of the product was determined on Daicel Chiralcel OD-H column with hexane/2-propanol $=19 / 1$, flow $=1.0 \mathrm{~mL} / \mathrm{min}$ by HPLC analysis. Retention times: $20.8 \mathrm{~min}[(S)$-enamtiomer], $28.8 \mathrm{~min}[(R)$-enantiomer], $99 \%$ ee. 
(R)- $N$-(2,2-dimethyl-1-phenylpropyl)-4-methylbenzenesulfonamide (3ma $)^{[6]}$ :

White solid $(47 \mathrm{mg}, 33 \%$ yield $),{ }^{1} \mathrm{H} \mathrm{NMR}\left(\mathrm{CDCl}_{3}, 600 \mathrm{MHz}\right) \delta: 0.88(\mathrm{~s}$,
$9 \mathrm{H}), 2.25(\mathrm{~s}, 3 \mathrm{H}), 4.00(\mathrm{~d}, 1 \mathrm{H}, J=9.7 \mathrm{~Hz}), 5.88(\mathrm{~d}, 1 \mathrm{H}, J=8.9 \mathrm{~Hz})$,
$6.85(\mathrm{~d}, 2 \mathrm{H}, J=1.4 \mathrm{~Hz}), 6.90(\mathrm{~d}, 2 \mathrm{H}, J=8.3 \mathrm{~Hz}), 7.00-7.05(\mathrm{~m}, 3 \mathrm{H})$,
$7.40(\mathrm{~d}, 2 \mathrm{H}, J=8.2 \mathrm{~Hz}) .{ }^{13} \mathrm{C} \mathrm{NMR}\left(\mathrm{CDCl}_{3}, 150 \mathrm{MHz}\right) \delta: 21.3,26.6$, $35.23,33.9,126.7,127.0,127.4,128.0,128.9,167.2,138.3,142.6$. The ee value of the product was determined on Daicel Chiralcel OD-H column with hexane/2-propanol = 19/1, flow $=1.0 \mathrm{~mL} / \mathrm{min}$ by HPLC analysis. Retention times: $12.2 \mathrm{~min}$ $[(S)$-enamtiomer], $13.2 \mathrm{~min}[(R)$-enantiomer $], 99 \%$ ee.

(R)- $N$-(cyclohexyl(phenyl)methyl)-4-methylbenzenesulfonamide (3na ${ }^{[6]}$ :

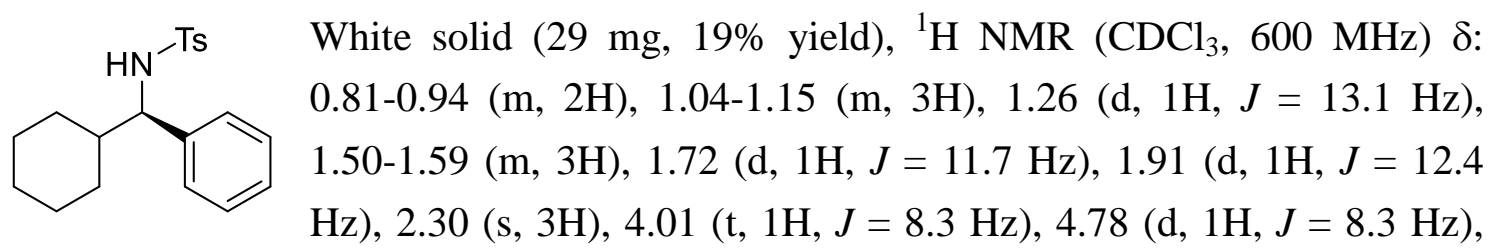
$6.88(\mathrm{q}, 2 \mathrm{H}, J=3.2), 7.02(\mathrm{~d}, 2 \mathrm{H}, J=7.6 \mathrm{~Hz})$ ), 7.07-7.09 (m, 3H), $7.43(\mathrm{t}, 2 \mathrm{H}, J=4.1$ $\mathrm{Hz}) .{ }^{13} \mathrm{C} \mathrm{NMR}\left(\mathrm{CDCl}_{3}, 125 \mathrm{MHz}\right) \delta: 21.4,25.9,26.1,29.4,29.7,43.7,63.4,126.8$, $126.9,127.0,128.0,129.0,137.6,139.9,142.6$. The ee value of the product was determined on Daicel Chiralcel OD-H column with hexane/2-propanol $=19 / 1$, flow $=$ $1.0 \mathrm{~mL} / \mathrm{min}$ by HPLC analysis. Retention times: $9.9 \mathrm{~min}$ [(R)-enamtiomer], $21.4 \mathrm{~min}$ [(S)-enantiomer], $99 \%$ ee.

(S)-4-nitro- $N$-(phenyl( $p$-tolyl)methyl)benzenesulfonamide $(30 a)^{[8]}$ :

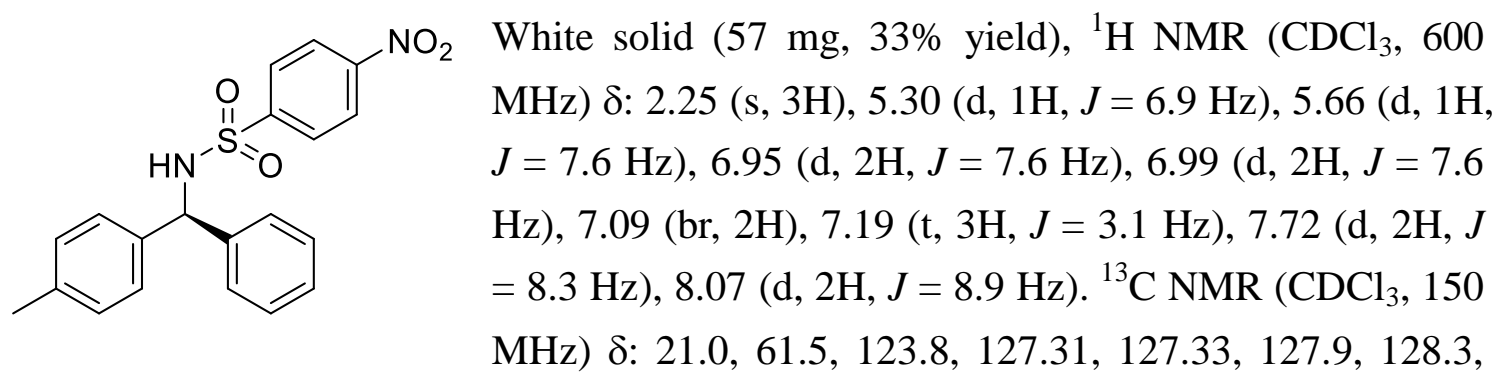

$128.7,129.4,136.5,138.0,139.6,146.3,149.6$. The ee value of the product was determined on Daicel Chiralcel OD-H column with hexane/2-propanol $=9 / 1$, flow $=1.0$ $\mathrm{mL} / \mathrm{min}$ by HPLC analysis. Retention times: $24.2 \mathrm{~min}[(S)$-enamtiomer], $37.0 \mathrm{~min}$ [(R)-enantiomer], $99 \%$ ee. 
Table S1. Screening of inorganic bases.

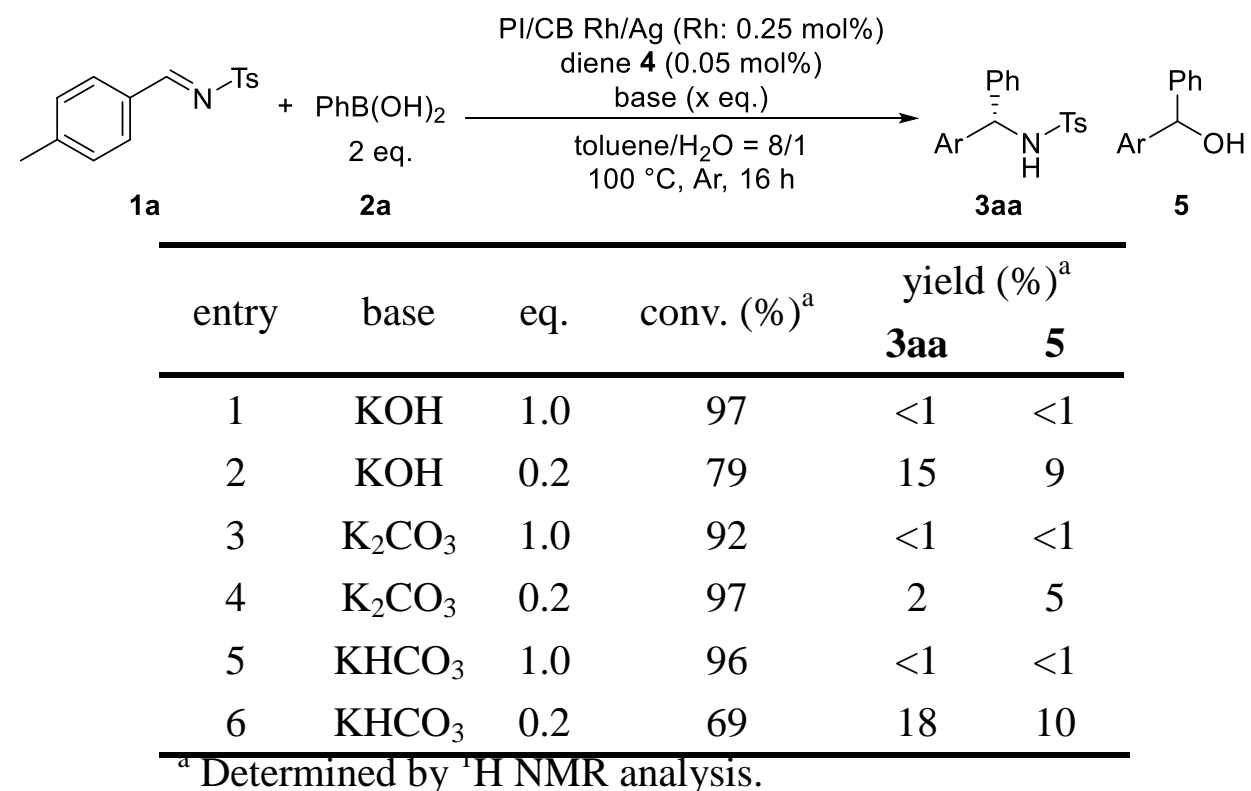

Table S2. Screening of metal triflates as Lewis acid.

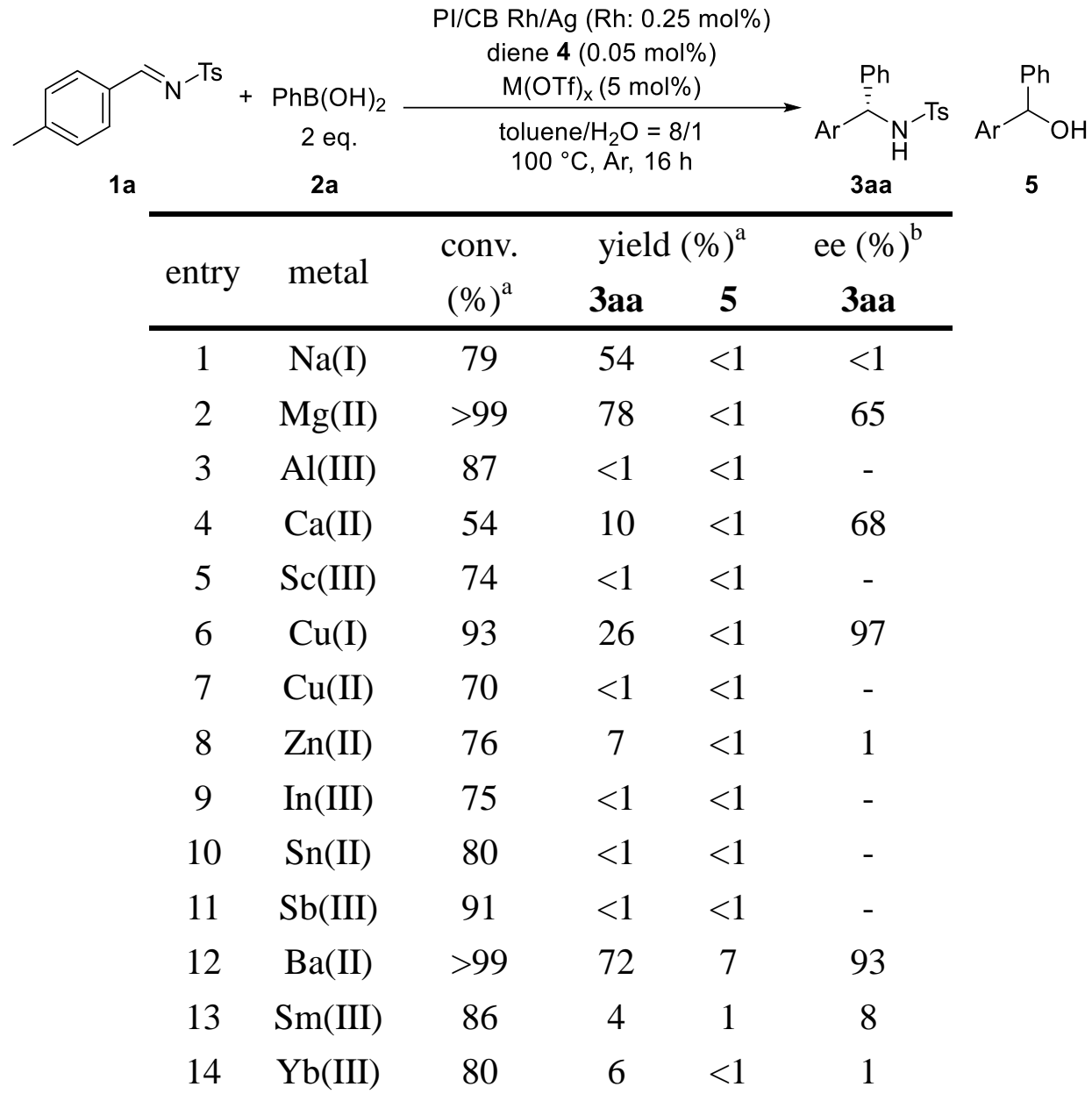




\begin{tabular}{llllll}
15 & $\mathrm{Hf}(\mathrm{IV})$ & 52 & $<1$ & $<1$ & - \\
16 & $\mathrm{Bi}(\mathrm{III})$ & 81 & $<1$ & $<1$ & - \\
\hline${ }^{\mathrm{a}}$ Determined by ${ }^{1} \mathrm{H}$ NMR analysis. & ${ }^{\mathrm{b}}$ Determined by \\
HPLC analysis.
\end{tabular}

\section{References}

[1] Chen, F.-X.; Kina, A.; Hayashi, T. Org. Lett. 2006, 8, 341.

[2] Chemla, F.; Hebbe, V.; Normant, J. F. Synthesis (Stuttg). 2000, 75-77

[3] T. Yasukawa, A. Suzuki, H. Miyamura, K. Nishino, S. Kobayashi, J. Am. Chem. Soc., 2015, 137, 6616.

[4] T. Yasukawa, H. Miyamura, S. Kobayashi, J. Am. Chem. Soc., 2012, 134, 16963.

[5] Wang, Z.; Feng, C.; Xu, M.; Lin, G. J. Am. Chem. Soc. 2007, 129, 5336-5337.

[6] Beisel, T.; Manolikakes, G. Org. Lett. 2015, 17, 3162-3165.

[7] Duan, H.-F.; Jia, Y.-X.; Wang, L.-X.; Zhou, Q.-L. Org. Lett. 2006, 8, 25672569.

[8] Chen, C.-C.; Gopula, B.; Syu, J.-F.; Pan, J.-H.; Kuo, T.-S.; Wu, P.-Y.; Henschke, J. P.; Wu, H.-L. J. Org. Chem. 2014, 79, 8077-8085. 


\section{HPLC and NMR charts}<smiles>Cc1ccc(C(NC(F)(F)F)c2ccccc2)cc1</smiles>

$\mathrm{mV}$

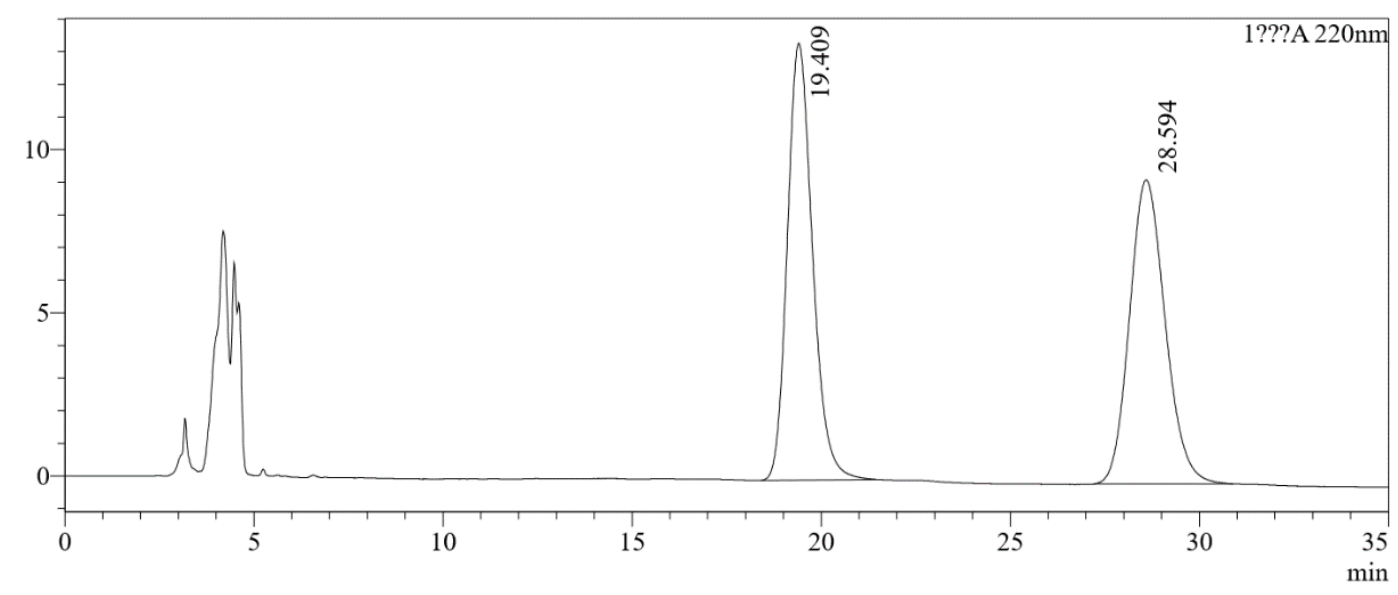

???A 220nm

???A $220 \mathrm{~nm}$
\begin{tabular}{|r|r|r|r|r|}
\hline Peak\# & Ret. Time & \multicolumn{1}{|c|}{ Area } & Height & \multicolumn{1}{|c|}{ Area $\%$} \\
\hline 1 & 19.409 & 620310 & 13389 & 50.394 \\
\hline 2 & 28.594 & 610613 & 9312 & 49.606 \\
\hline
\end{tabular}<smiles>Cc1ccc(C(NC(F)(F)F)c2ccccc2)cc1</smiles>

$\mathrm{mV}$

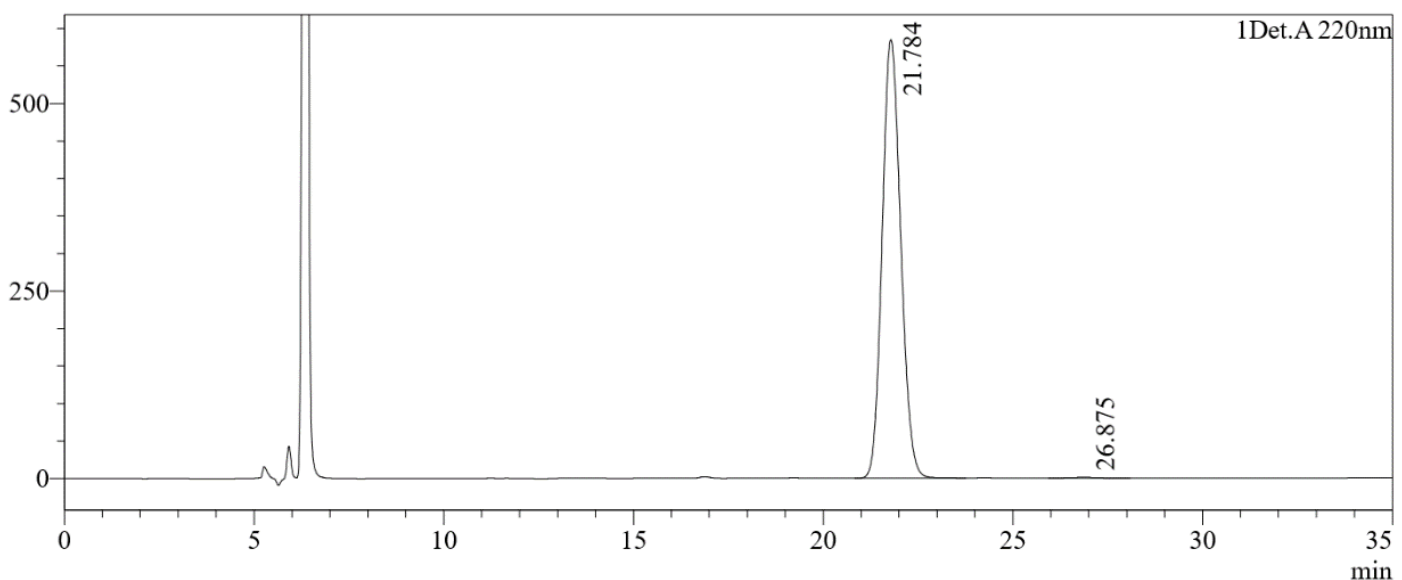

Det.A 220nm

\begin{tabular}{|r|r|r|r|r|}
\hline \multicolumn{1}{|c|}{ Peak\# } & Ret. Time & \multicolumn{1}{c|}{ Area } & Height & \multicolumn{1}{c|}{ Area\% } \\
\hline 1 & 21.784 & 19914111 & 584693 & 99.789 \\
\hline 2 & 26.875 & 42078 & 923 & 0.211 \\
\hline
\end{tabular}




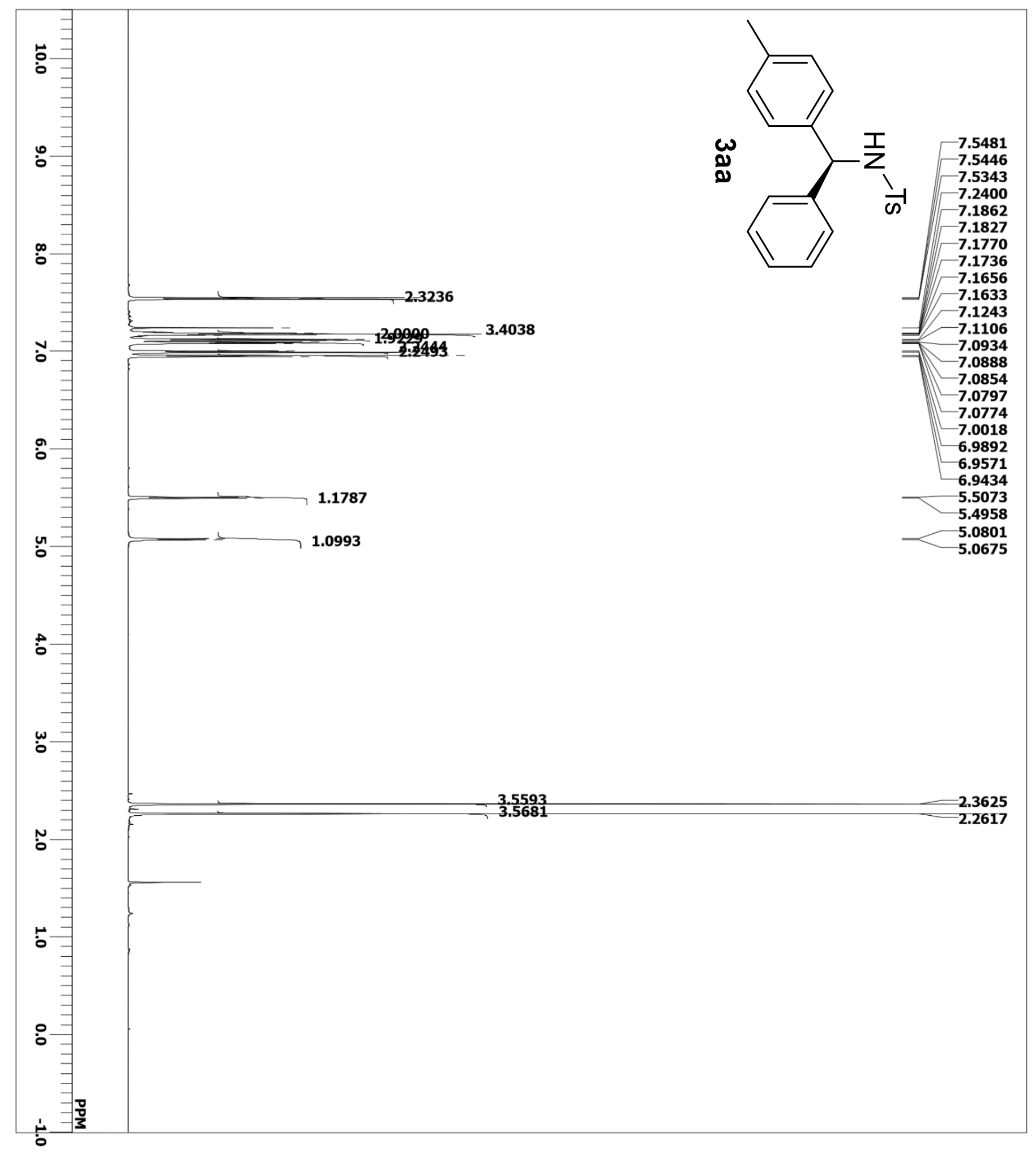




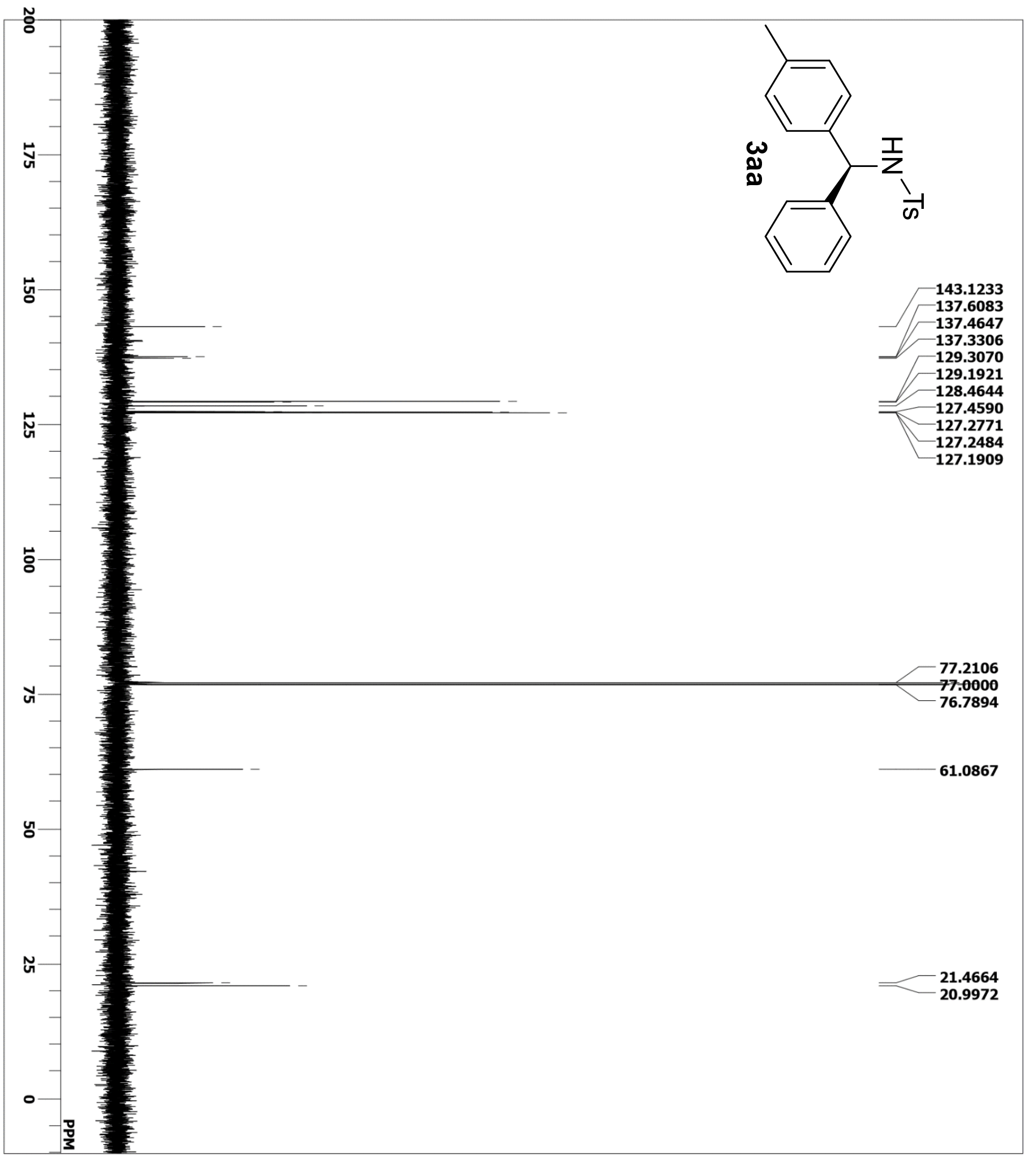




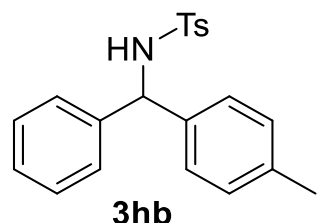

(ent-3aa)

$\mathrm{mV}$
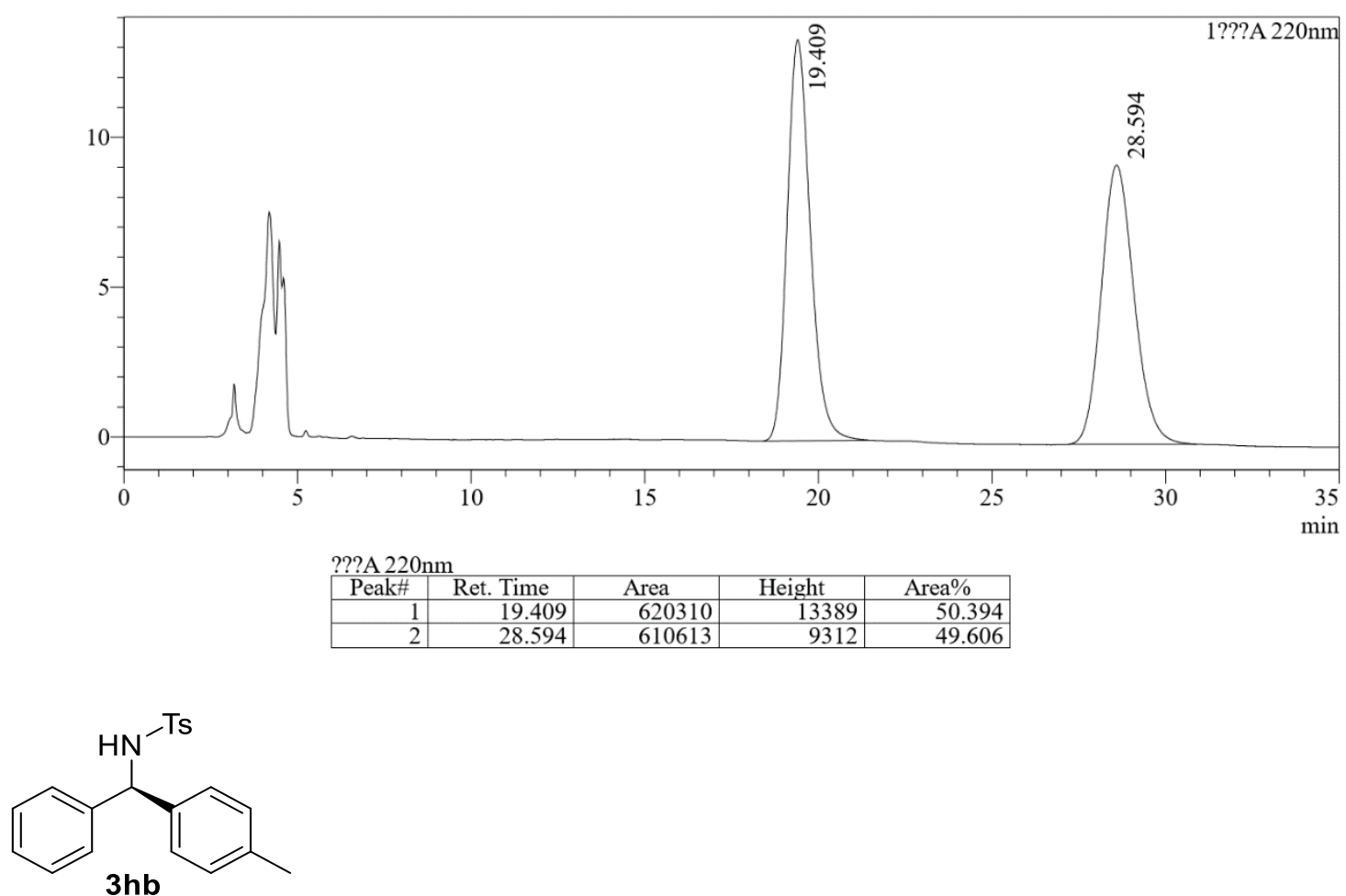

(ent-3aa)

$\mathrm{mV}$

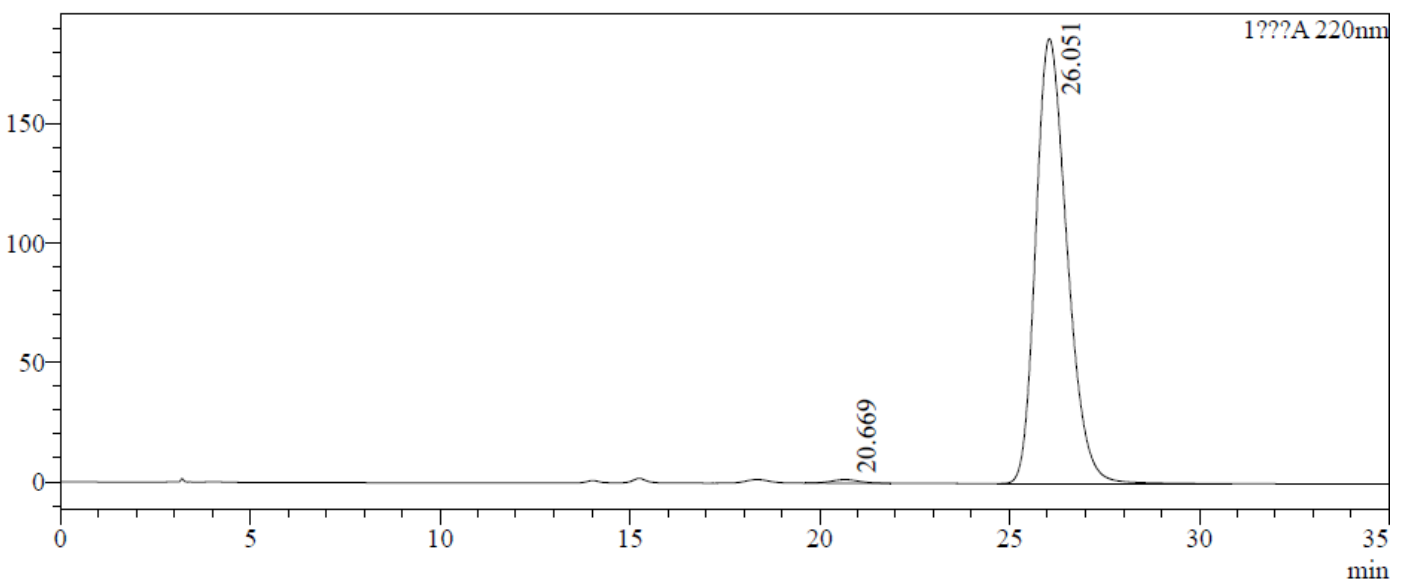

???A $220 \mathrm{~nm}$
\begin{tabular}{|r|r|r|r|r|}
\hline Peak\# & Ret. Time & Area & Height & Area $\%$ \\
\hline 1 & 20.669 & 80187 & 1594 & 0.743 \\
\hline 2 & 26.051 & 10704919 & 186378 & 99.257 \\
\hline
\end{tabular}


<smiles>Cc1cccc(C(NC(F)(F)F)c2ccccc2)c1</smiles>

$\mathrm{mV}$

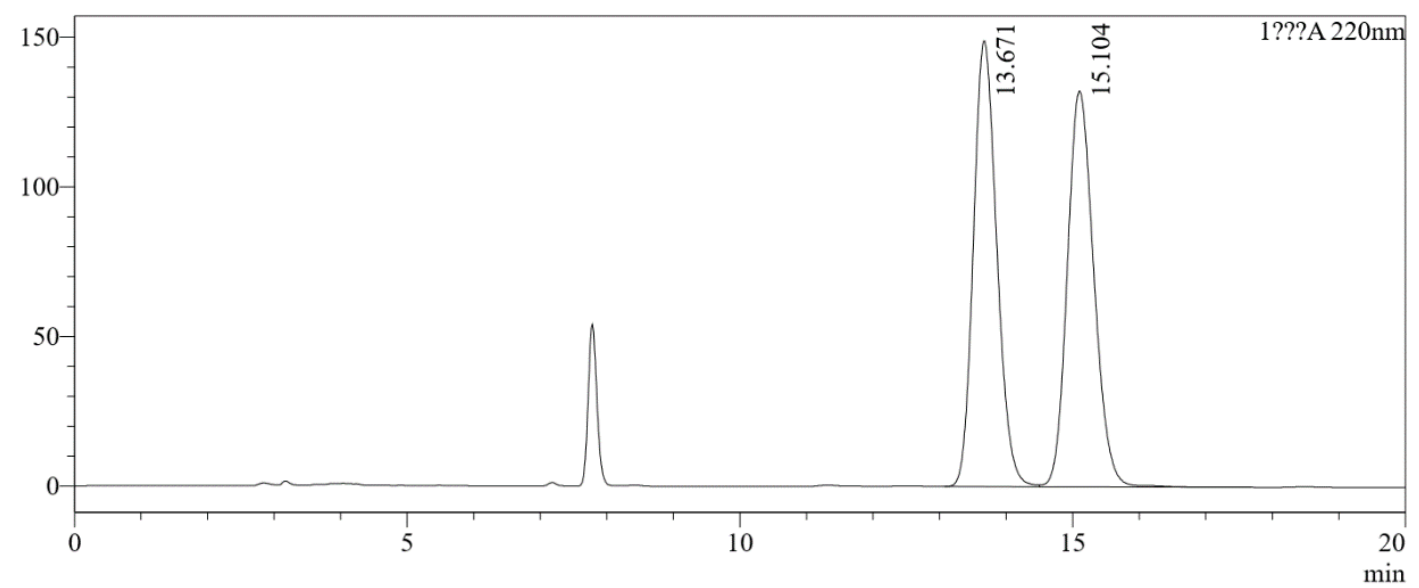

???A $220 \mathrm{~nm}$
\begin{tabular}{|r|r|r|r|r|}
\hline Peak\# & Ret. Time & Area & Height & \multicolumn{1}{|c|}{ Area $\%$} \\
\hline 1 & 13.671 & 3636575 & 149019 & 49.786 \\
\hline 2 & 15.104 & 3667856 & 132224 & 50.214 \\
\hline
\end{tabular}<smiles>Cc1cccc(C(NC(F)(F)F)c2ccccc2)c1</smiles>

$\mathrm{mV}$

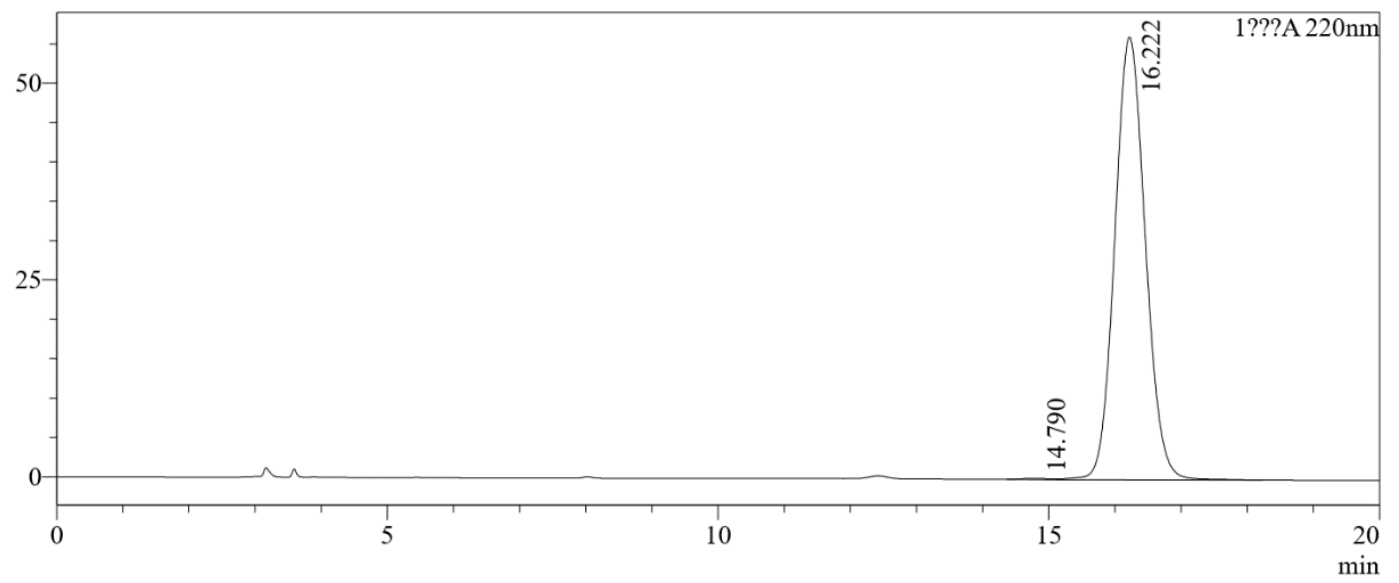

???A 220nm
\begin{tabular}{|r|r|r|r|r|}
\hline Peak\# & Ret. Time & \multicolumn{1}{|c|}{ Area } & Height & \multicolumn{1}{|c|}{ Area $\%$} \\
\hline 1 & 14.790 & 3854 & 138 & 0.213 \\
\hline 2 & 16.222 & 1805655 & 56225 & 99.787 \\
\hline
\end{tabular}




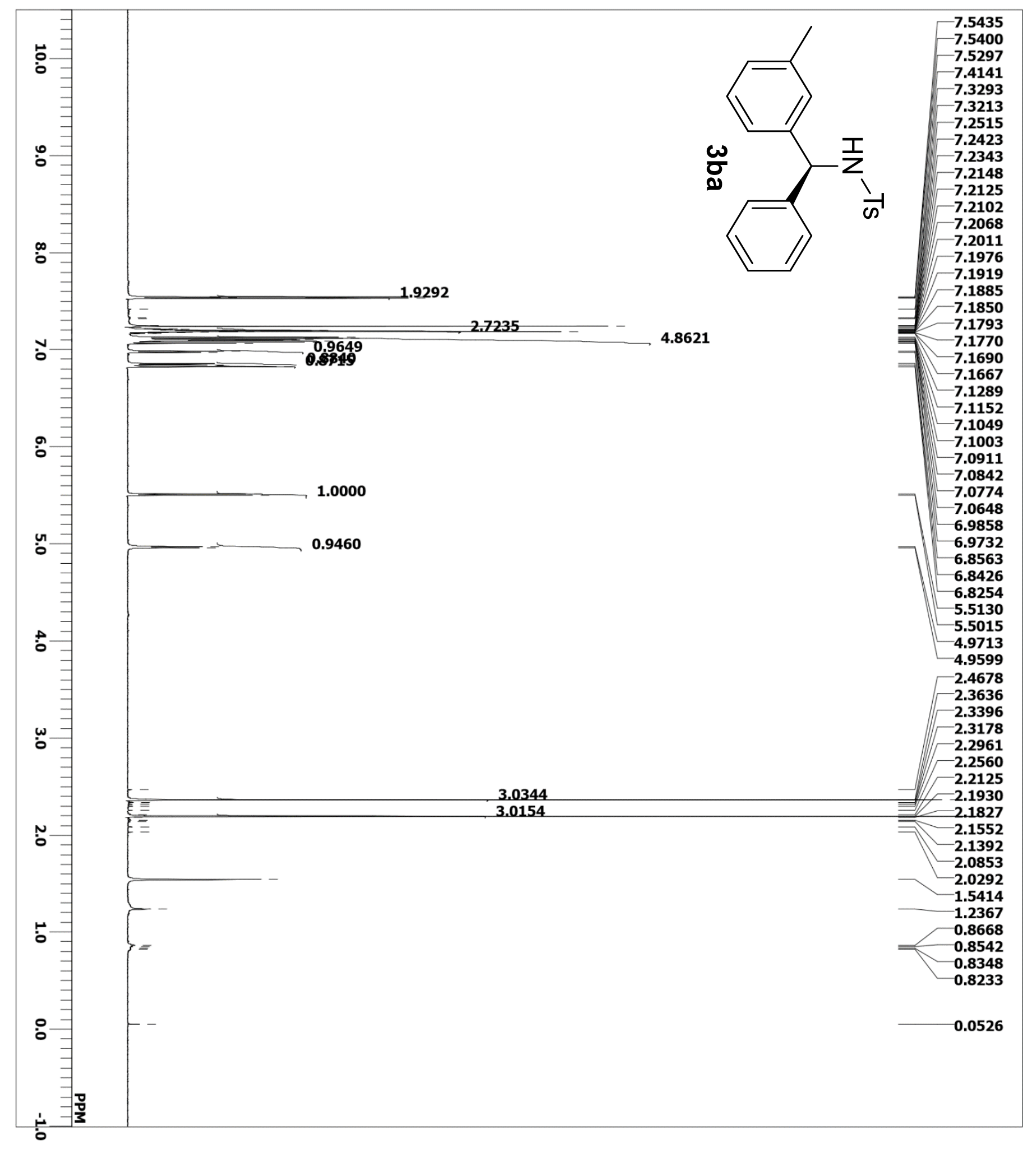




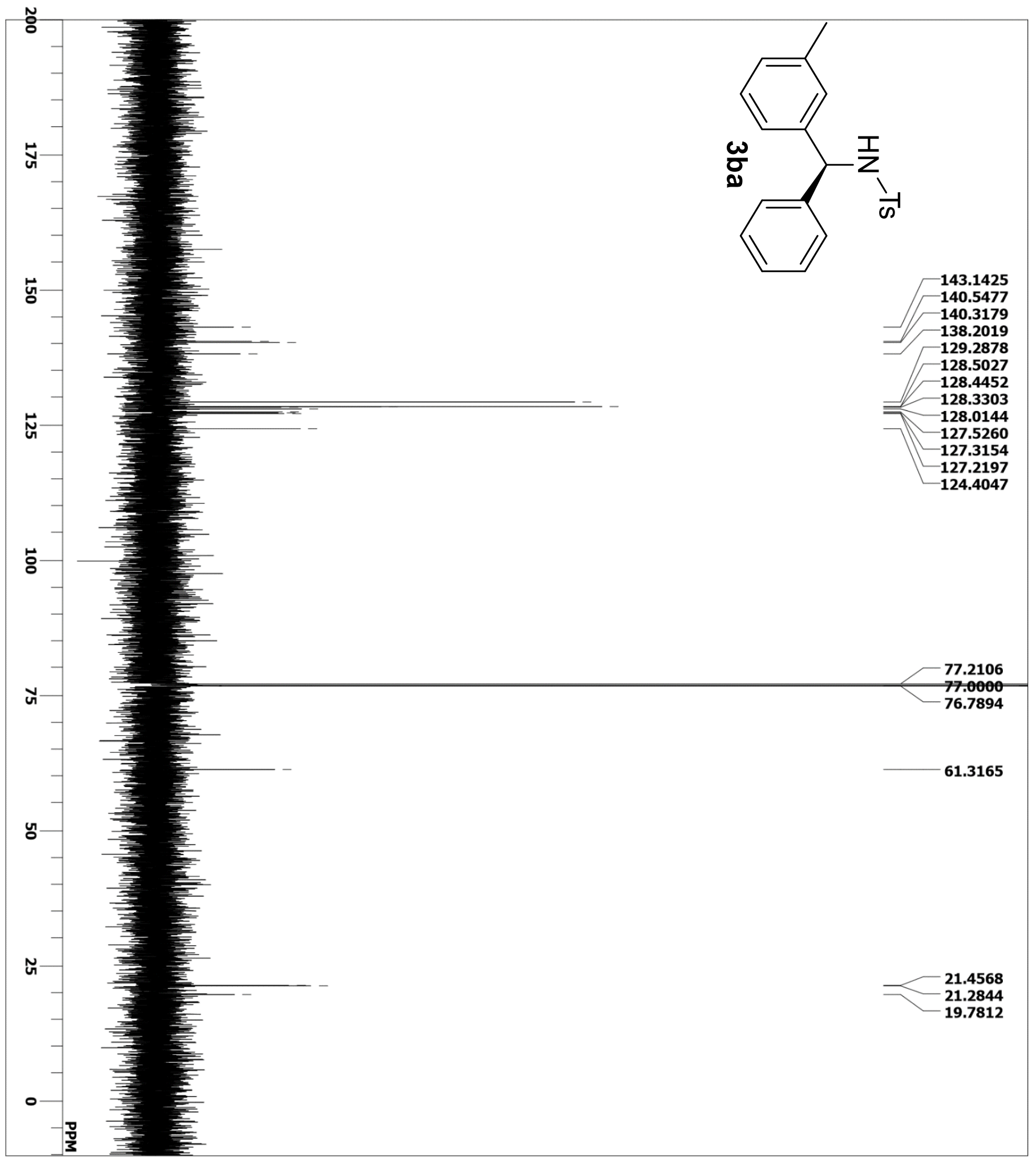


<smiles>Cc1ccccc1C(NC(F)(F)F)c1ccccc1</smiles>

$3 \mathrm{ca}$

$\mathrm{mV}$

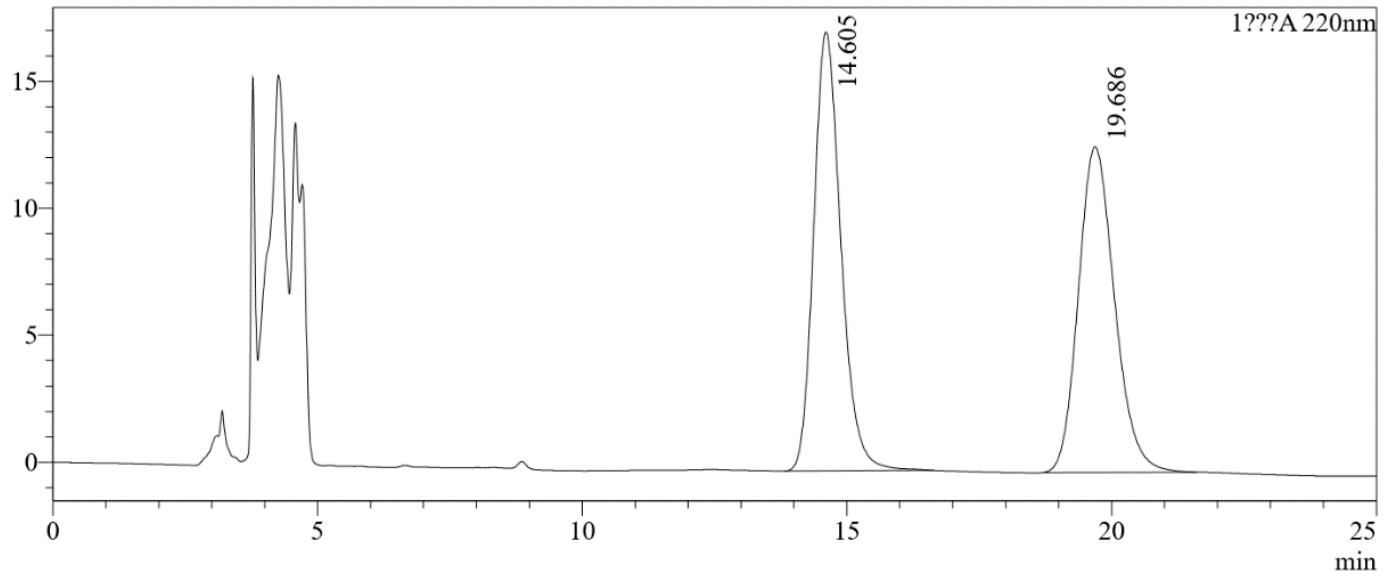

???A 220nm

\begin{tabular}{|r|r|r|r|r|}
\hline Peak\# & Ret. Time & \multicolumn{1}{|c|}{ Area } & Height & \multicolumn{1}{|c|}{ Area\% } \\
\hline 1 & 14.605 & 618338 & 17272 & 50.275 \\
\hline 2 & 19.686 & 611574 & 12831 & 49.725 \\
\hline
\end{tabular}<smiles>Cc1ccccc1[C@H](NC(F)(F)F)c1ccccc1</smiles>

$3 \mathrm{ca}$

$\mathrm{mV}$

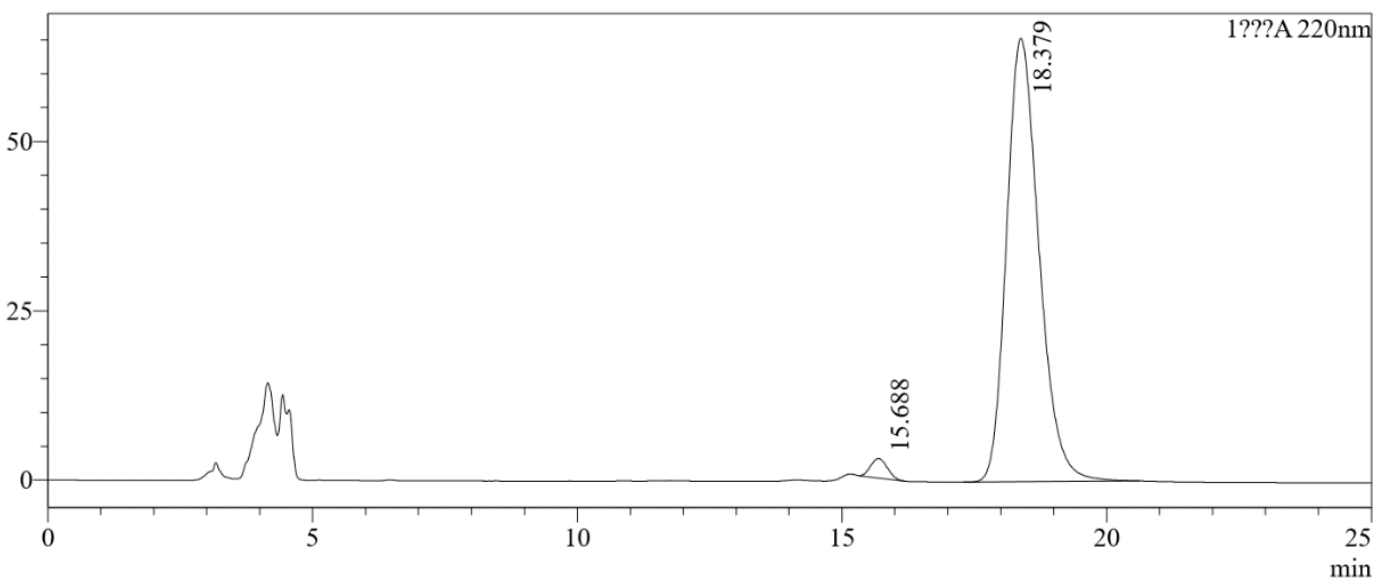

???A220nm
\begin{tabular}{|r|r|r|r|r|}
\hline Peak\#t & Ret. Time & Area & Height & \multicolumn{1}{c|}{ Area\% } \\
\hline 1 & 15.688 & 62663 & 2858 & 2.195 \\
\hline 2 & 18.379 & 2792286 & 65444 & 97.805 \\
\hline
\end{tabular}




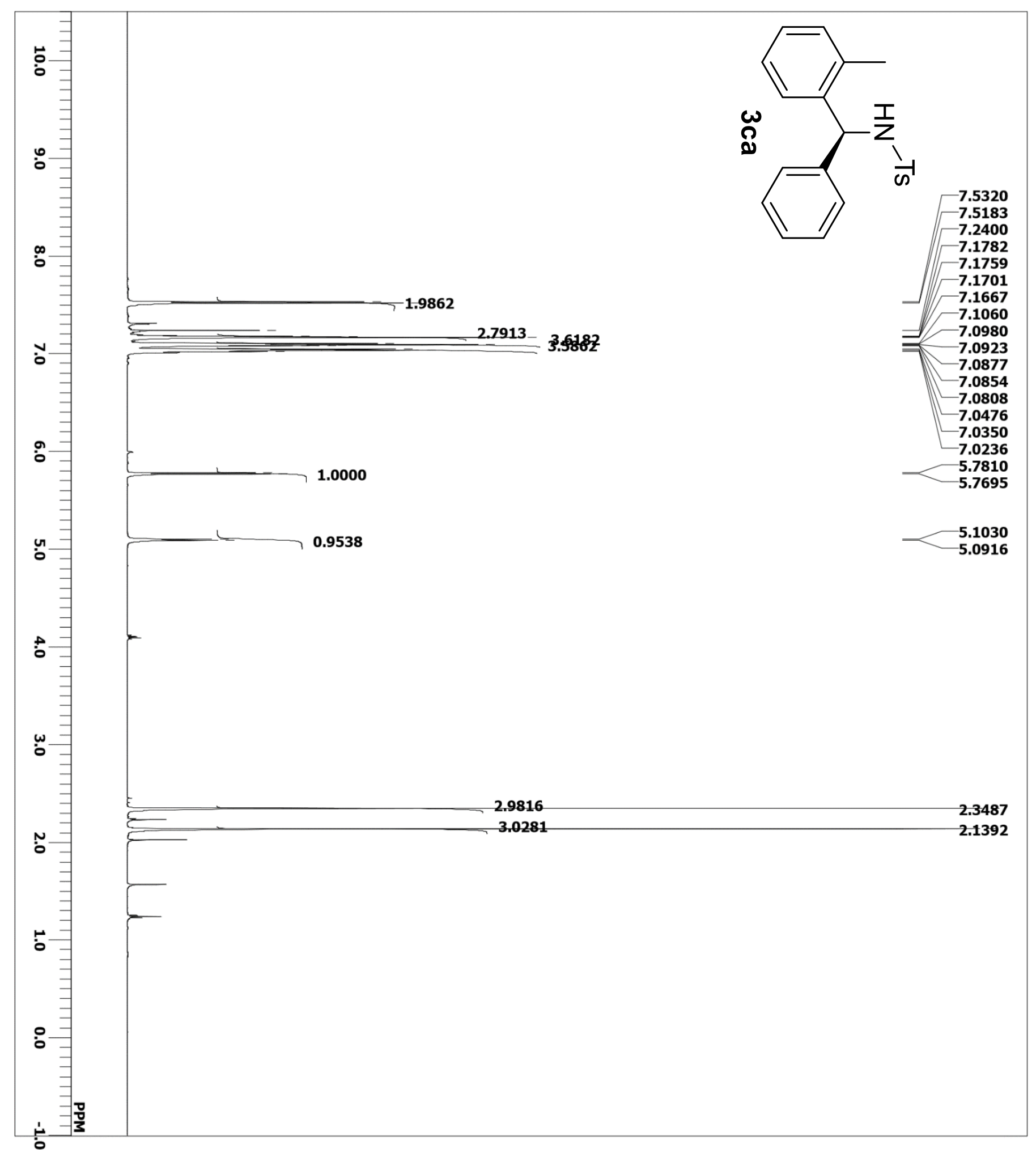




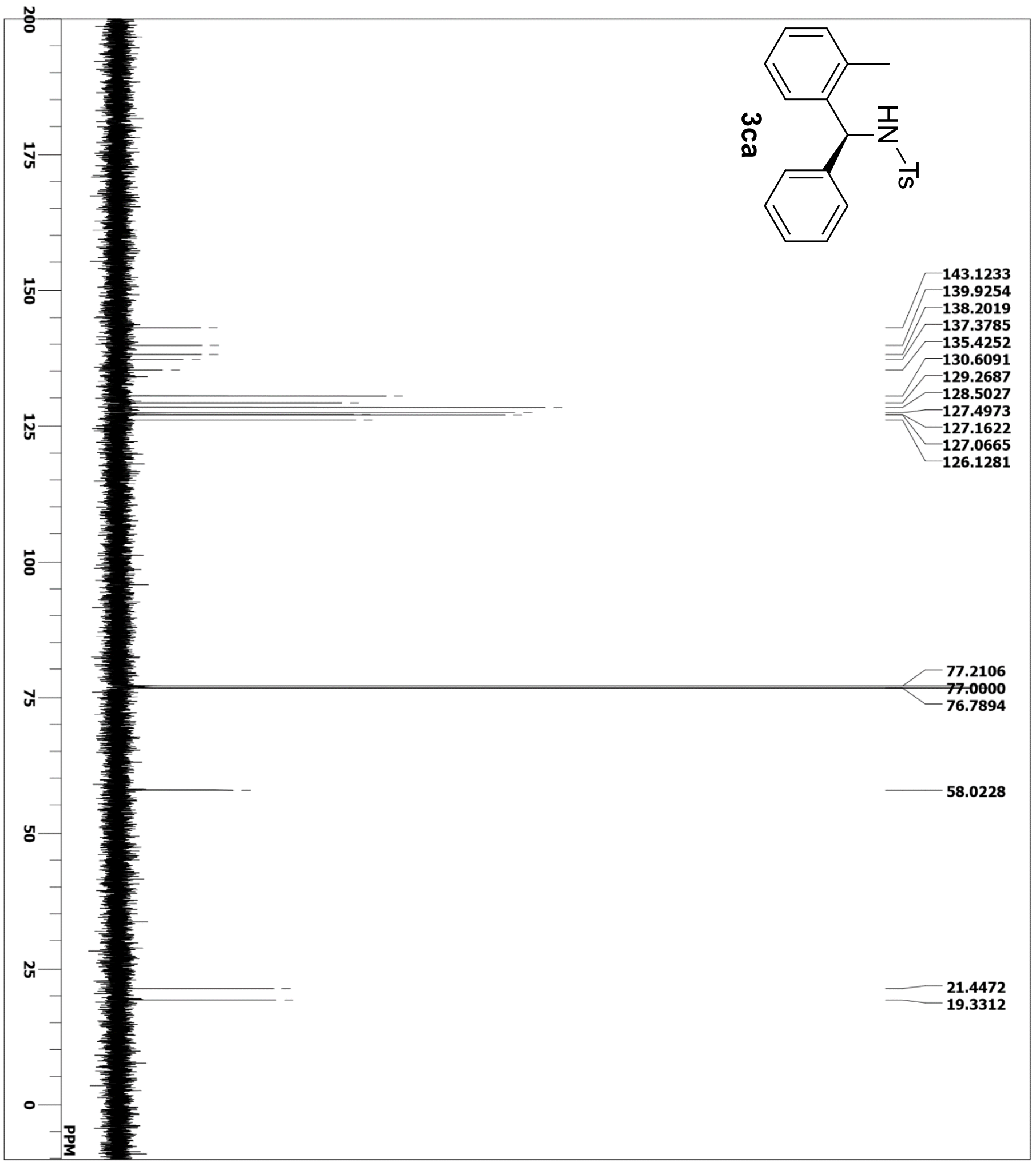


<smiles>Fc1ccc(C(NC(F)(F)F)c2ccccc2)cc1</smiles>

$\mathrm{mV}$

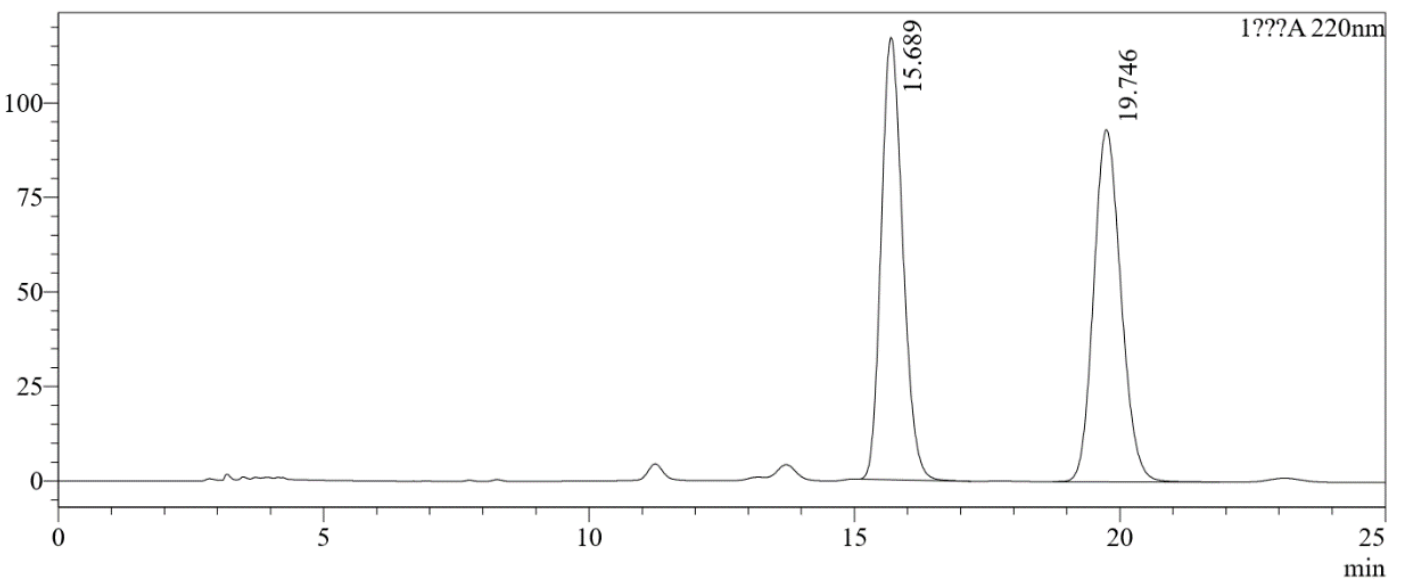

???A 220nm

\begin{tabular}{|r|r|r|r|r|}
\hline Peak\# & Ret. Time & Area & \multicolumn{1}{c|}{ Height } & \multicolumn{1}{|c|}{ Area\% } \\
\hline 1 & 15.689 & 3296900 & 116963 & 49.754 \\
\hline 2 & 19.746 & 3329525 & 93121 & 50.246 \\
\hline
\end{tabular}

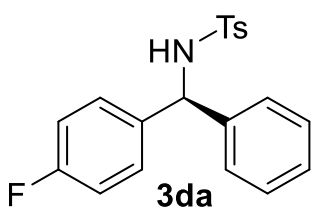

$\mathrm{mV}$

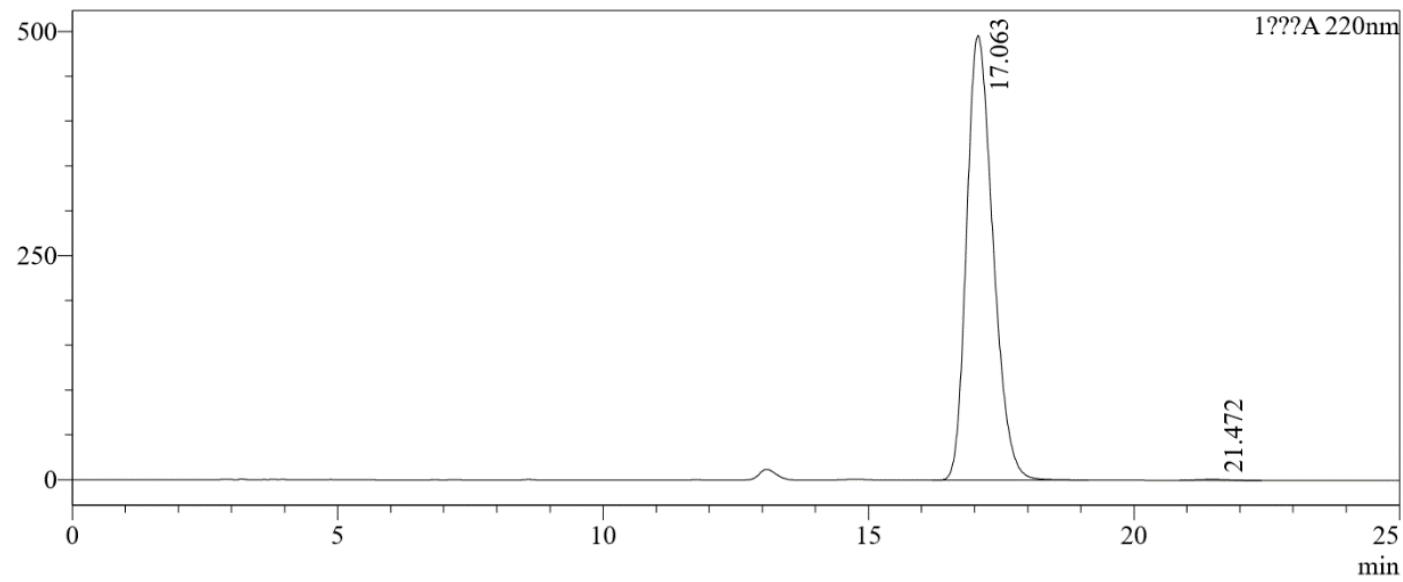

???A 220nm
\begin{tabular}{|r|r|r|r|r|}
\hline Peak\# & Ret. Time & \multicolumn{1}{c|}{ Area } & Height & \multicolumn{1}{|c|}{ Area $\%$} \\
\hline 1 & 17.063 & 17180134 & 496068 & 99.749 \\
\hline 2 & 21.472 & 43145 & 1072 & 0.251 \\
\hline
\end{tabular}




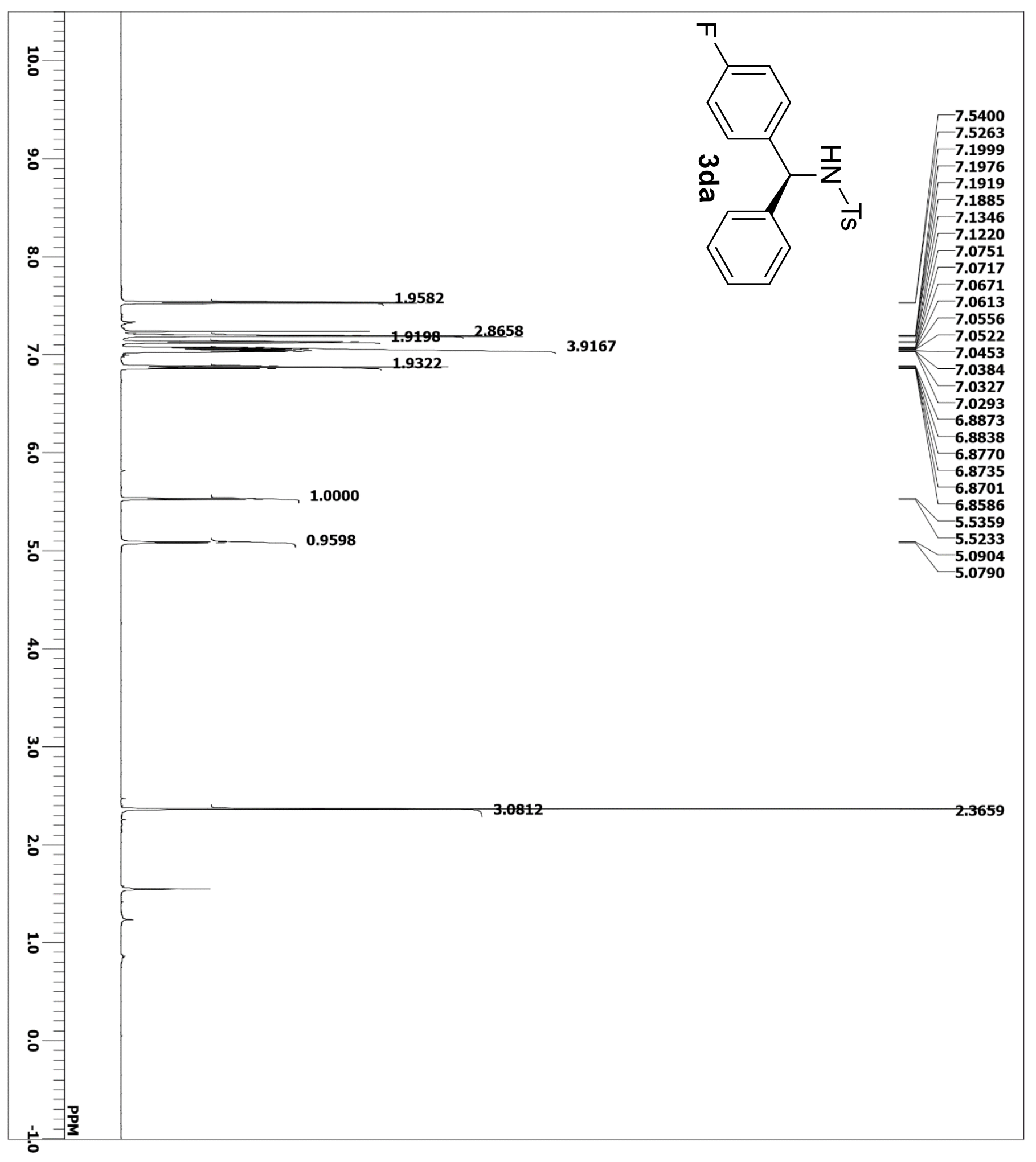




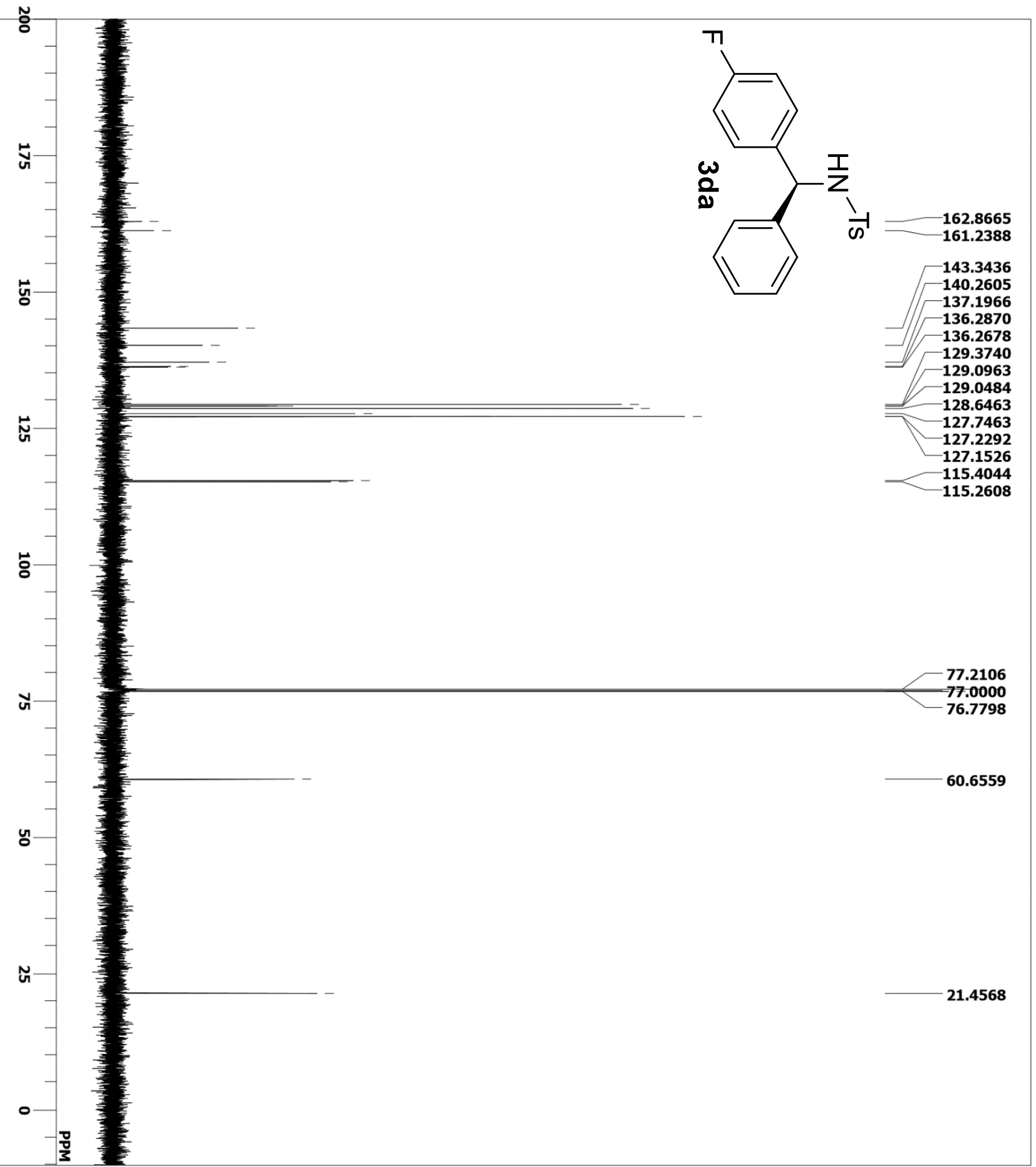


<smiles>FNC(c1ccccc1)c1ccc(Cl)cc1</smiles>

$\mathrm{mV}$

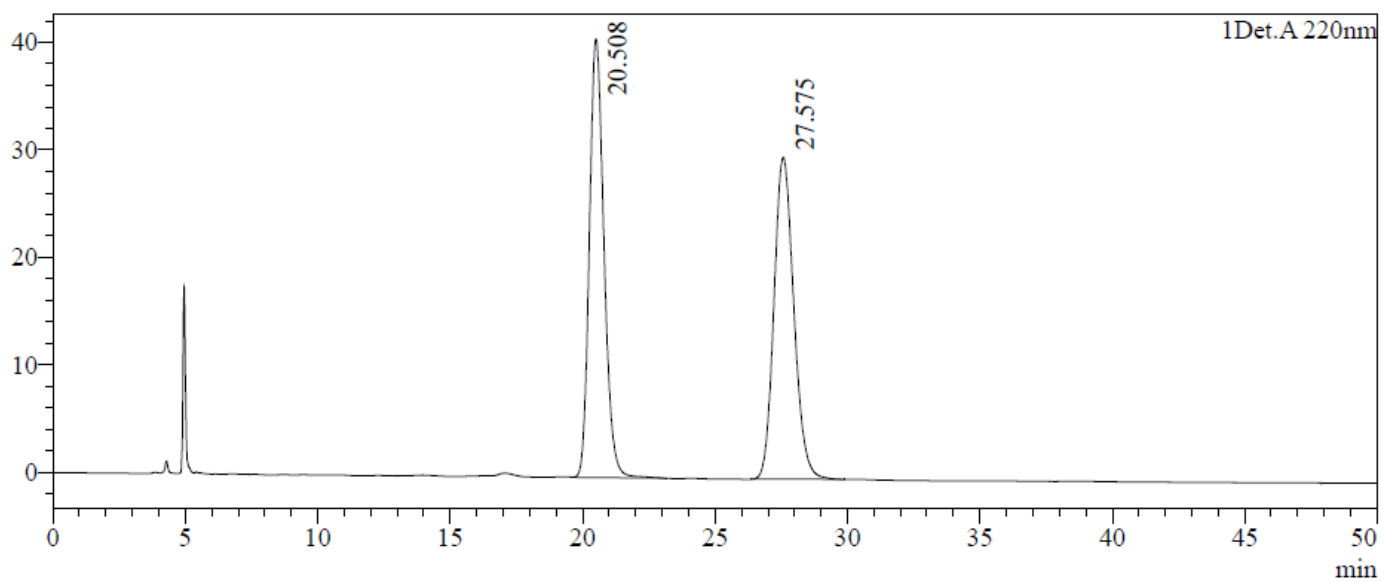

Det.A 220nm

\begin{tabular}{r|r|r|r|r|}
\hline \multicolumn{1}{|c|}{ Peak\# } & Ret. Time & Area & Height & \multicolumn{1}{|c|}{ Area\% } \\
\hline 1 & 20.508 & 1560802 & 40774 & 50.080 \\
\hline 2 & 27.575 & 1555784 & 29951 & 49.920 \\
\hline
\end{tabular}

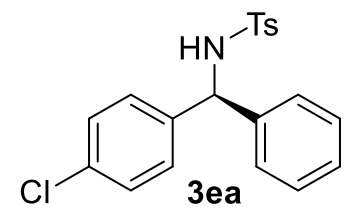

$\mathrm{mV}$

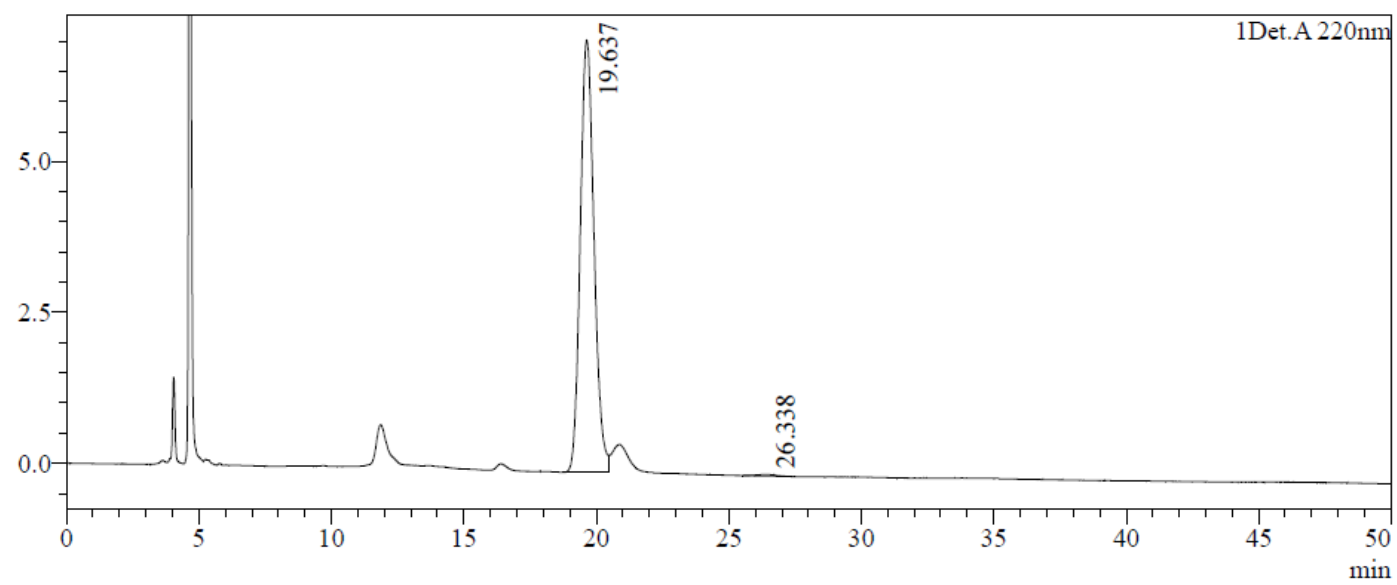

Det.A $220 \mathrm{~nm}$
\begin{tabular}{|r|r|r|r|r|}
\hline Peak\# & Ret. Time & Area & Height & \multicolumn{1}{|c|}{ Area $\%$} \\
\hline 1 & 19.637 & 259497 & 7173 & 99.518 \\
\hline 2 & 26.338 & 1256 & 26 & 0.482 \\
\hline
\end{tabular}




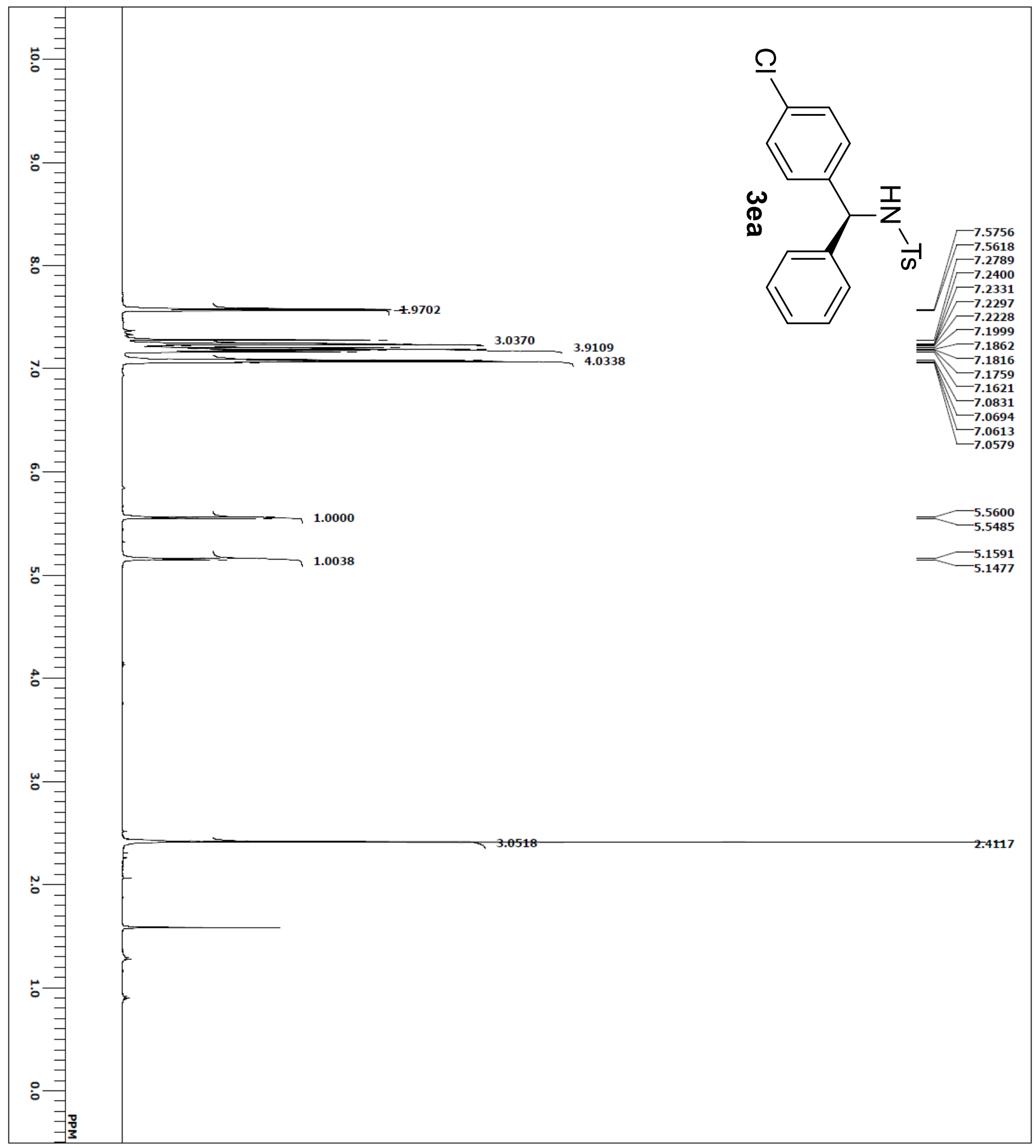




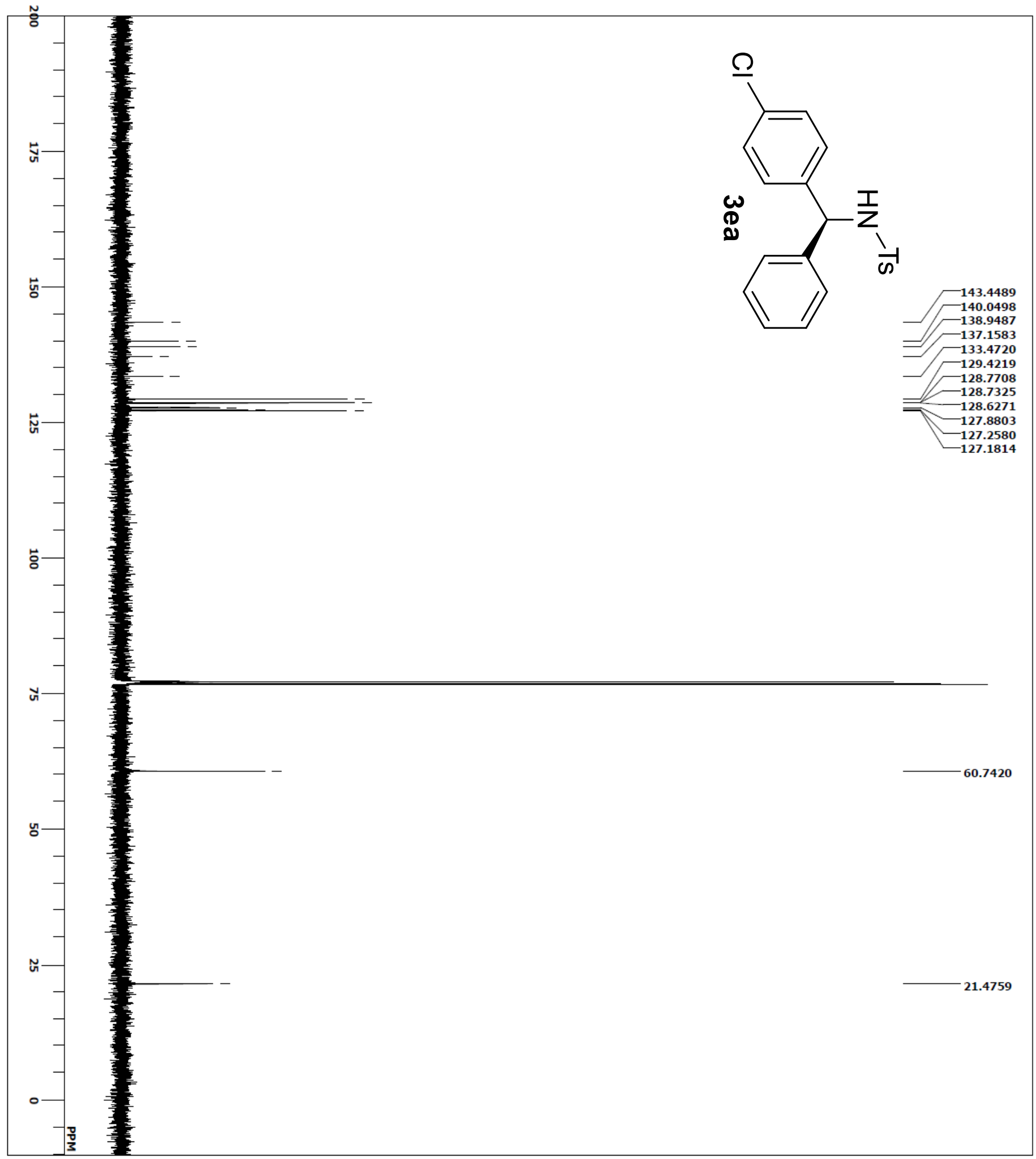


<smiles>FC(F)(F)NC(c1ccccc1)c1ccc(Br)cc1</smiles>

$\mathrm{mV}$
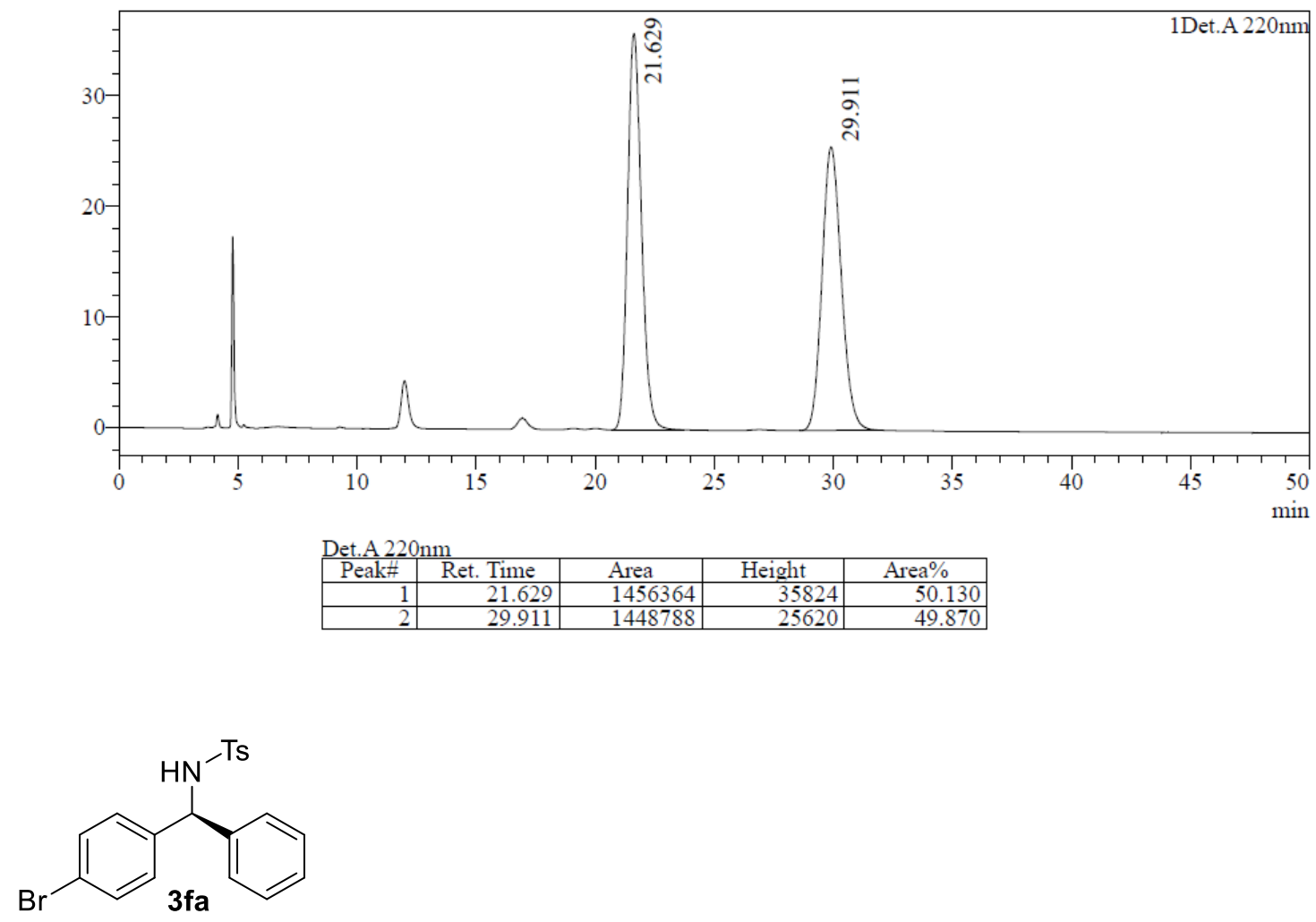

$\mathrm{mV}$

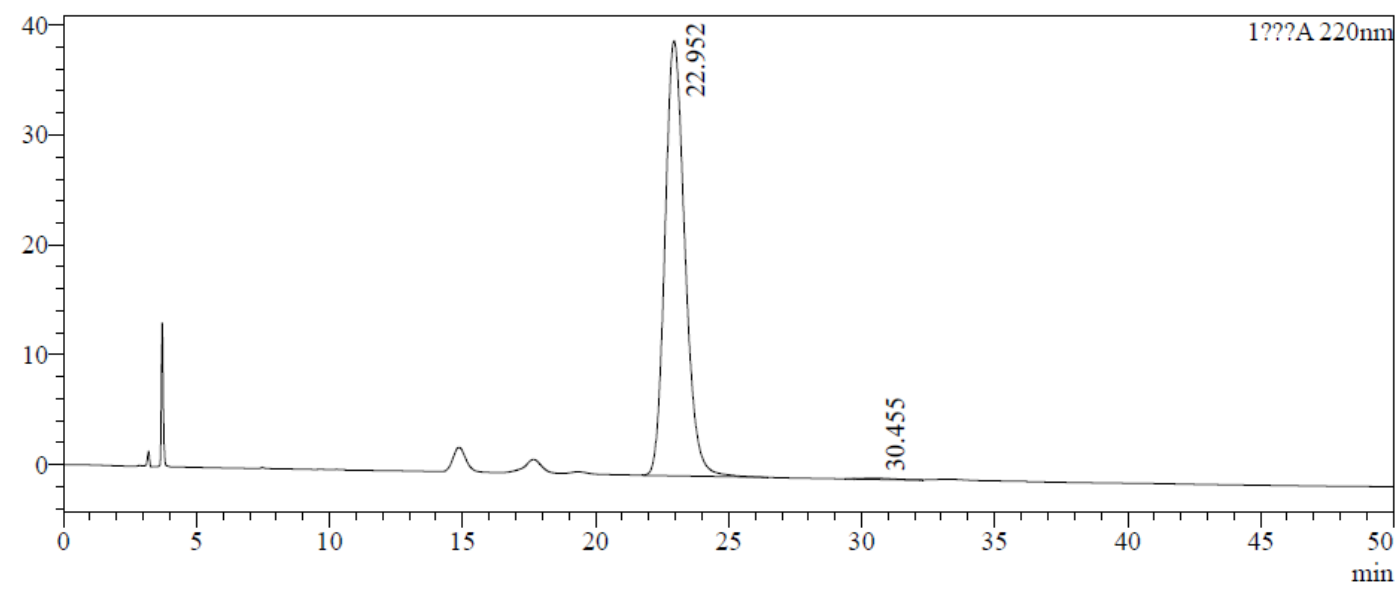

???A $220 \mathrm{~nm}$
\begin{tabular}{|r|r|r|r|r|}
\hline Peak\# & Ret. Time & \multicolumn{1}{|c|}{ Area } & Height & \multicolumn{1}{c|}{ Area $\%$} \\
\hline 1 & 22.952 & 2067219 & 39599 & 99.637 \\
\hline 2 & 30.455 & 7523 & 100 & 0.363 \\
\hline
\end{tabular}




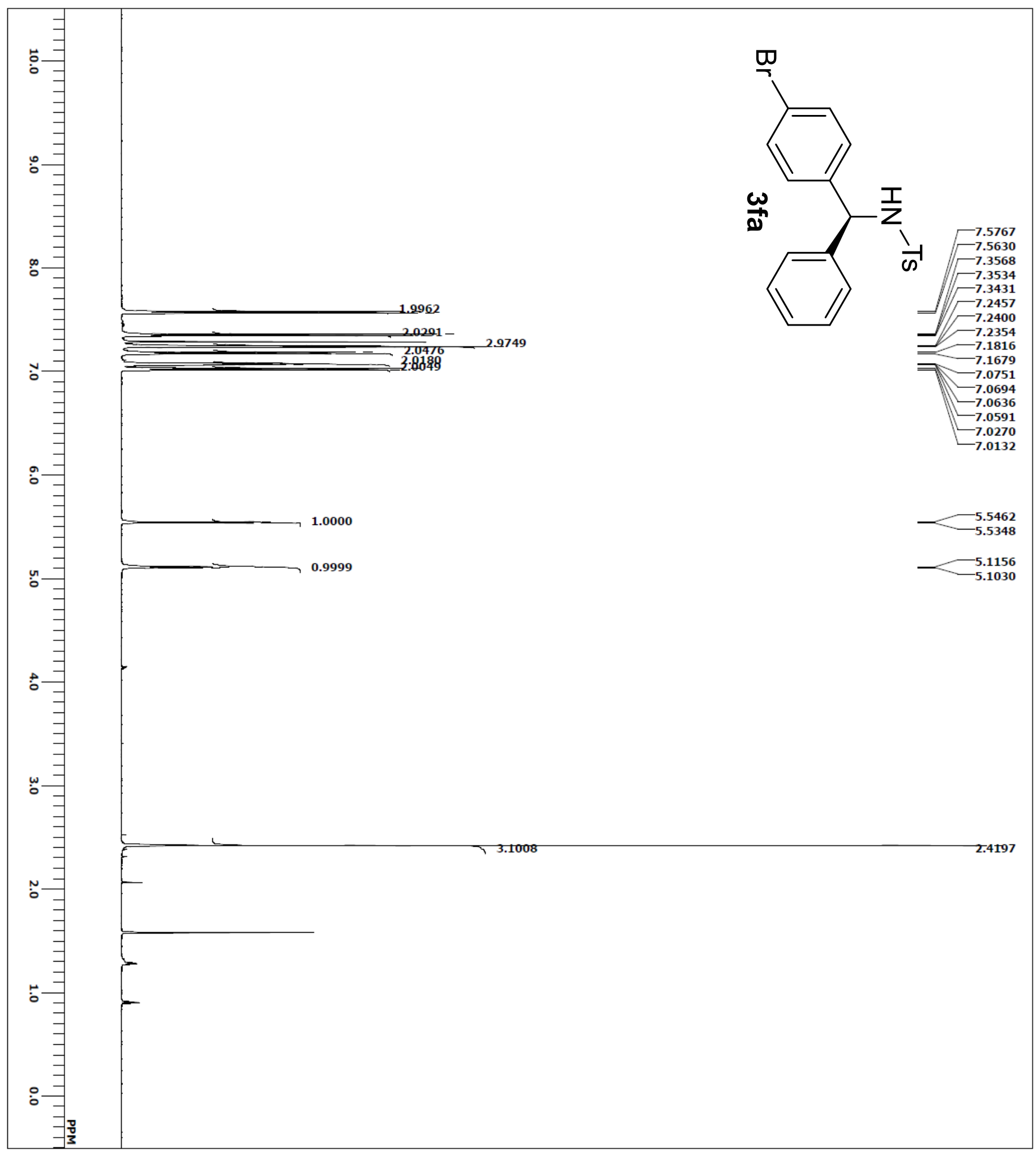




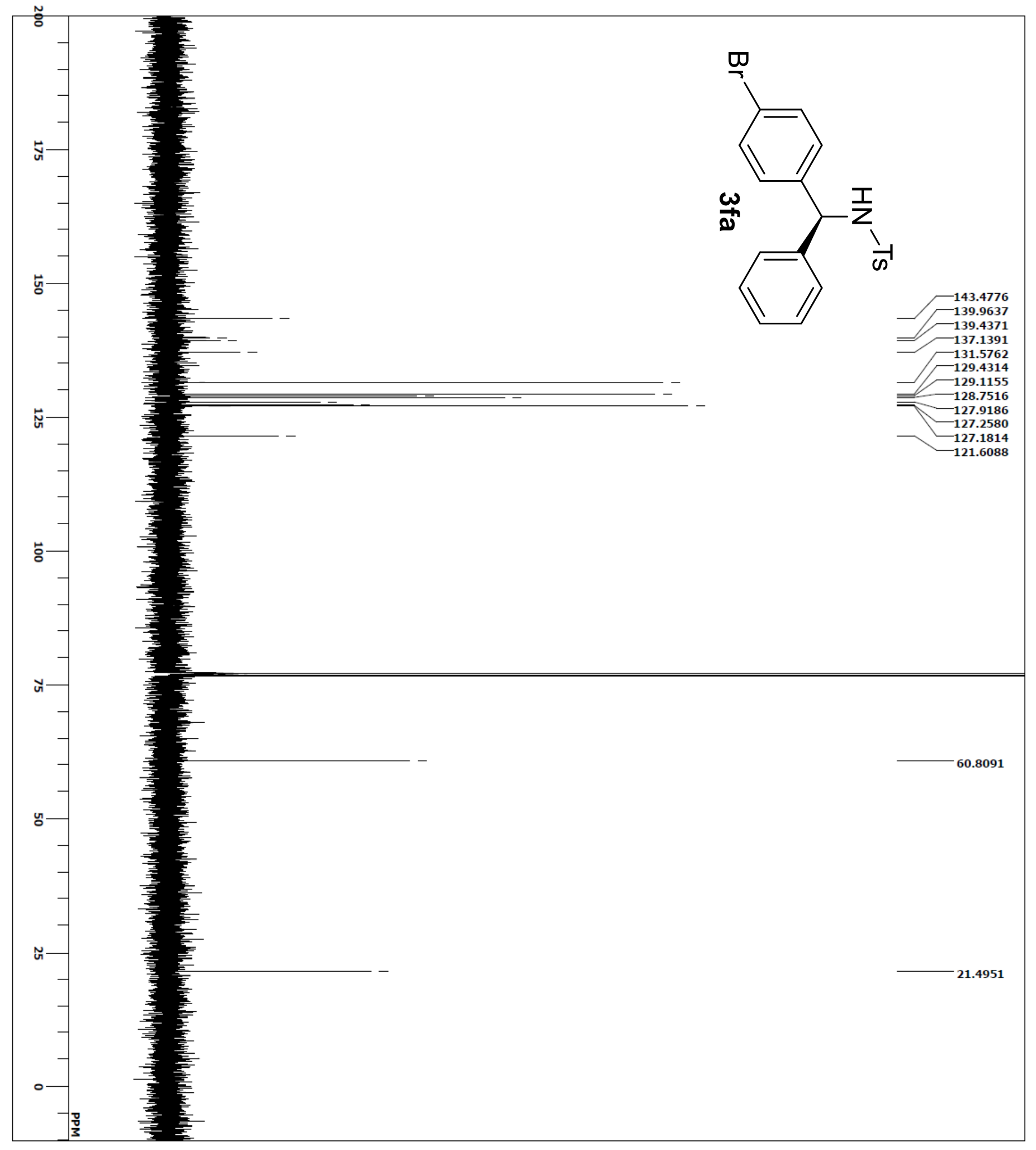


<smiles>COc1ccc(C(NC(F)(F)F)c2ccccc2)cc1</smiles>

$\mathrm{mV}$

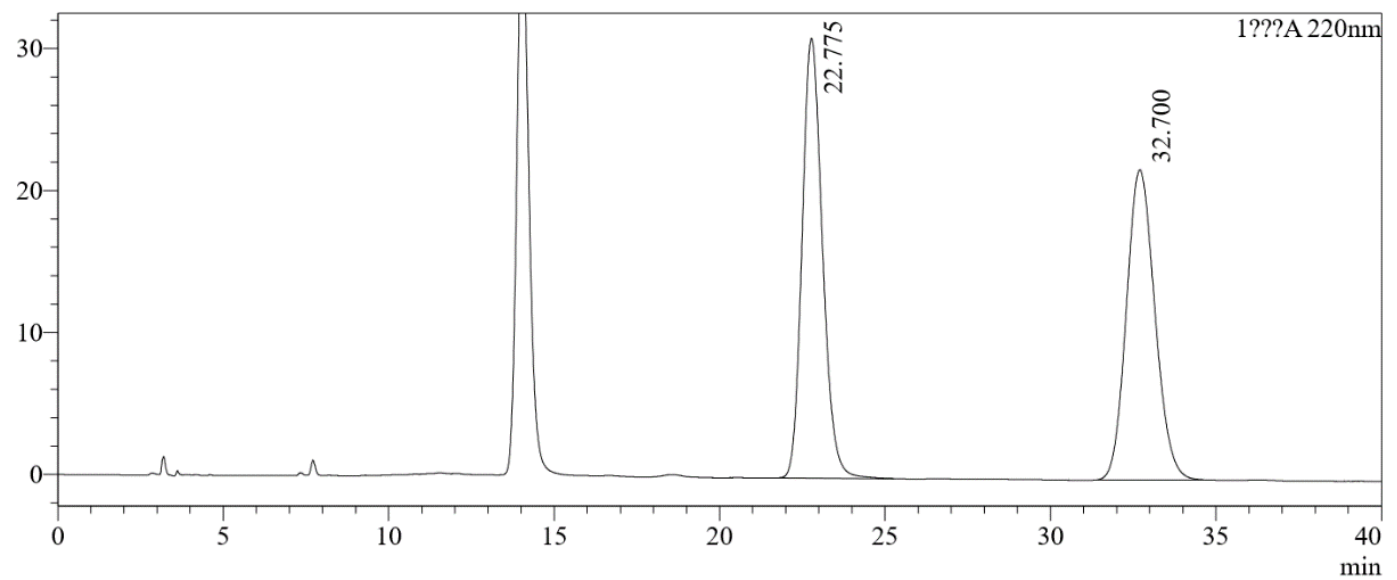

???A $220 \mathrm{~nm}$
\begin{tabular}{|r|r|r|r|r|}
\hline Peak\# & Ret. Time & Area & Height & \multicolumn{1}{|c|}{ Area $\%$} \\
\hline 1 & 22.775 & 1322049 & 30989 & 50.250 \\
\hline 2 & 32.700 & 1308898 & 21853 & 49.750 \\
\hline
\end{tabular}

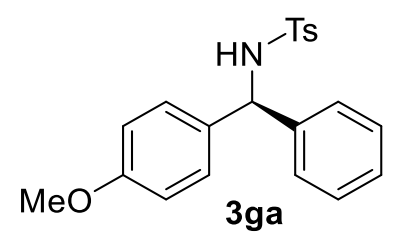

$\mathrm{mV}$

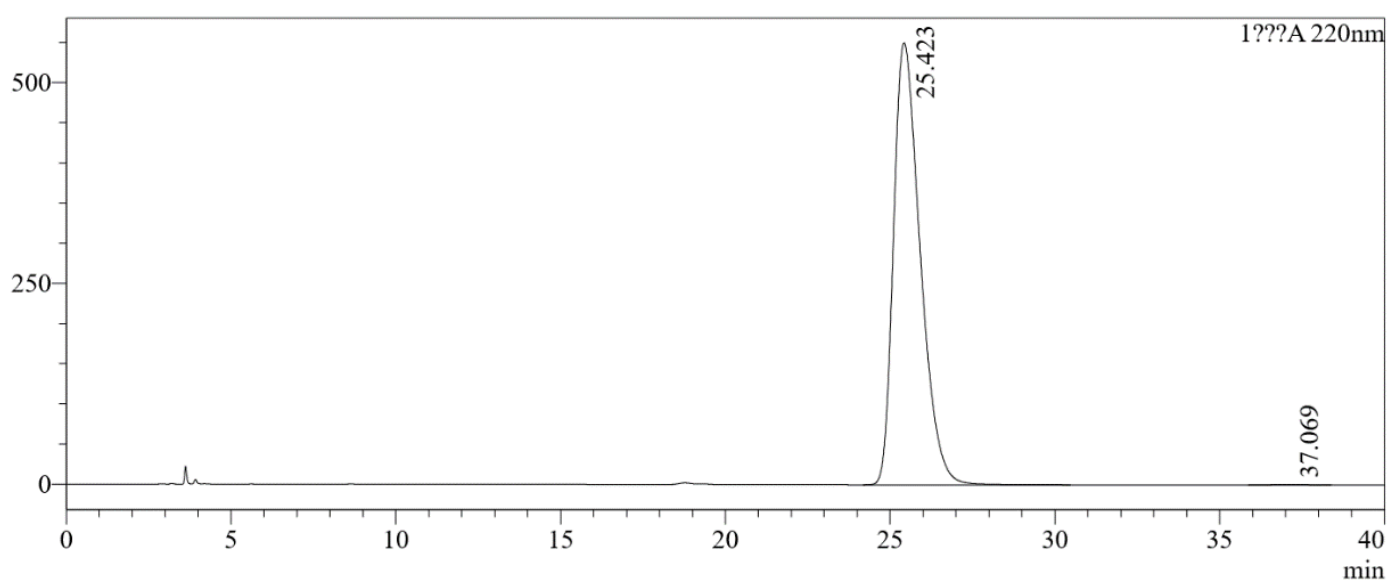

???A $220 \mathrm{~nm}$
\begin{tabular}{|r|r|r|r|r|}
\hline Peak\# & Ret. Time & \multicolumn{1}{c|}{ Area } & \multicolumn{1}{c|}{ Height } & \multicolumn{1}{c|}{ Area $\%$} \\
\hline 1 & 25.423 & 30999103 & 550410 & 99.882 \\
\hline 2 & 37.069 & 36576 & 507 & 0.118 \\
\hline
\end{tabular}




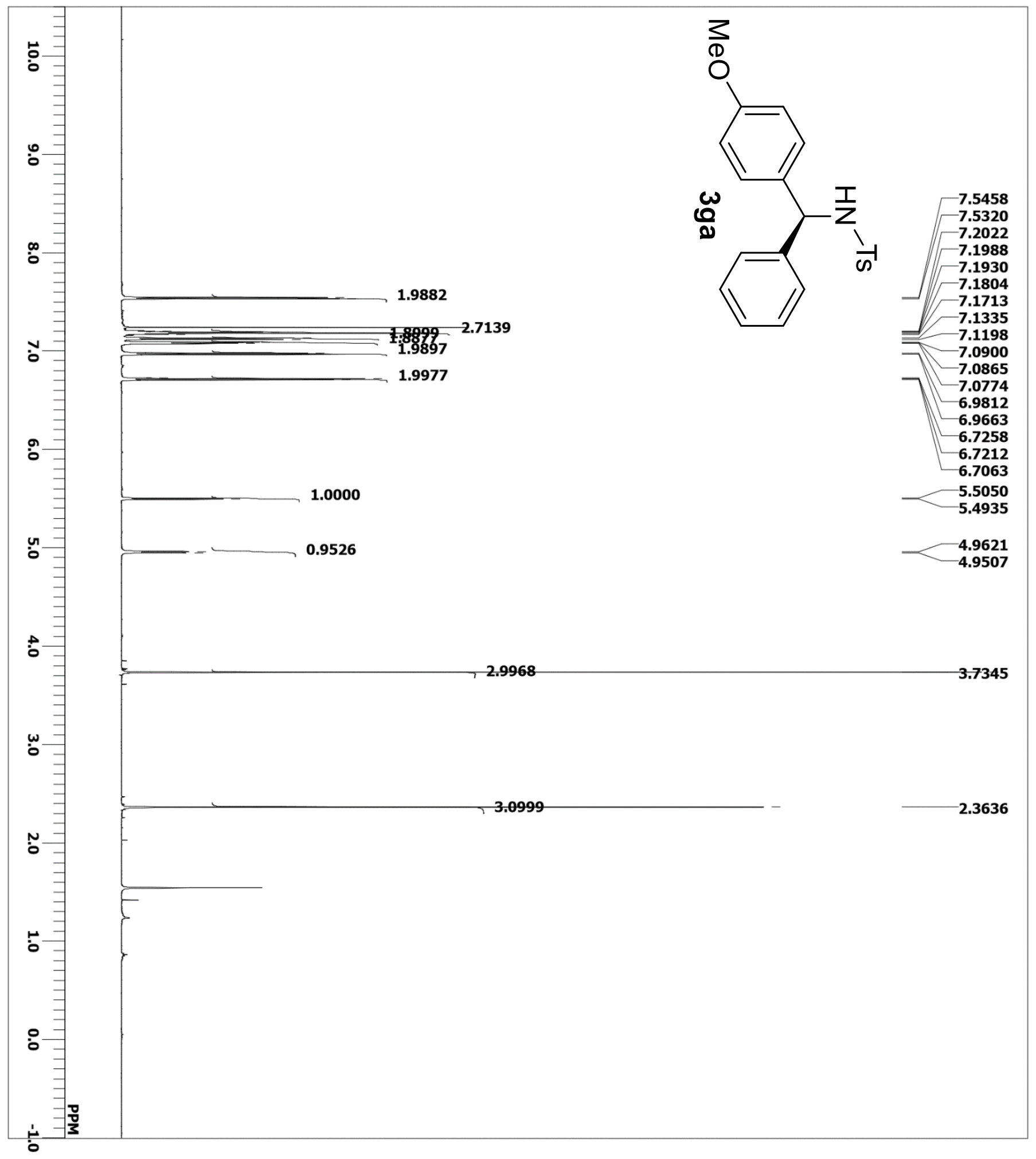




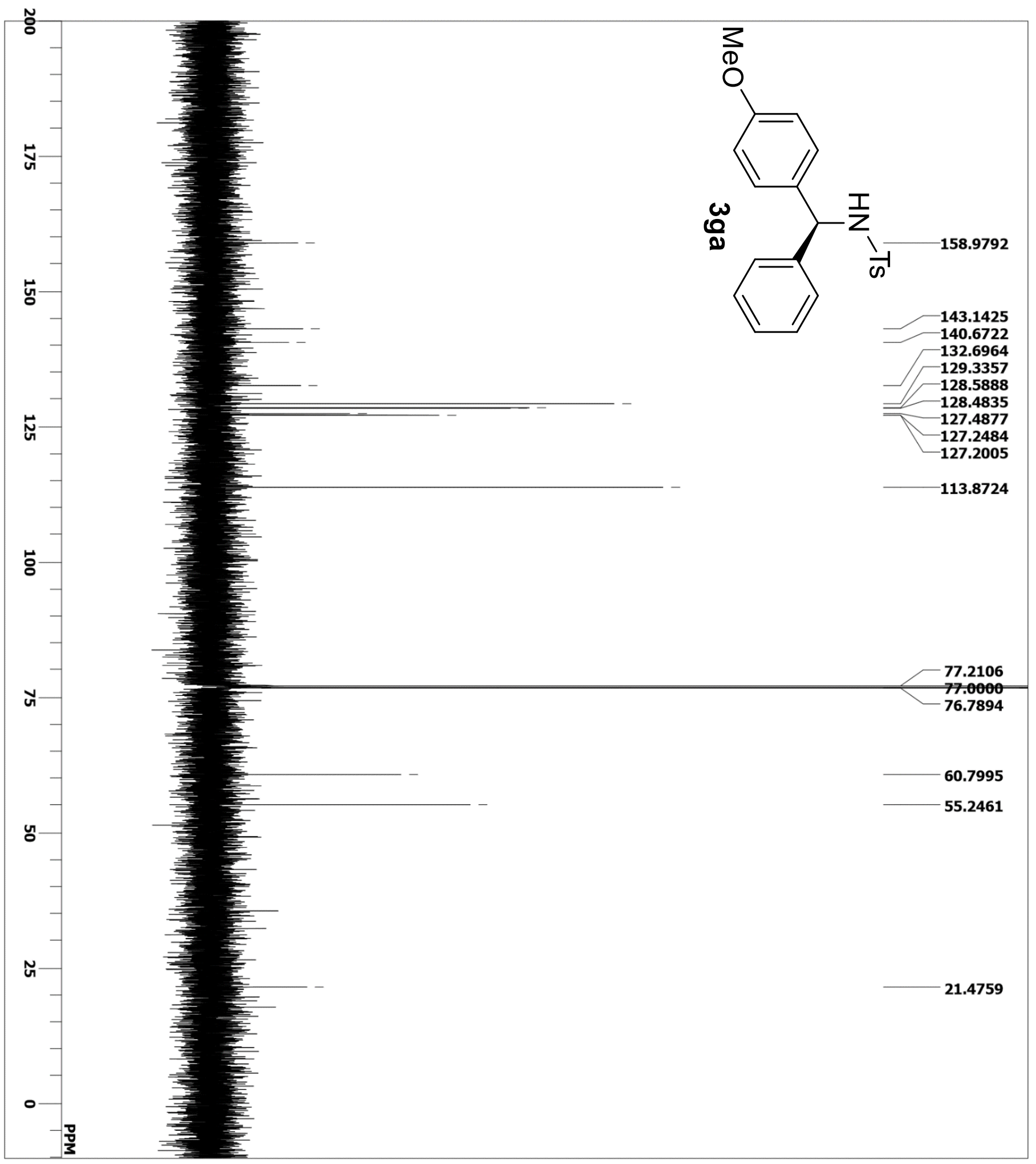


<smiles>Cc1ccc(C(N[TeH])c2cccc(C)c2)cc1</smiles>

$\mathrm{mV}$

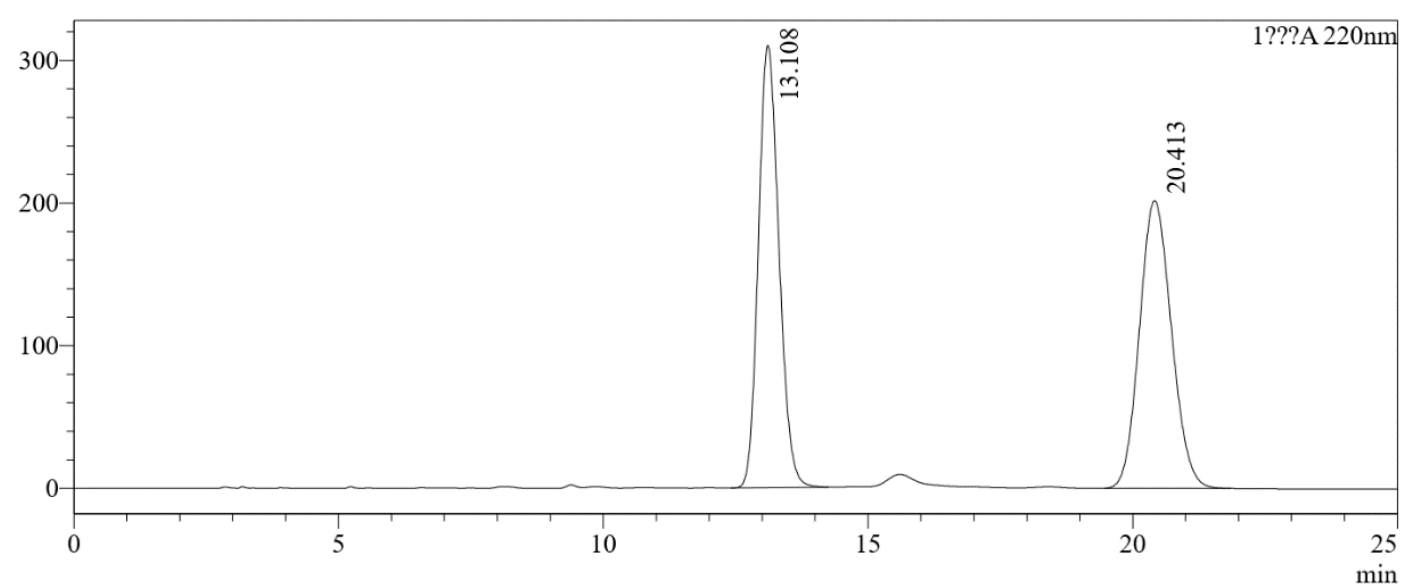

???A 220 nm
\begin{tabular}{|r|r|r|r|r|}
\hline Peak\# & Ret. Time & Area & Height & Area $\%$ \\
\hline 1 & 13.108 & 8388263 & 310232 & 49.699 \\
\hline 2 & 20.413 & 8489891 & 201708 & 50.301 \\
\hline
\end{tabular}<smiles>Cc1ccc(C(N[As])c2cccc(C)c2)cc1</smiles>

$\mathrm{mV}$

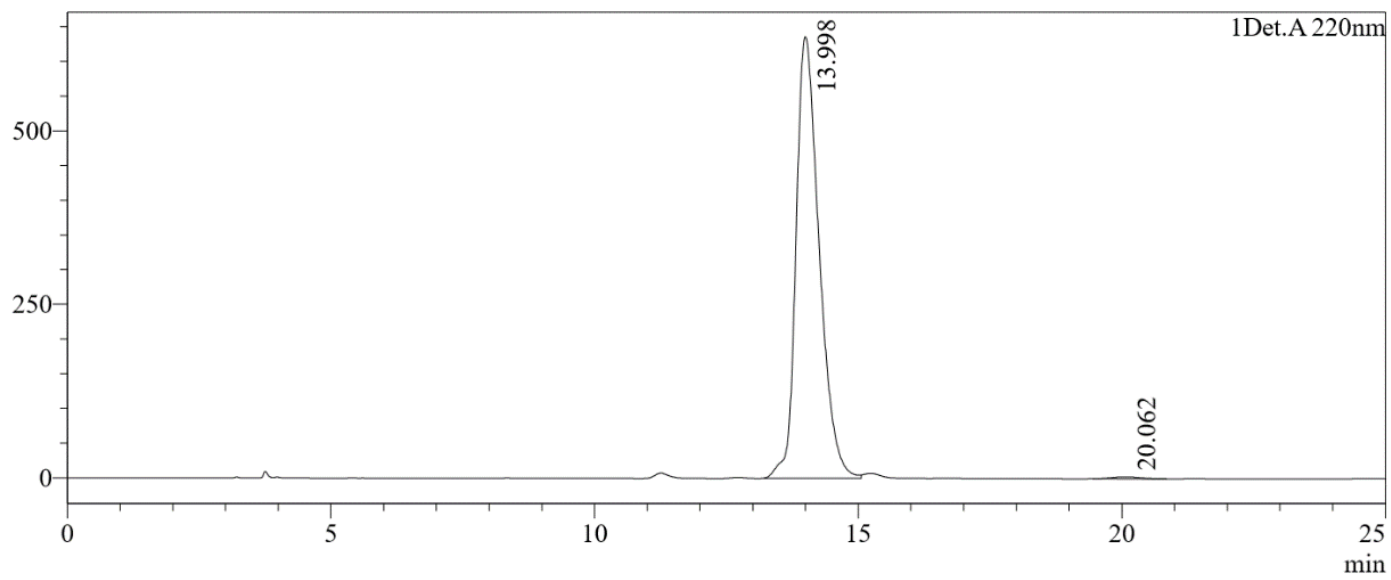

Det.A 220nm

\begin{tabular}{|r|r|r|r|r|}
\hline \multicolumn{1}{|c|}{ Peak\# } & Ret. Time & \multicolumn{1}{c|}{ Area } & \multicolumn{1}{c|}{ Height } & \multicolumn{1}{c|}{ Area\% } \\
\hline 1 & 13.998 & 19411221 & 635701 & 99.464 \\
\hline 2 & 20.062 & 104681 & & \\
\hline
\end{tabular}




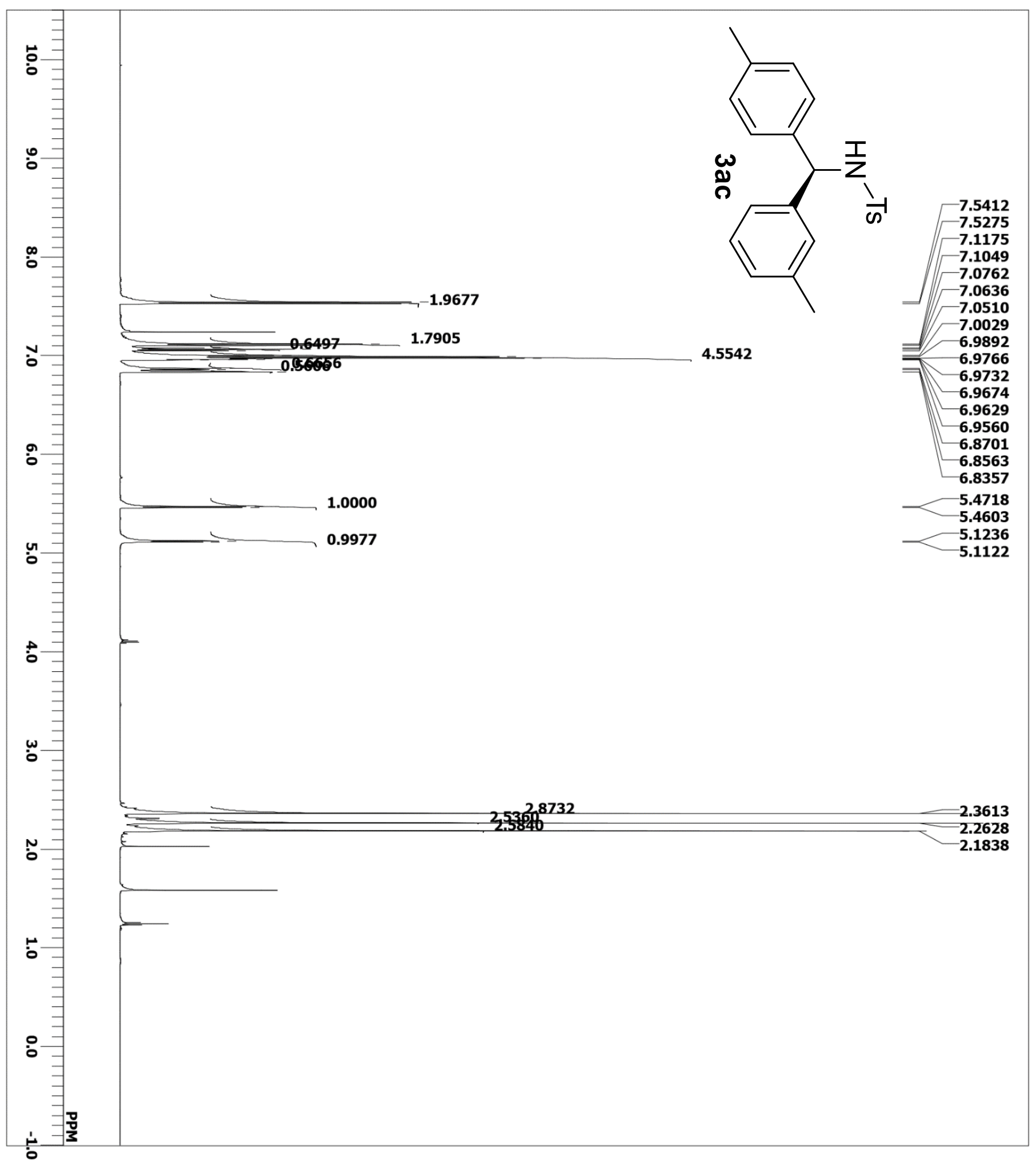




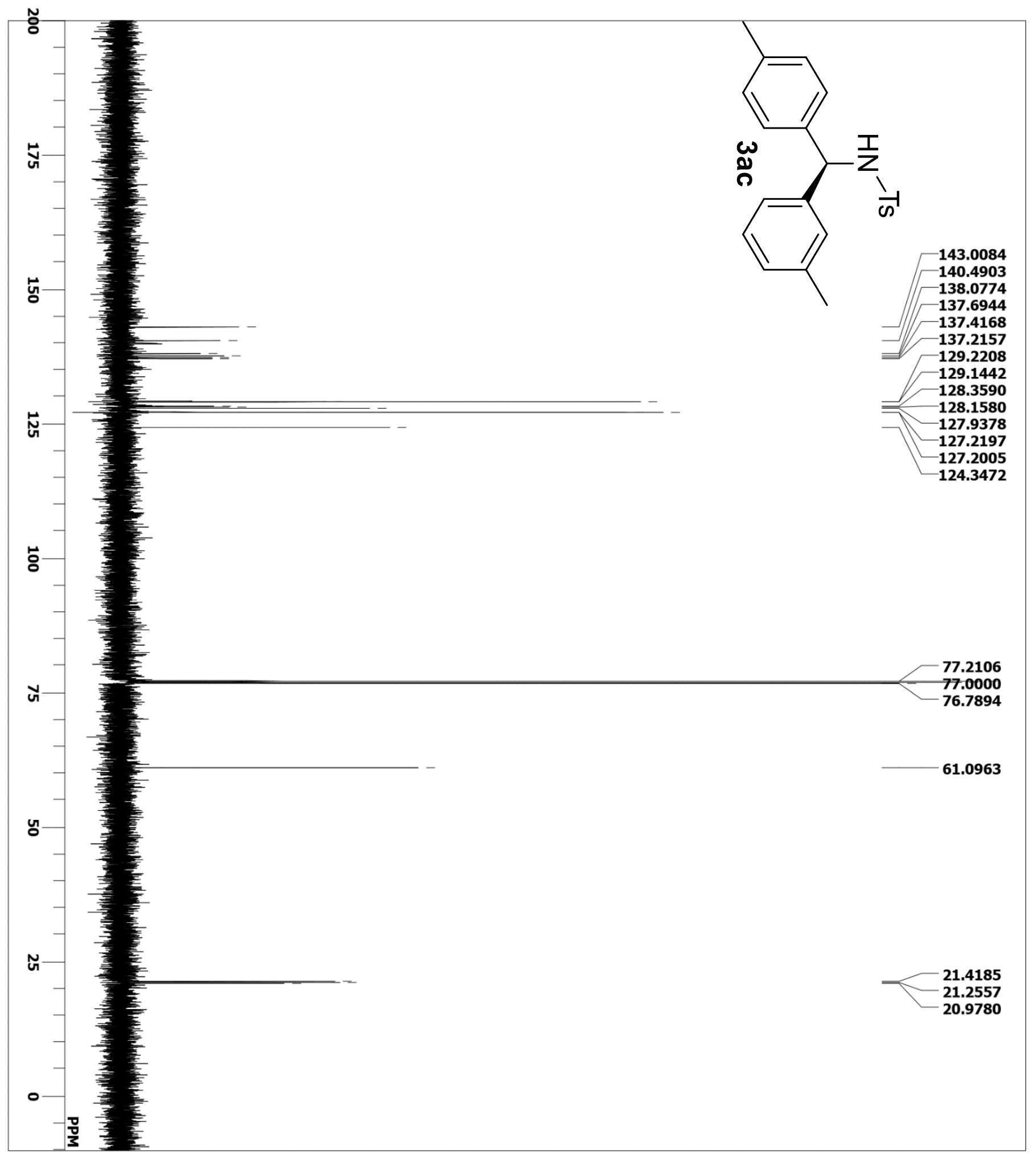


<smiles>Cc1ccc(C(N[As])c2ccccc2C)cc1</smiles>

$\mathrm{mV}$

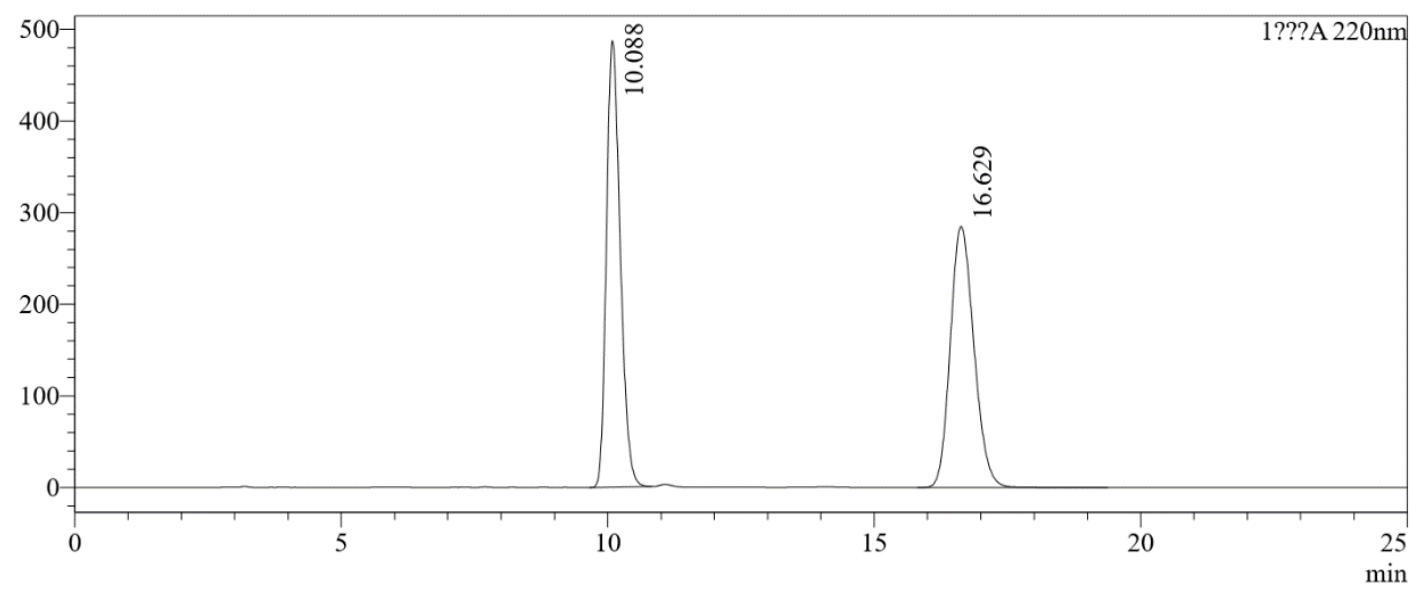

???A 220nm
\begin{tabular}{|r|r|r|r|r|}
\hline Peak\# & Ret. Time & \multicolumn{1}{|c|}{ Area } & \multicolumn{1}{c|}{ Height } & \multicolumn{1}{c|}{ Area $\%$} \\
\hline 1 & 10.088 & 8799958 & 487298 & 49.970 \\
\hline 2 & 16.629 & 8810481 & 285293 & 50.030 \\
\hline
\end{tabular}<smiles>[3H]NC(c1ccc(C)cc1)c1ccccc1C</smiles>

$\mathrm{mV}$

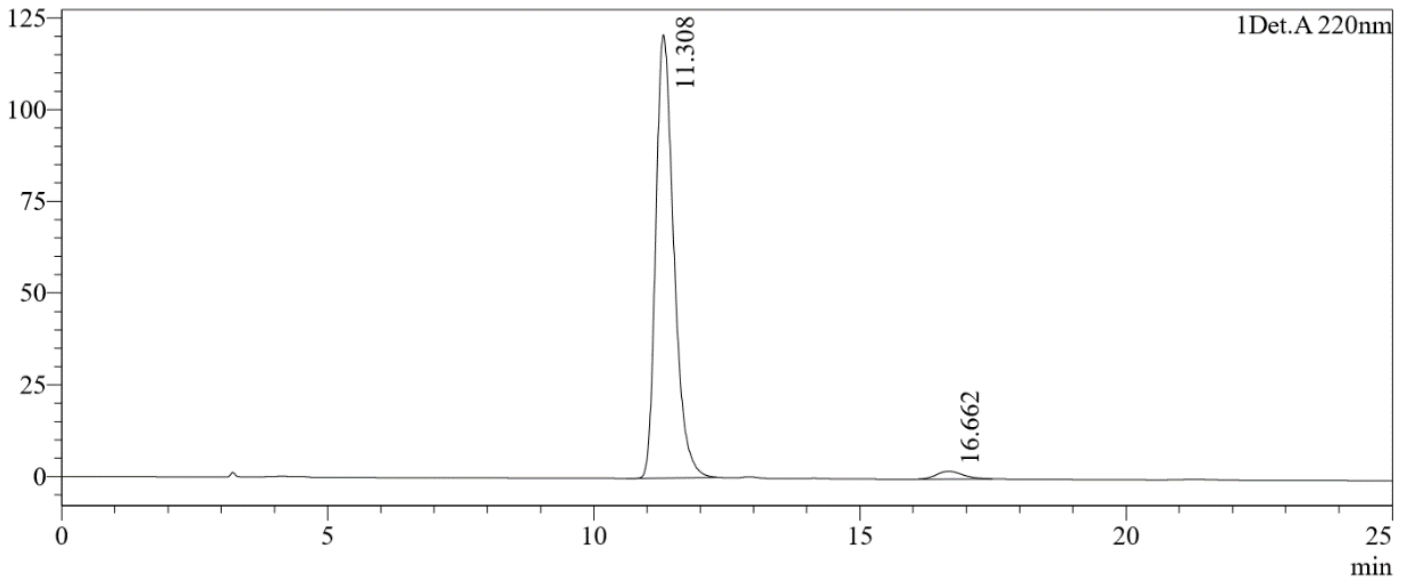

Det.A 220nm
\begin{tabular}{|r|r|r|r|r|}
\hline Peak\# & Ret. Time & \multicolumn{1}{c|}{ Area } & \multicolumn{1}{c|}{ Height } & \multicolumn{1}{c|}{ Area $\%$} \\
\hline 1 & 11.308 & 2848417 & 120930 & 97.488 \\
\hline 2 & 16.662 & 73399 & 2115 & 2.512 \\
\hline
\end{tabular}




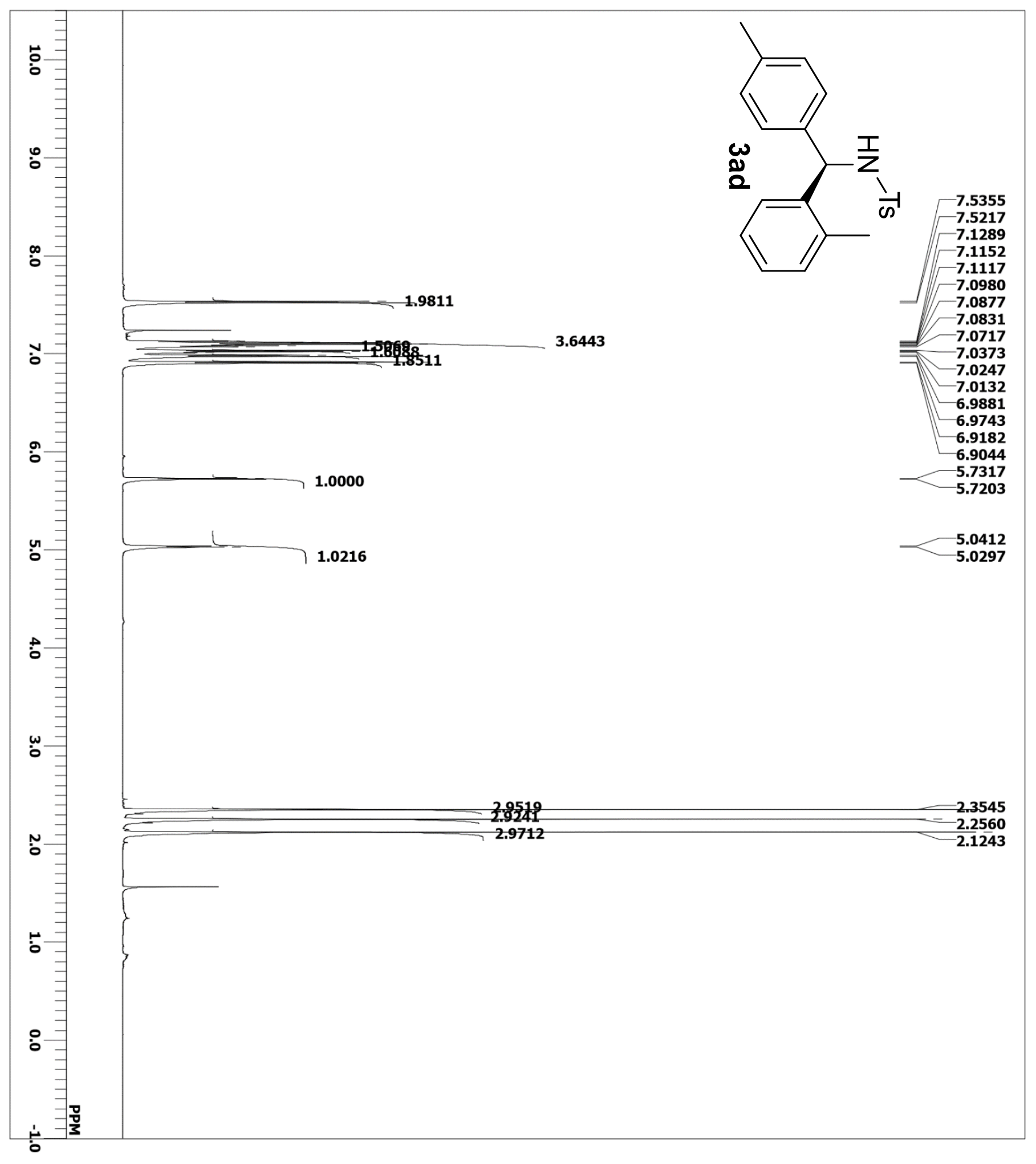




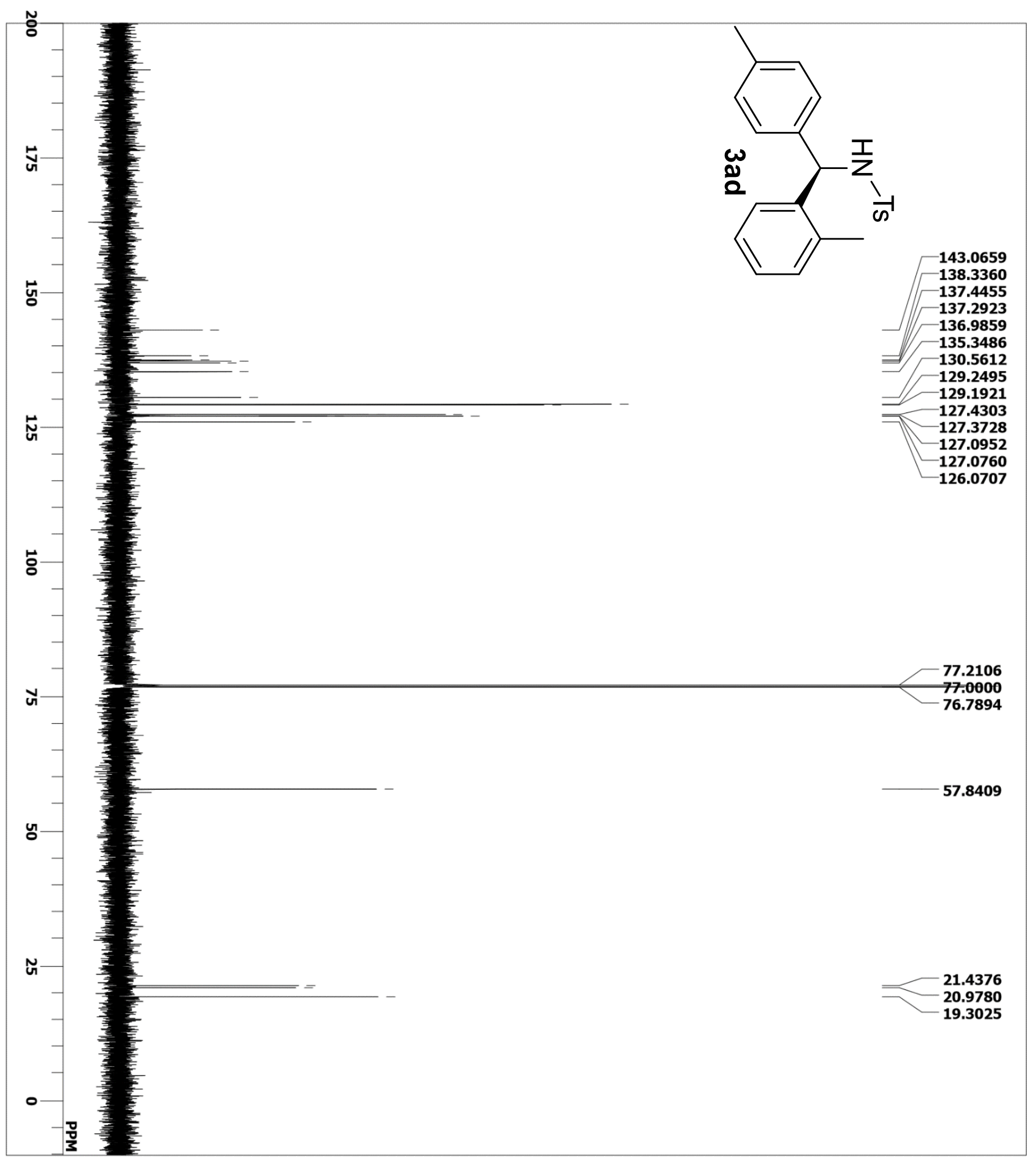


<smiles>Cc1ccc(C(NC(F)(F)F)c2ccc(F)cc2)cc1</smiles>

$\mathrm{mV}$

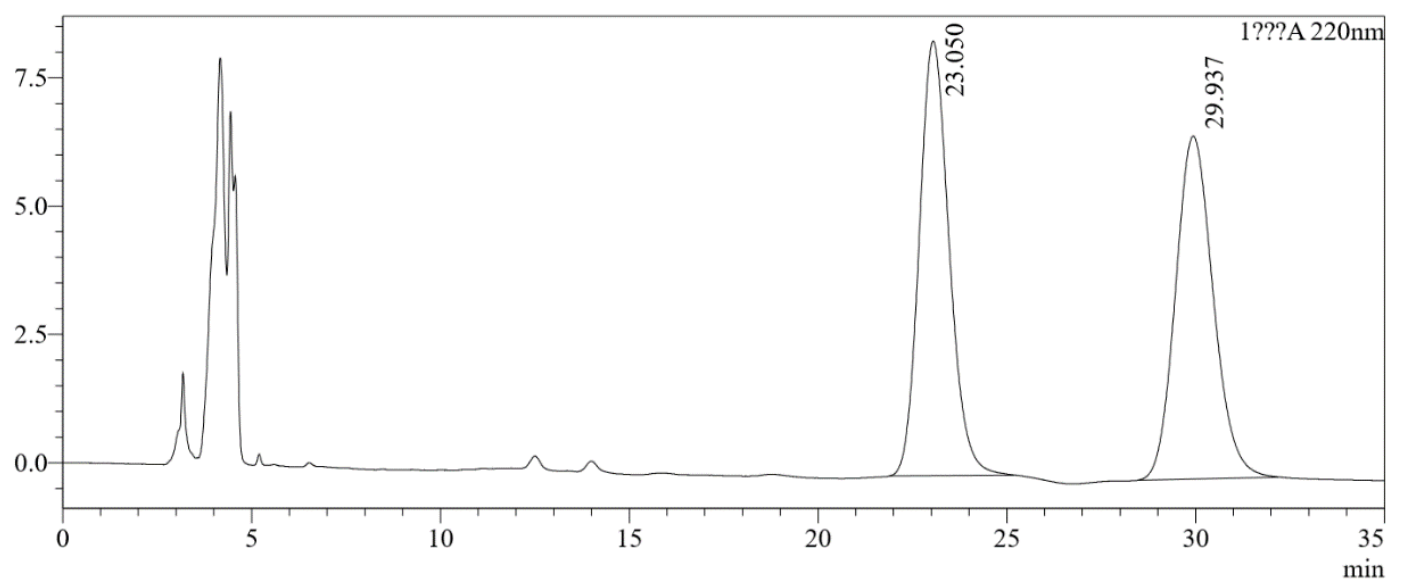

???A $220 \mathrm{~nm}$
\begin{tabular}{|r|r|r|r|r|}
\hline Peak\# & Ret. Time & Area & Height & \multicolumn{1}{c|}{ Area $\%$} \\
\hline 1 & 23.050 & 465668 & 8467 & 50.109 \\
\hline 2 & 29.937 & 463651 & 6679 & 49.891 \\
\hline
\end{tabular}

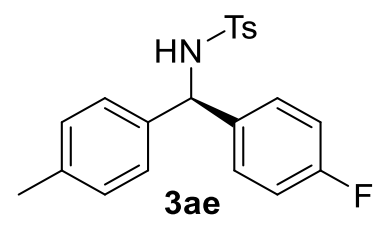

$\mathrm{mV}$

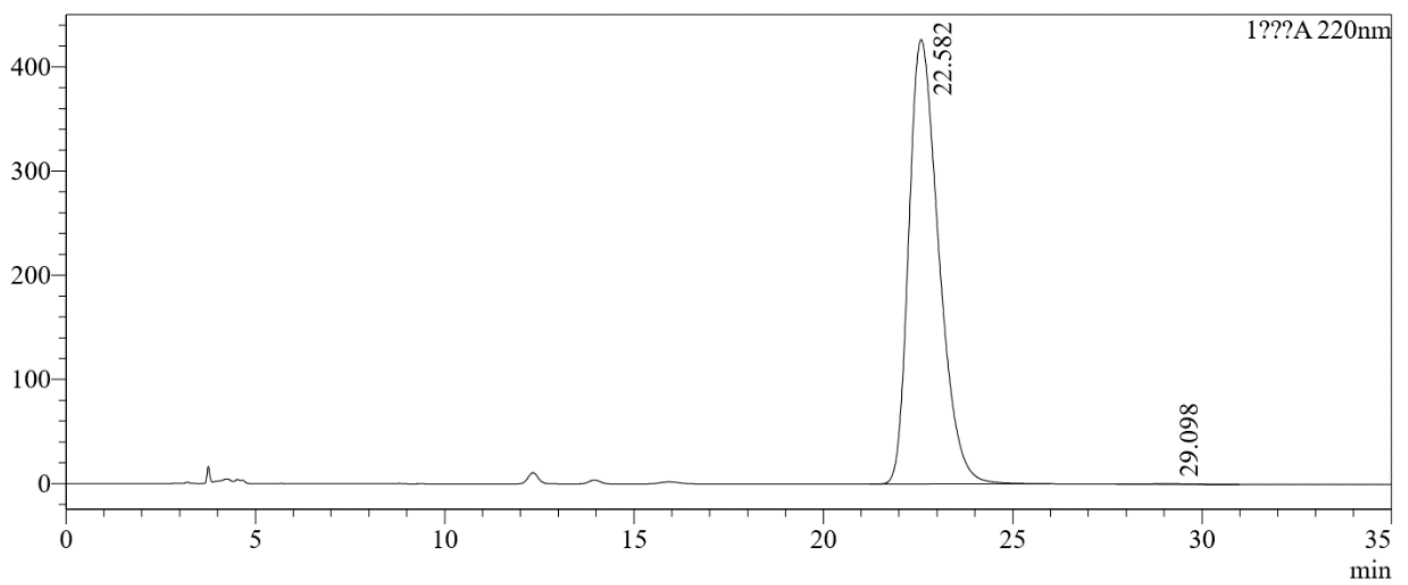

???A 220nm
\begin{tabular}{|r|r|r|r|r|}
\hline Peak\# & Ret. Time & \multicolumn{1}{c|}{ Area } & Height & \multicolumn{1}{c|}{ Area $\%$} \\
\hline 1 & 22.582 & 23498301 & 426748 & 99.872 \\
\hline 2 & 29.098 & 30118 & 481 & 0.128 \\
\hline
\end{tabular}




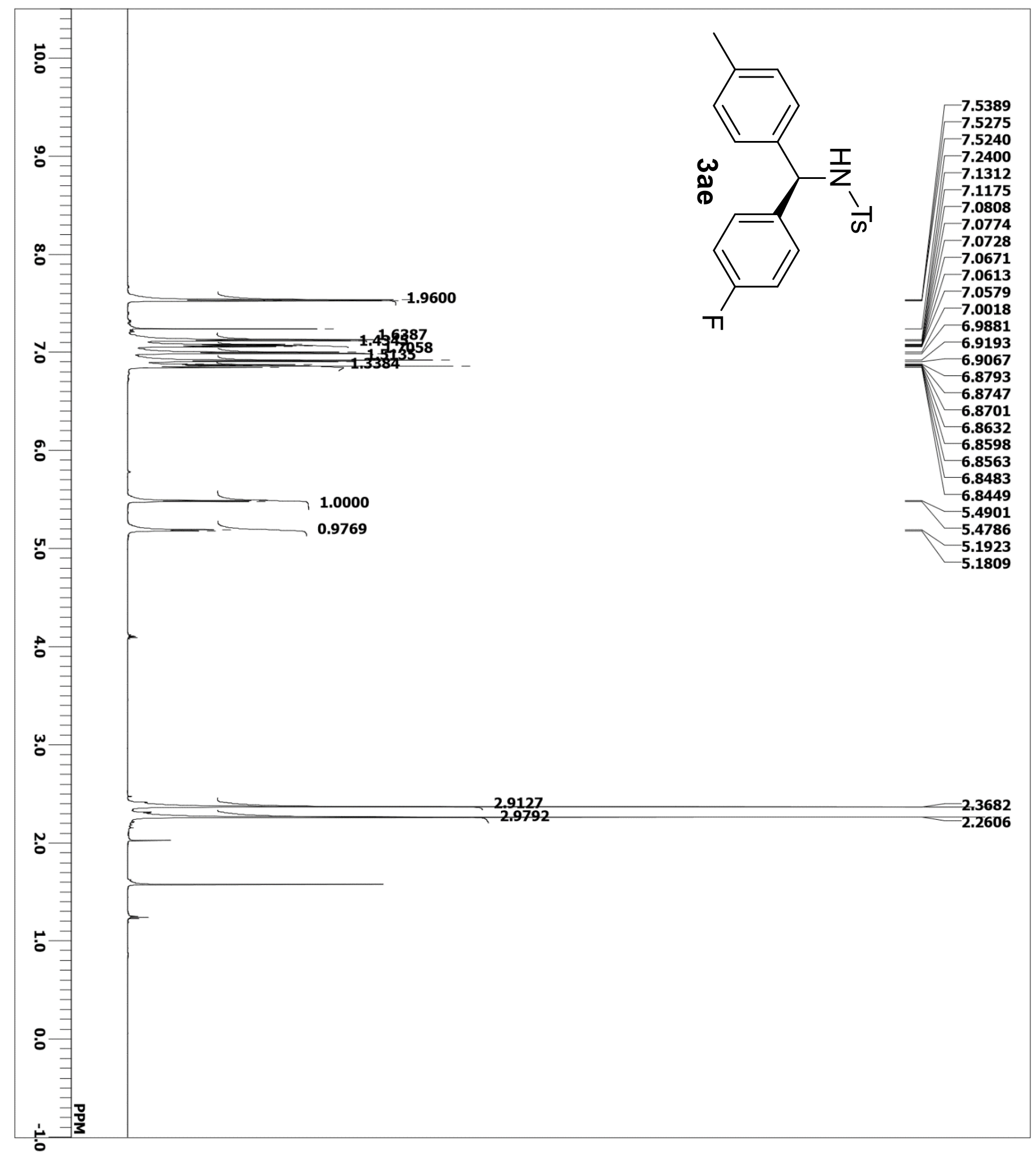




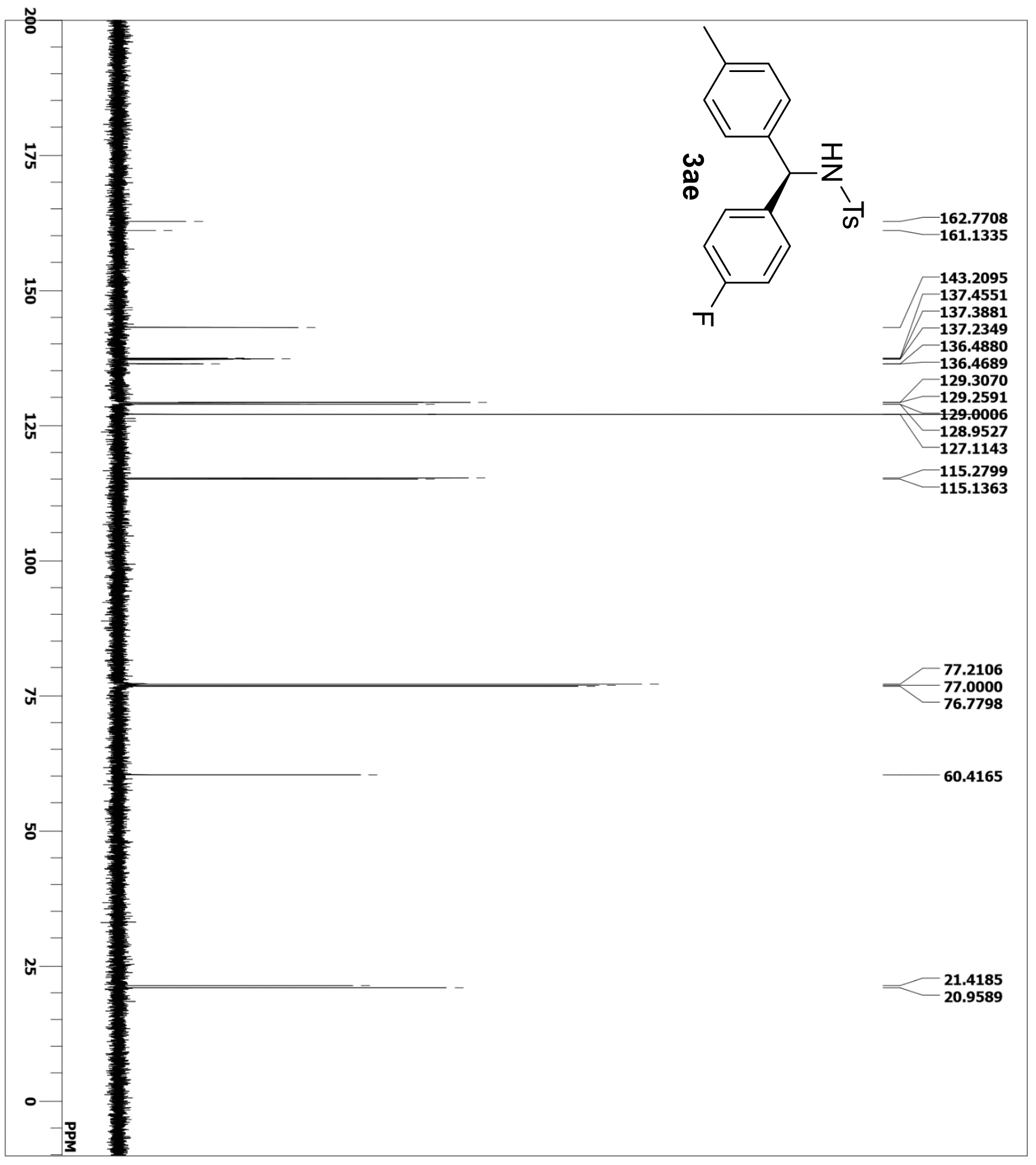


<smiles>Cc1ccc(C(NS)c2ccc(Cl)cc2)cc1</smiles>

$\mathrm{mV}$
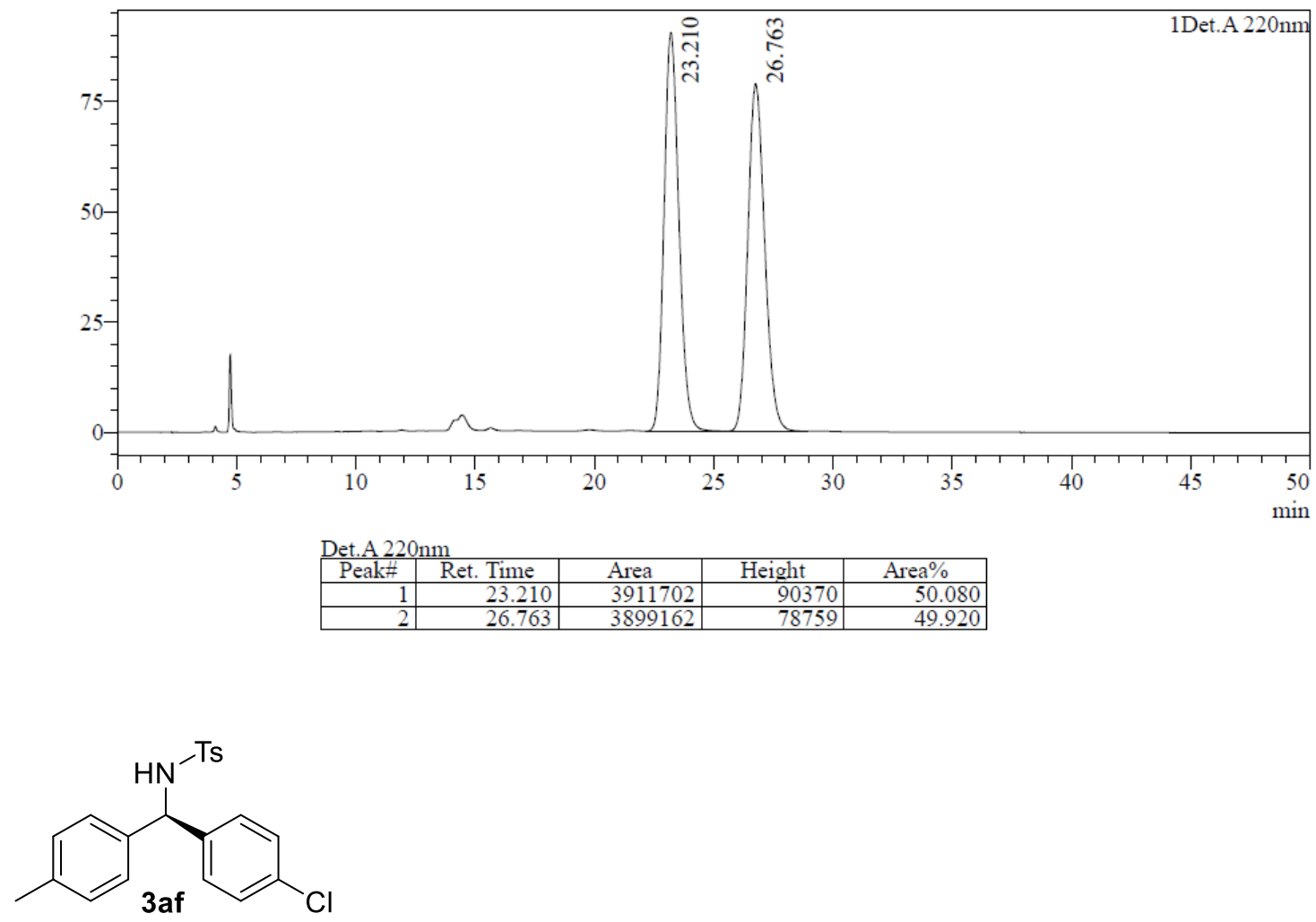

$\mathrm{mV}$

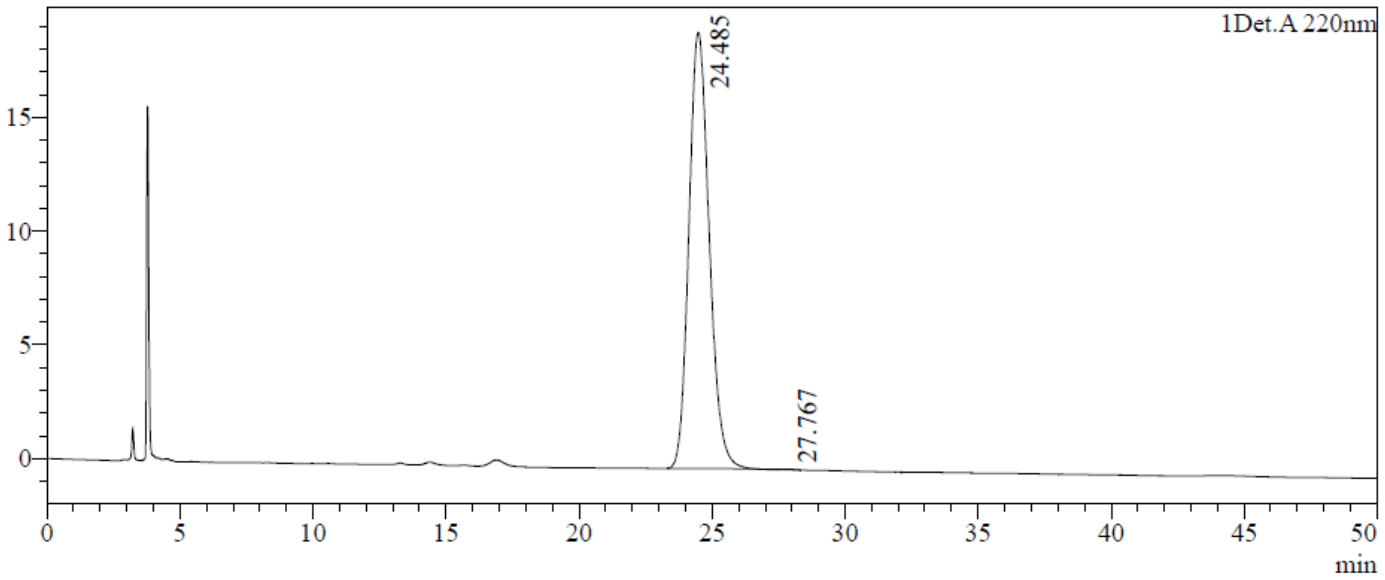

Det.A 220 nm
\begin{tabular}{|r|r|r|r|r|}
\hline Peak\# & Ret. Time & \multicolumn{1}{c|}{ Area } & Height & \multicolumn{1}{c|}{ Area $\%$} \\
\hline 1 & 24.485 & 1018713 & 19181 & 99.917 \\
\hline 2 & 27.767 & 849 & 23 & 0.083 \\
\hline
\end{tabular}




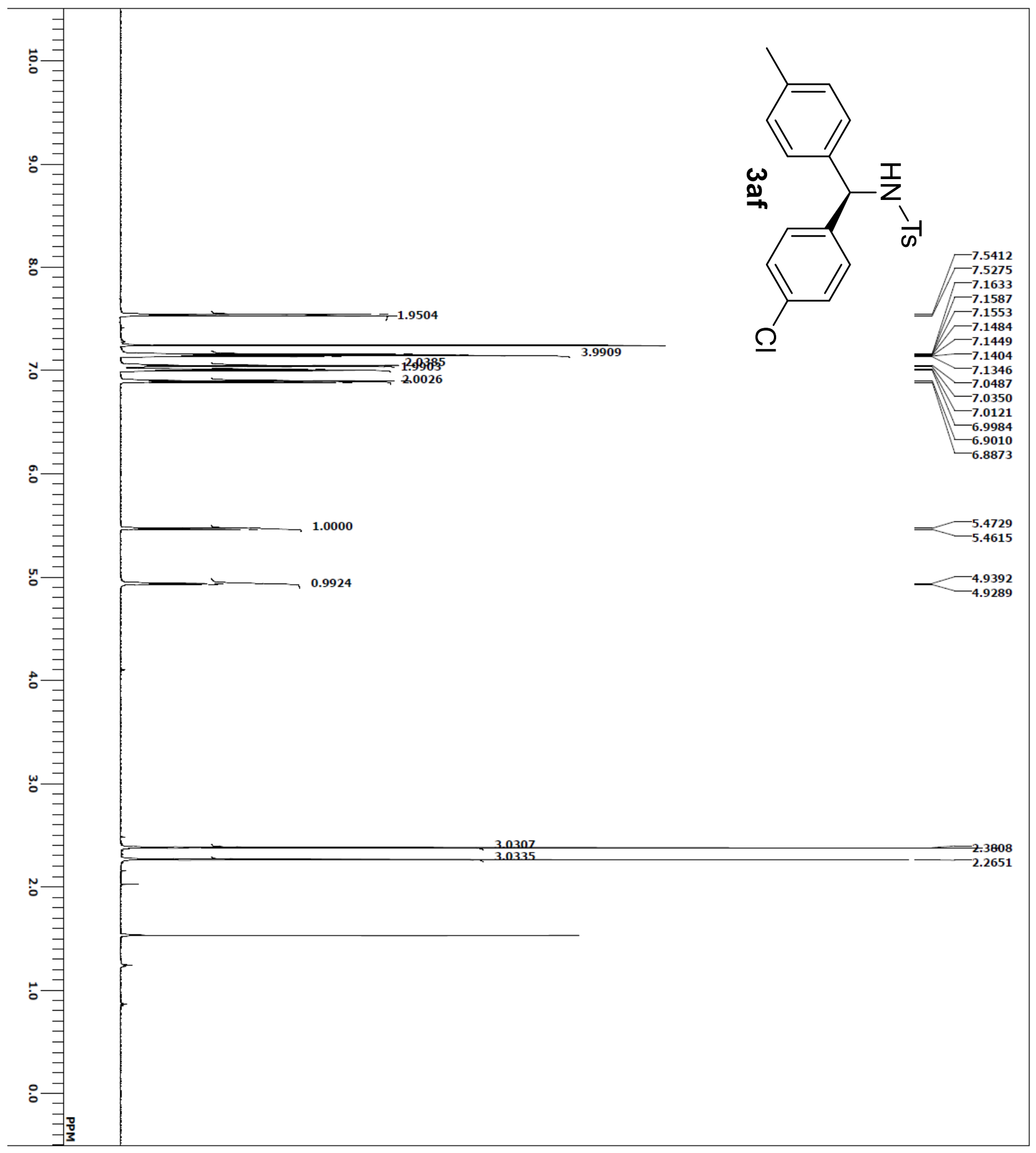




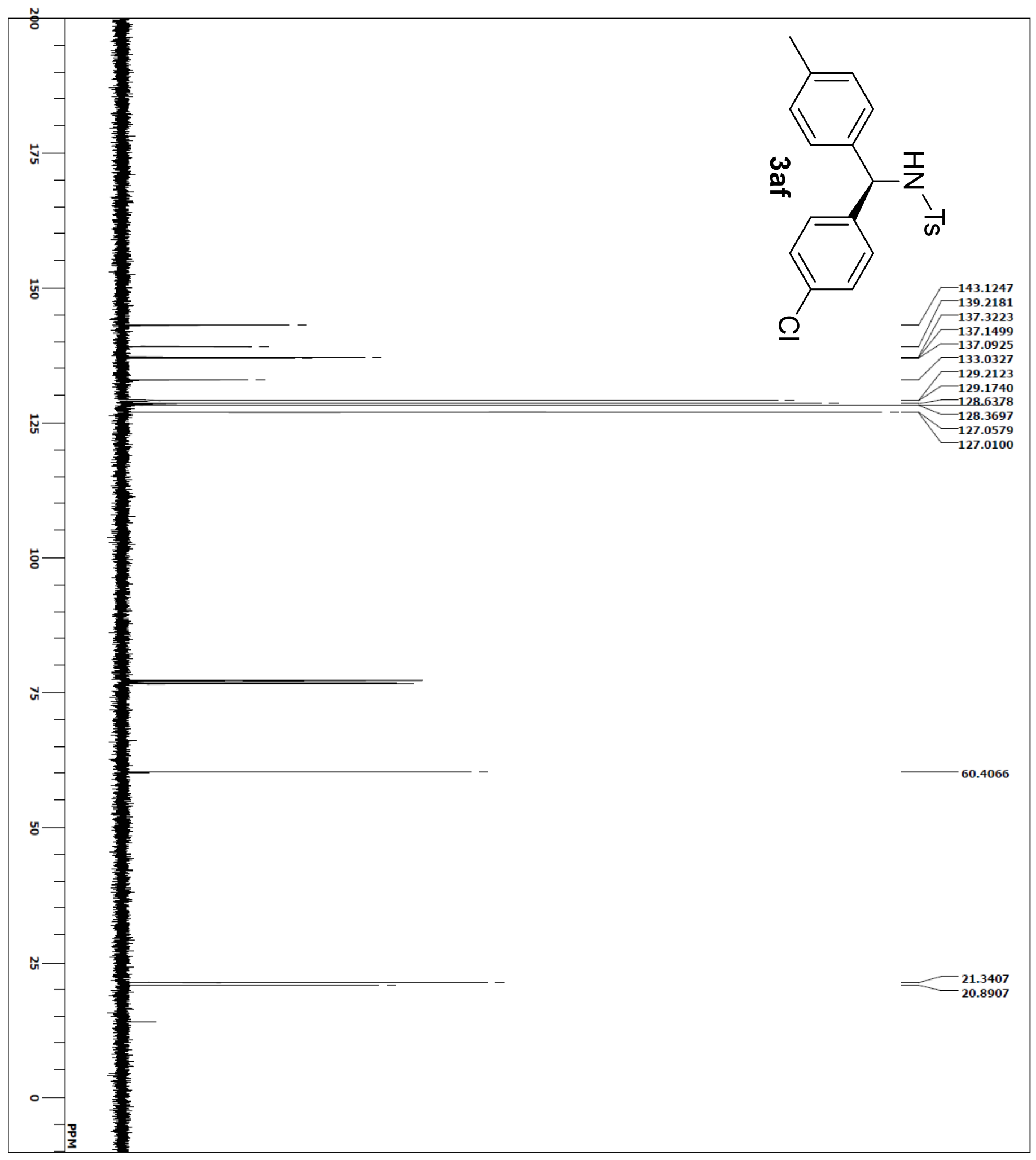


<smiles>Cc1ccc(C(NS)c2ccc(Br)cc2)cc1</smiles>

$\mathrm{mV}$

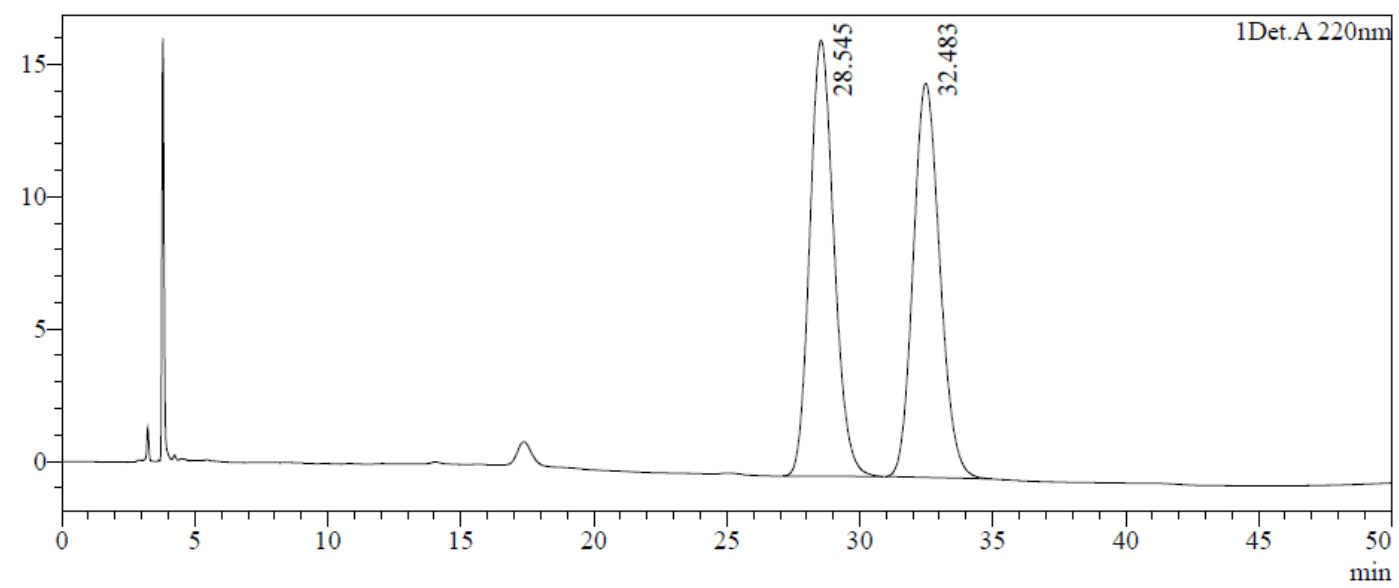

Det.A $220 \mathrm{~nm}$
\begin{tabular}{|r|r|r|r|r|}
\hline \multicolumn{1}{|c|}{ Peak\# } & Ret. Time & Area & Height & \multicolumn{1}{c|}{ Area $\%$} \\
\hline 1 & 28.545 & 1041023 & 16451 & 50.111 \\
\hline 2 & 32.483 & 1036417 & 14878 & 49.889 \\
\hline
\end{tabular}

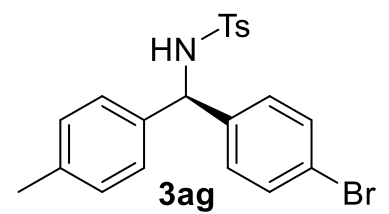

$\mathrm{mV}$

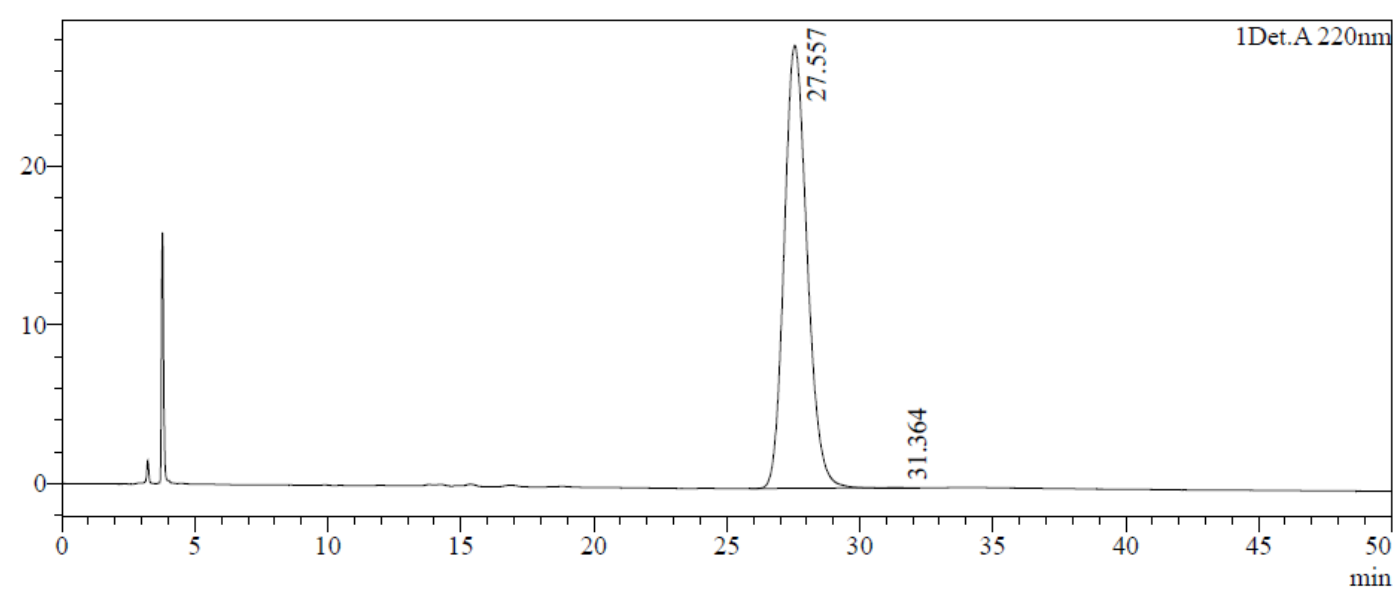

Det.A 220nm
\begin{tabular}{|r|r|r|r|r|}
\hline Peak\# & Ret. Time & Area & Height & \multicolumn{1}{c|}{ Area $\%$} \\
\hline 1 & 27.557 & 1672235 & 27931 & 99.906 \\
\hline 2 & 31.364 & 1577 & 32 & 0.094 \\
\hline
\end{tabular} 


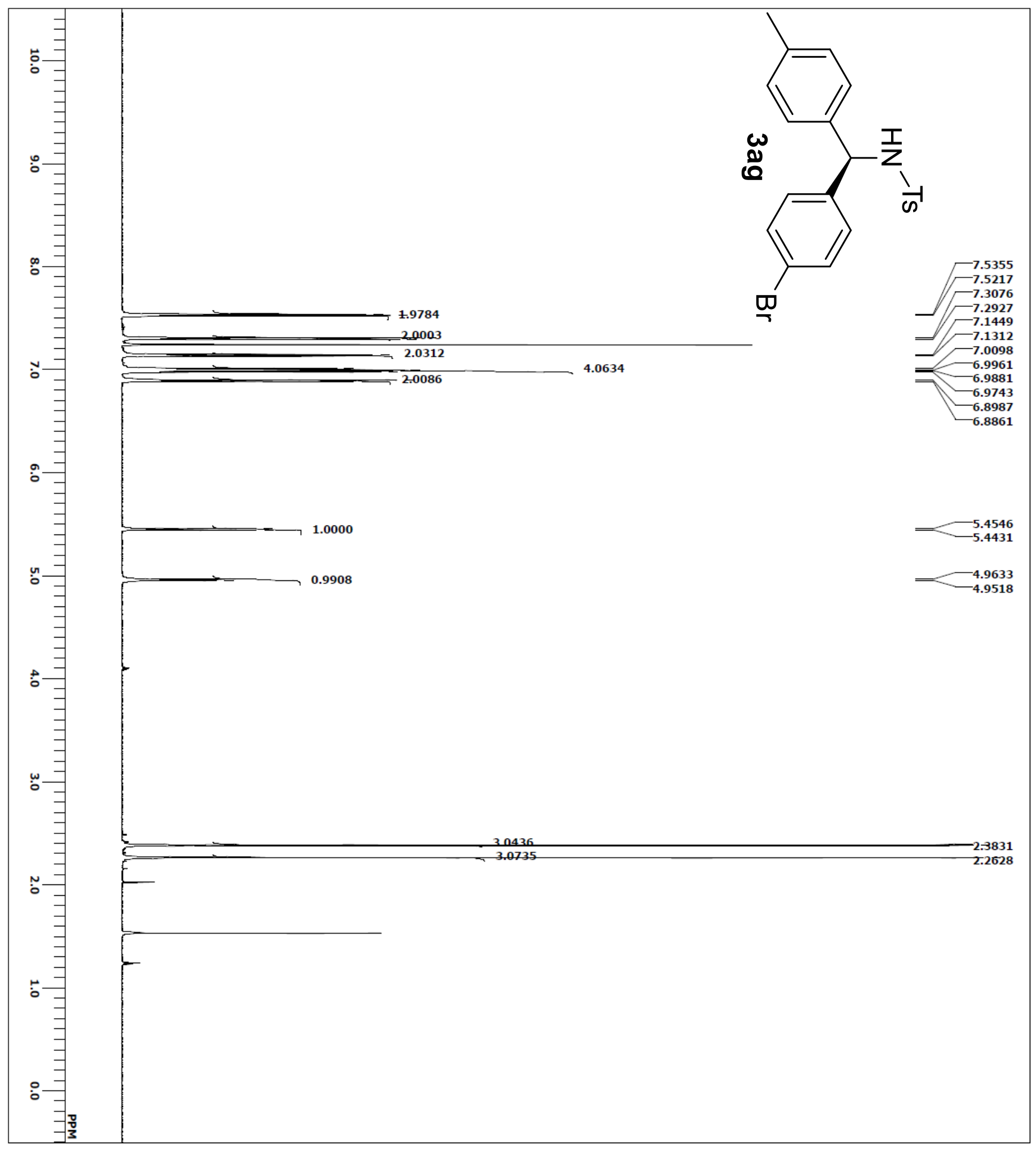




$$
1
$$


<smiles>COc1ccc(C(N[TeH])c2ccc(C)cc2)cc1</smiles>

$\mathrm{mV}$

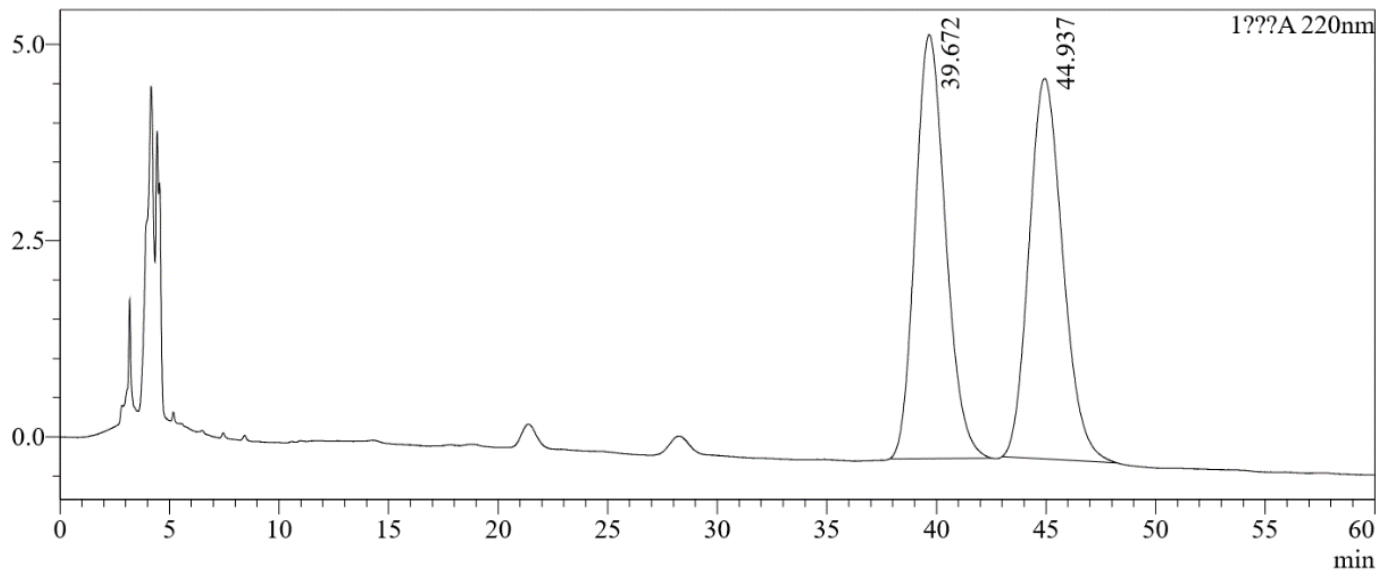

???A 220nm
\begin{tabular}{|r|r|r|r|r|}
\hline Peakt & Ret. Time & Area & Height & \multicolumn{1}{|c|}{ Area $\%$} \\
\hline 1 & 39.672 & 520091 & 5400 & 49.996 \\
\hline 2 & 44.937 & 520165 & 4847 & 50.004 \\
\hline
\end{tabular}<smiles>COc1ccc(C(N[TeH])c2ccc(C)cc2)cc1</smiles>

$\mathrm{mV}$

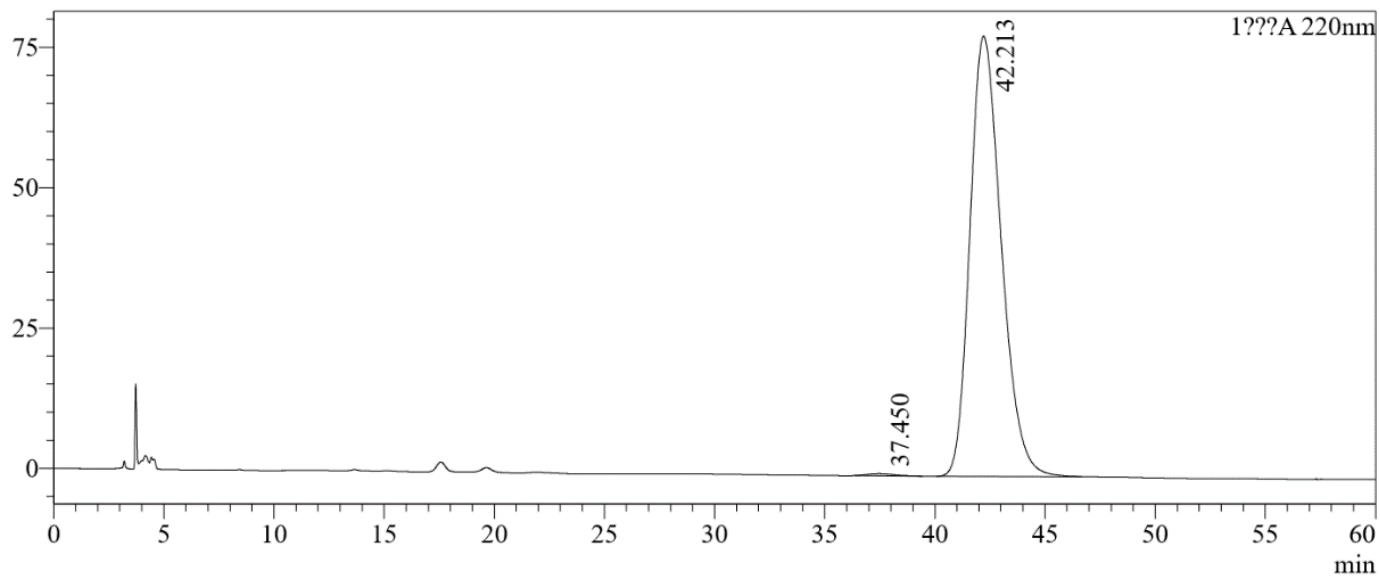

???A $220 \mathrm{~nm}$
\begin{tabular}{|r|r|r|r|r|}
\hline Peak\# & Ret. Time & Area & Height & \multicolumn{1}{|c|}{ Area $\%$} \\
\hline 1 & 37.450 & 31648 & 377 & 0.406 \\
\hline 2 & 42.213 & 7756410 & 78429 & 99.594 \\
\hline
\end{tabular}




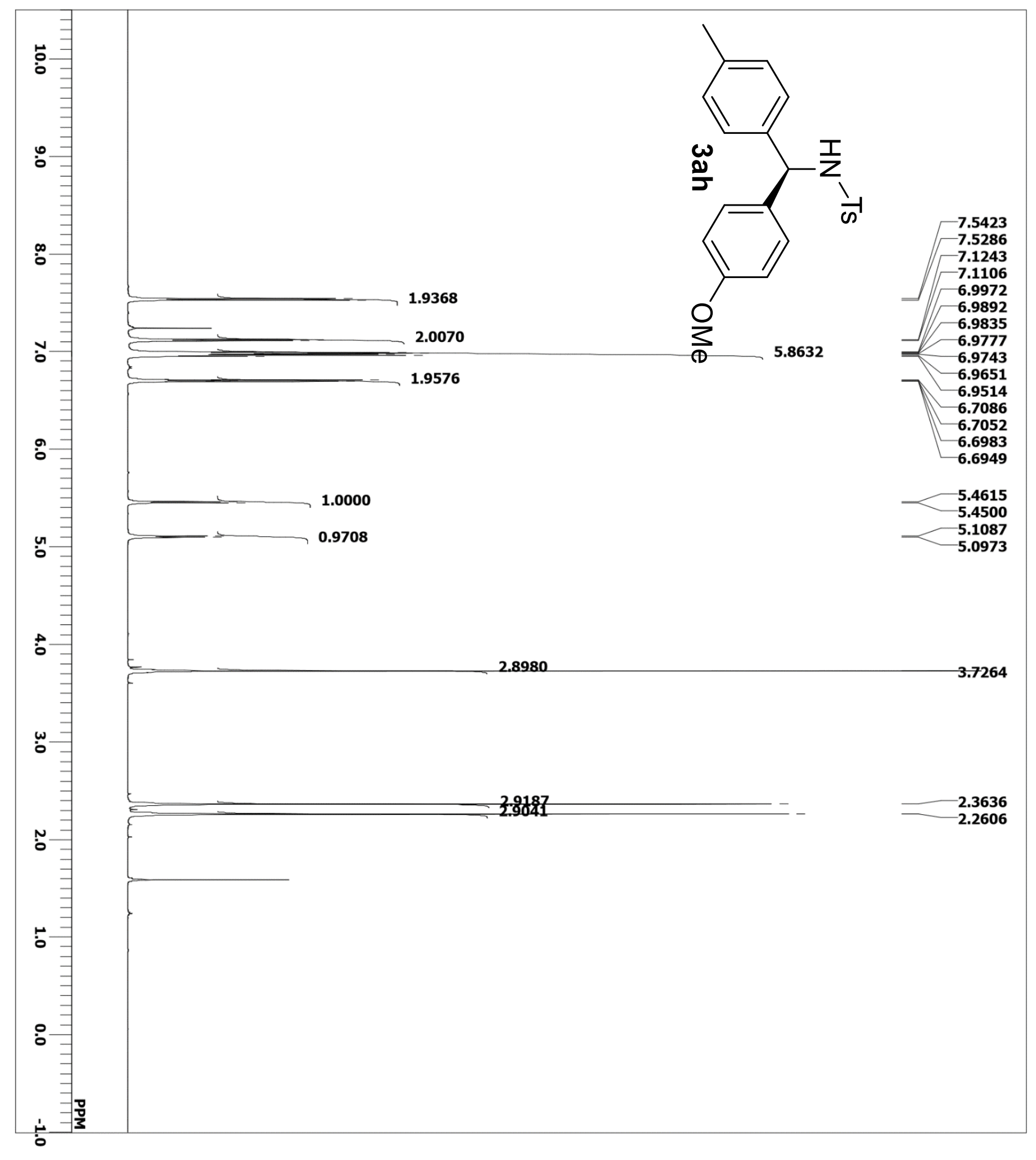




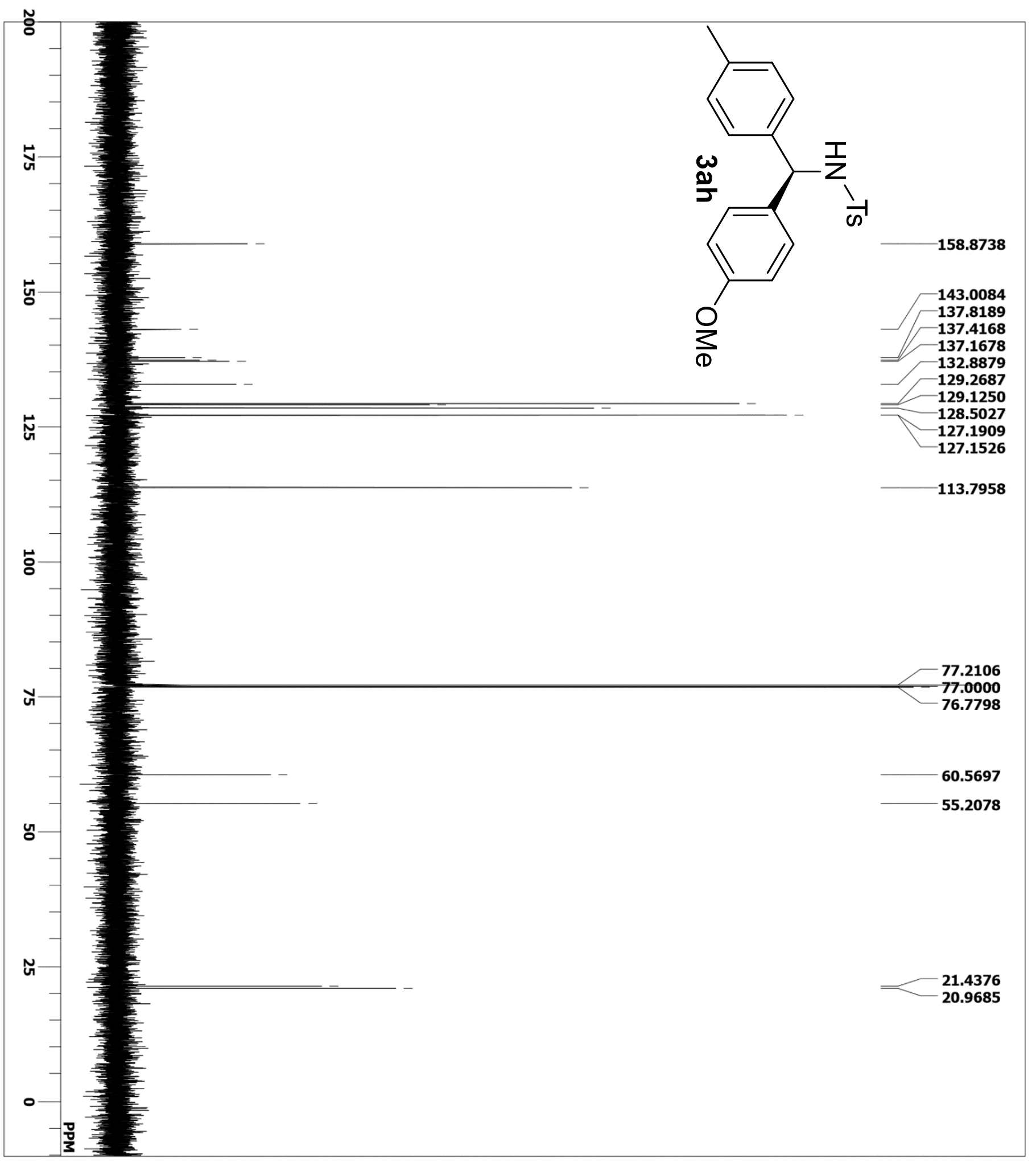


$\underbrace{(N N}_{3 i a}$

$\mathrm{mV}$

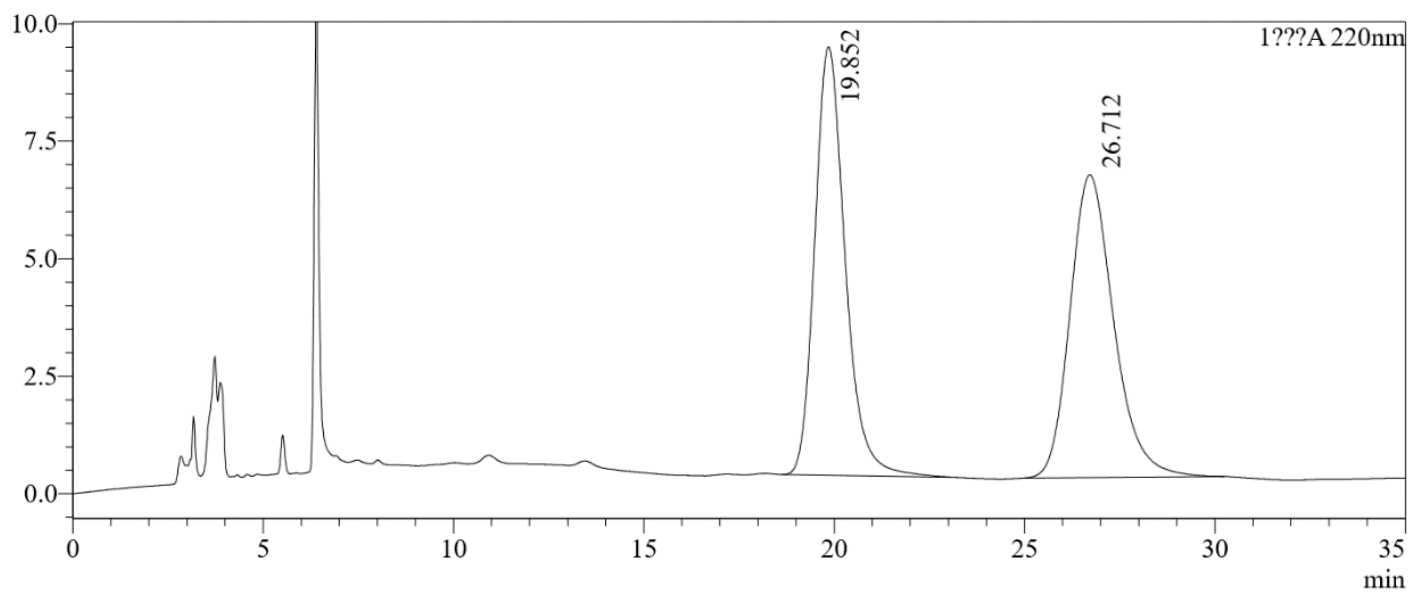

???A 220nm
\begin{tabular}{|r|r|r|r|r|}
\hline Peak\# & Ret. Time & Area & Height & \multicolumn{1}{|c|}{ Area $\%$} \\
\hline 1 & 19.852 & 506021 & 9113 & 49.561 \\
\hline 2 & 26.712 & 514993 & 6438 & 50.439 \\
\hline
\end{tabular}<smiles>[As]N[C@@H](c1ccccc1)c1cccc2ccccc12</smiles>

$\mathrm{mV}$

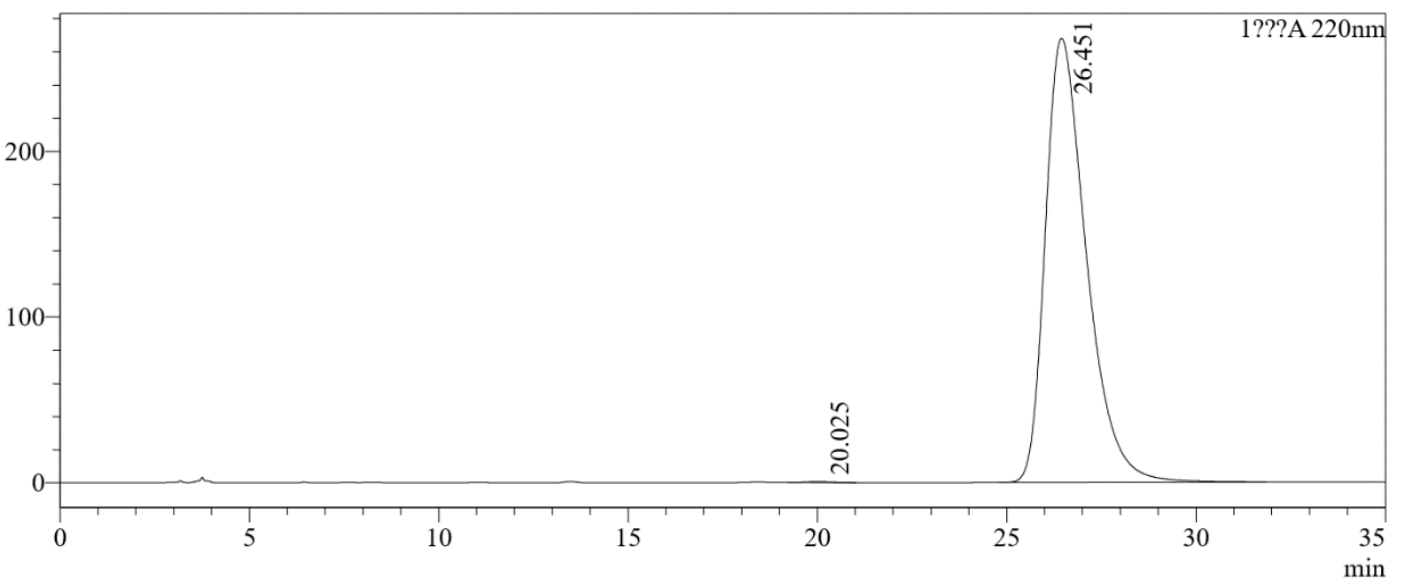

???A 220nm
\begin{tabular}{|r|r|r|r|r|}
\hline Peak\# & Ret. Time & \multicolumn{1}{c|}{ Area } & Height & \multicolumn{1}{c|}{ Area $\%$} \\
\hline 1 & 20.025 & 29152 & 590 & 0.144 \\
\hline 2 & 26.451 & 20254511 & 267898 & 99.856 \\
\hline
\end{tabular}




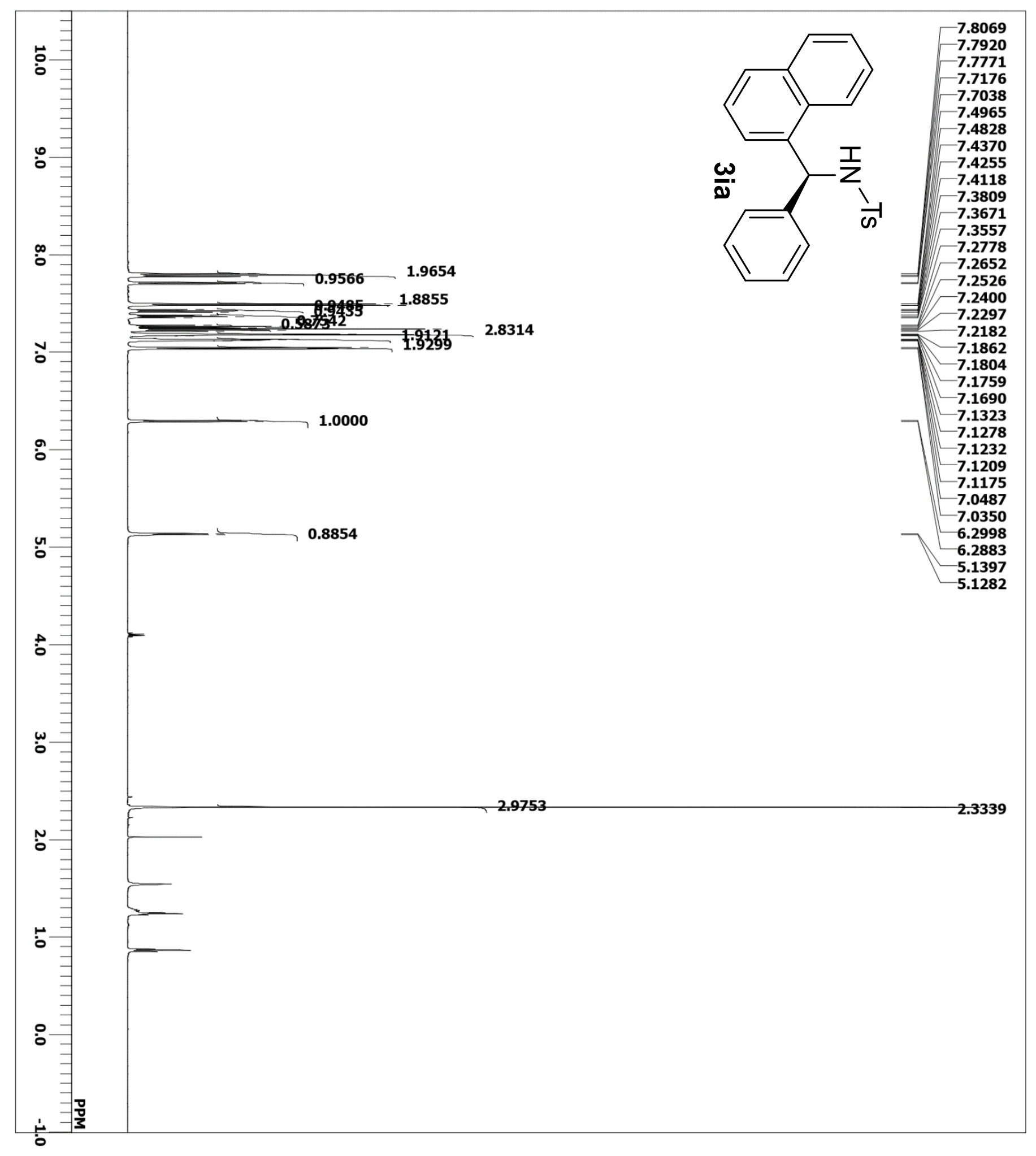




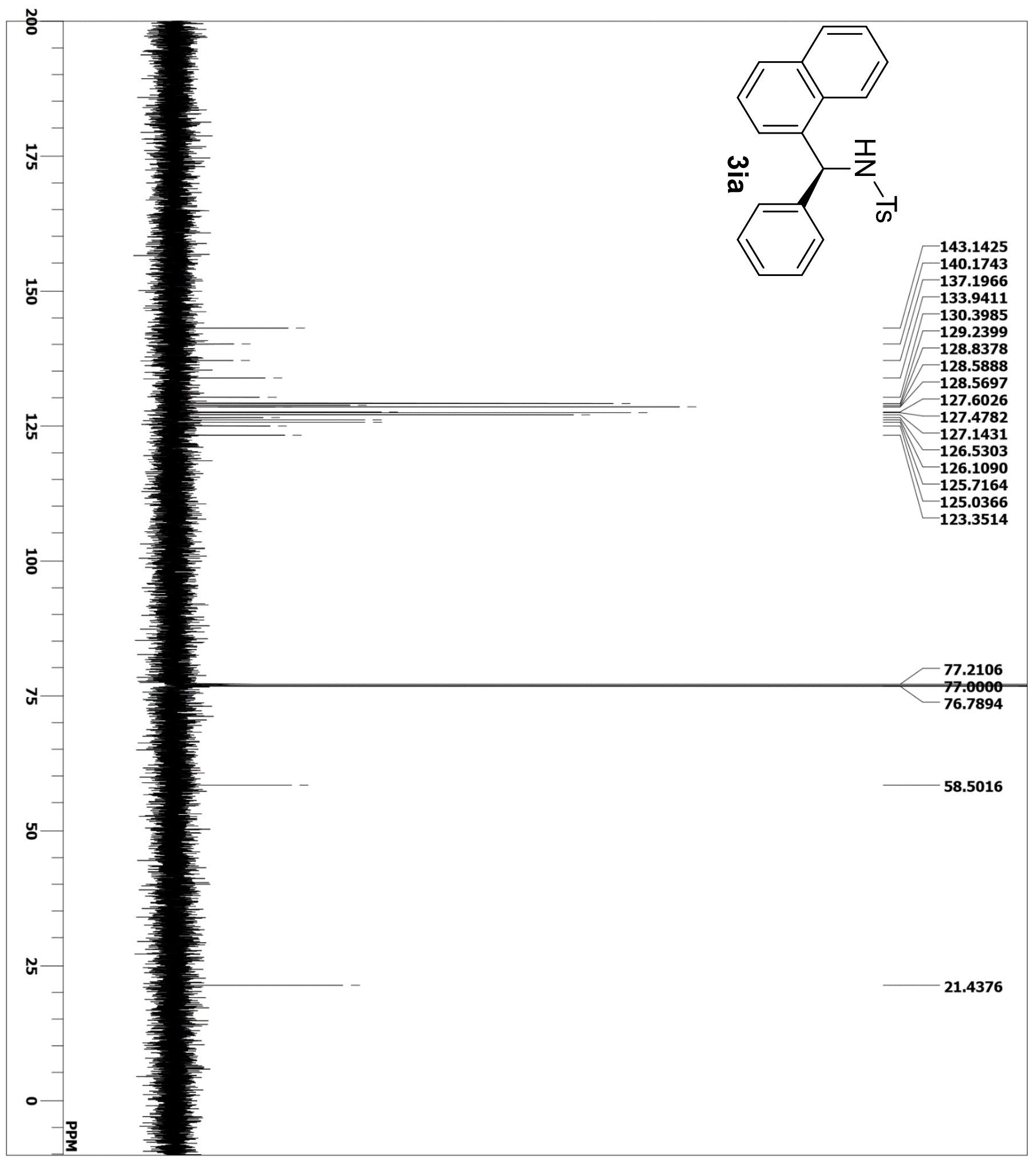


<smiles>[134I]NC(c1ccccc1)c1ccc2ccccc2c1</smiles>

$\mathrm{mV}$

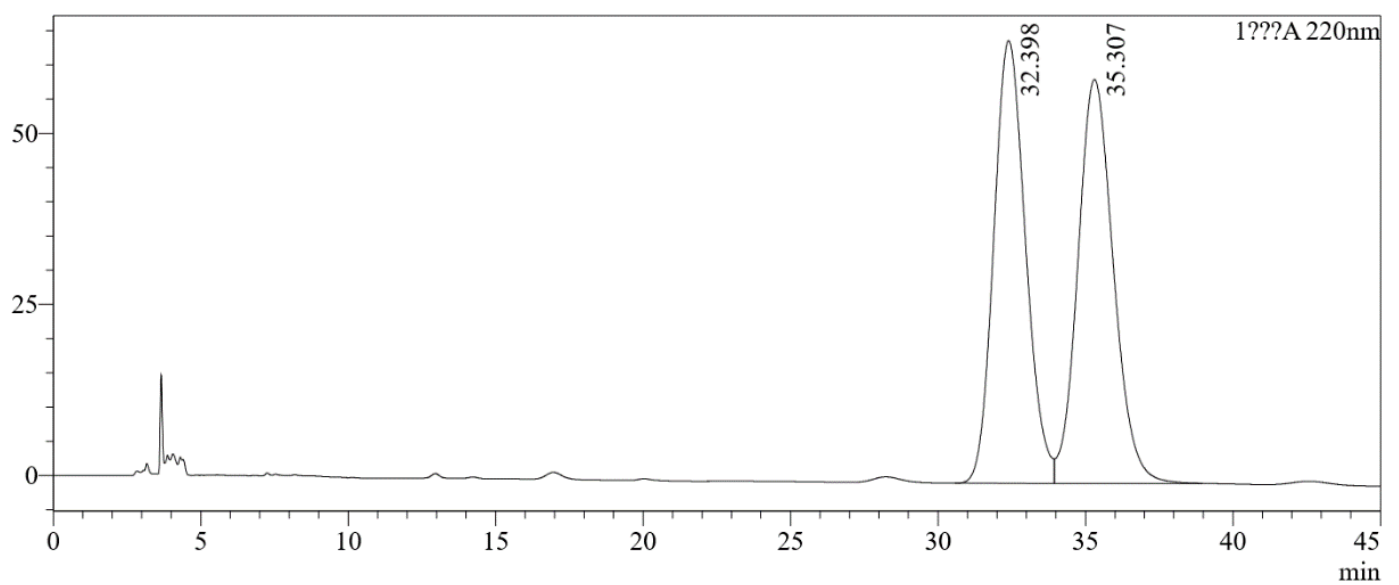

???A $220 \mathrm{~nm}$
\begin{tabular}{|r|r|r|r|r|}
\hline Peak\# & Ret. Time & Area & Height & \multicolumn{1}{|c|}{ Area $\%$} \\
\hline 1 & 32.398 & 4847695 & 64676 & 49.440 \\
\hline 2 & 35.307 & 4957504 & 59017 & 50.560 \\
\hline
\end{tabular}<smiles>[AlH2]NC(c1ccccc1)c1ccc2ccccc2c1</smiles>

$\mathrm{mV}$

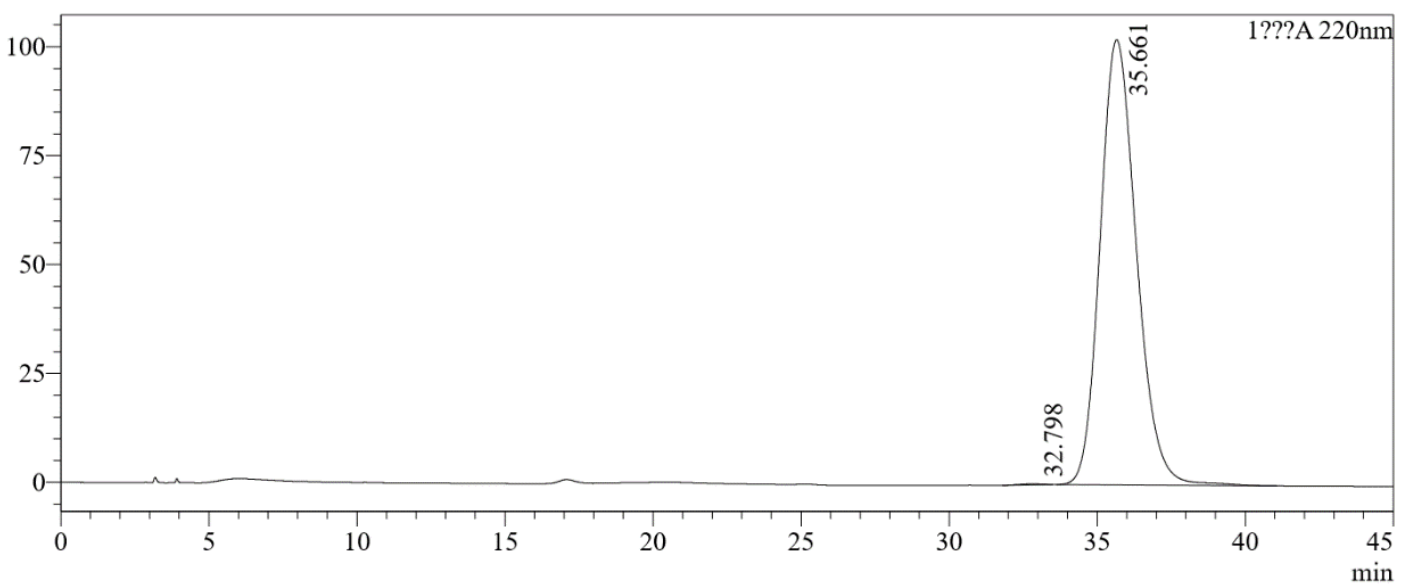

???A $220 \mathrm{~nm}$
\begin{tabular}{|r|r|r|r|r|}
\hline Peak\# & Ret. Time & Area & Height & \multicolumn{1}{c|}{ Area $\%$} \\
\hline 1 & 32.798 & 13147 & 240 & 0.150 \\
\hline 2 & 35.661 & 8737344 & 102165 & 99.850 \\
\hline
\end{tabular}




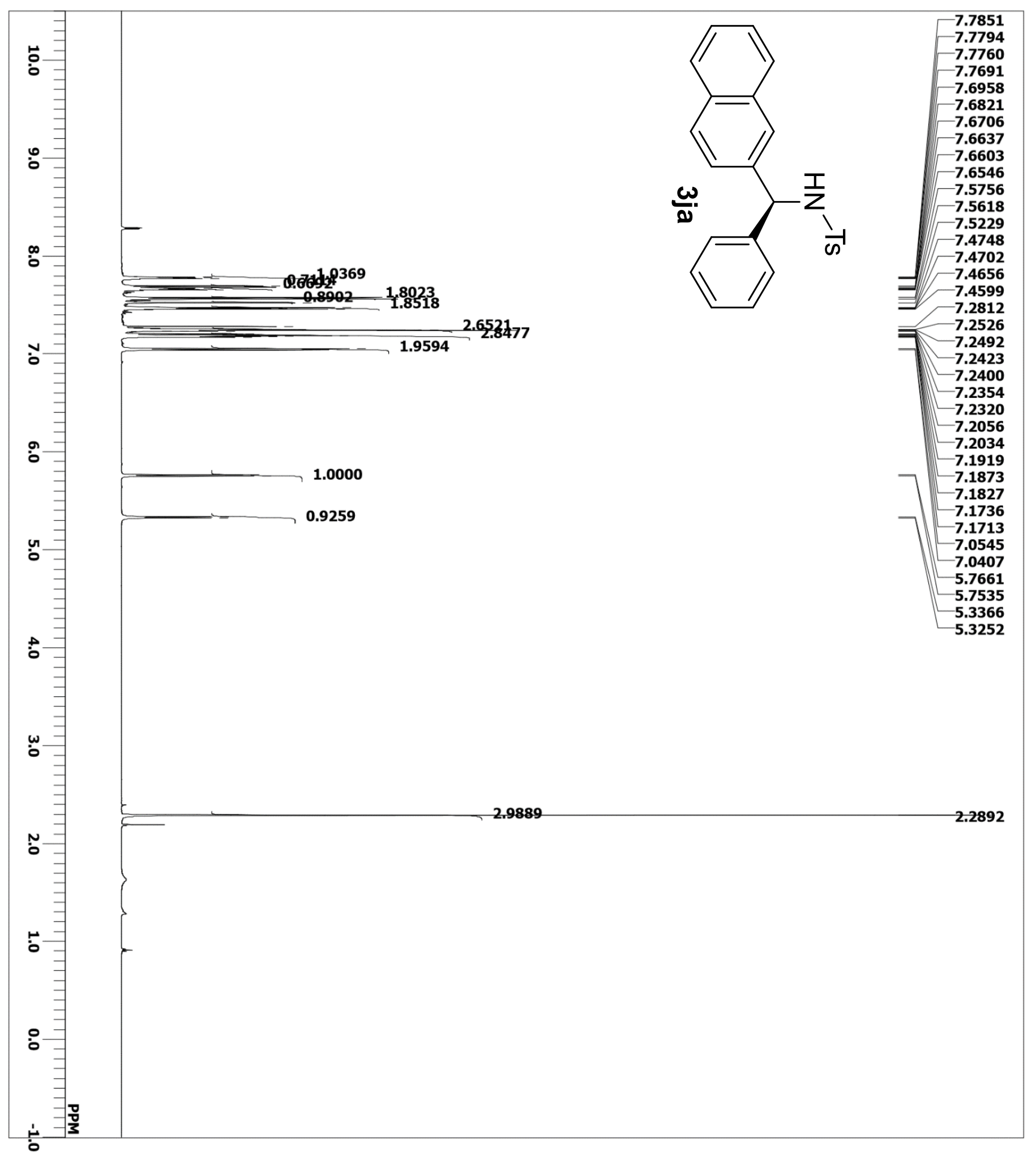




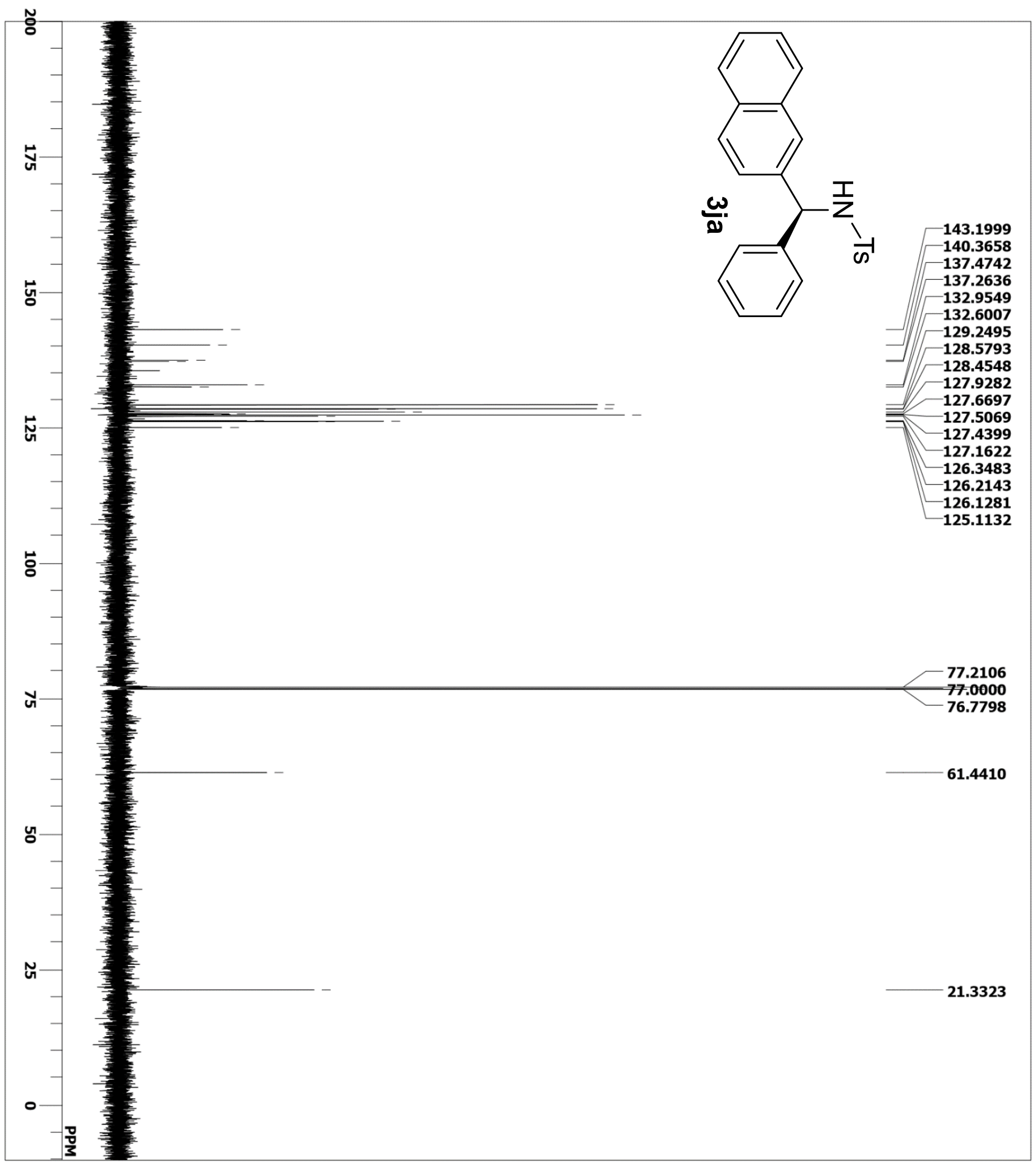




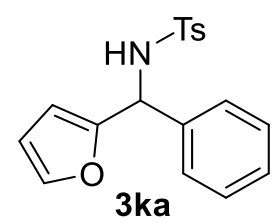

$\mathrm{mV}$

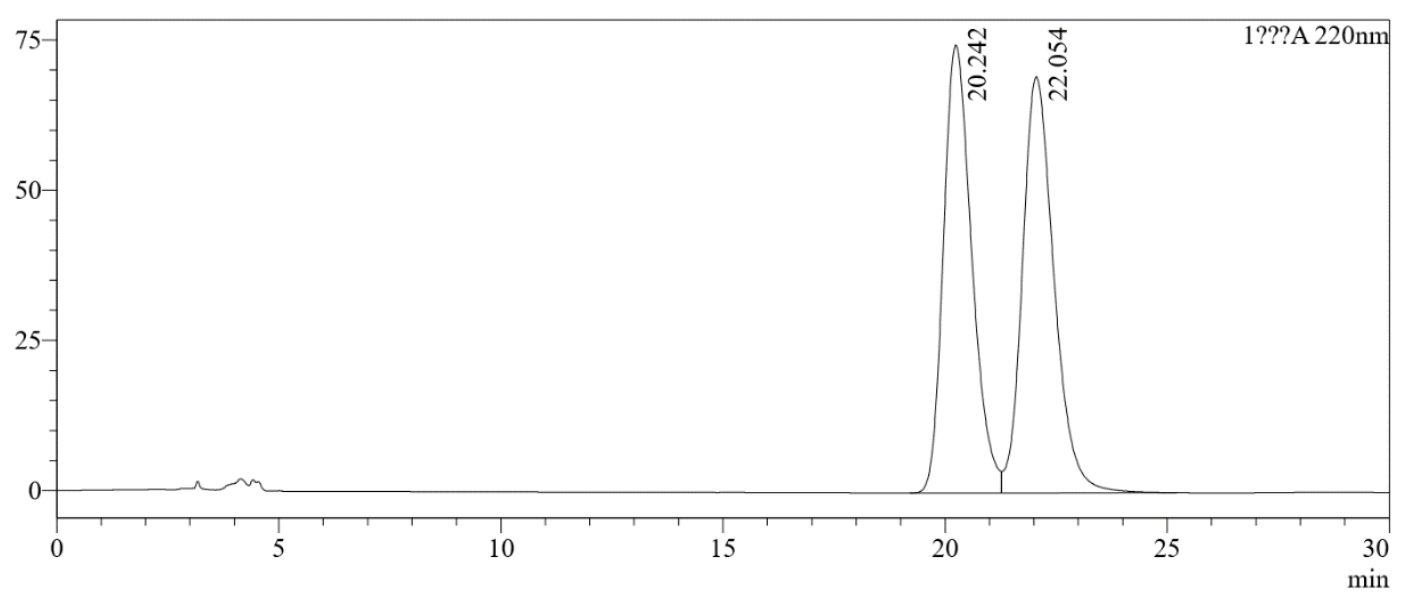

???A 220nm
\begin{tabular}{|r|r|r|r|r|}
\hline Peak\# & Ret. Time & Area & Height & \multicolumn{1}{c|}{ Area $\%$} \\
\hline 1 & 20.242 & 3312443 & 74574 & 49.146 \\
\hline 2 & 22.054 & 3427629 & 69253 & 50.854 \\
\hline
\end{tabular}

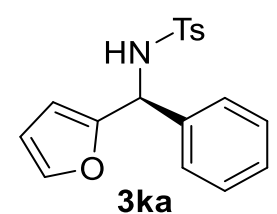

$\mathrm{mV}$

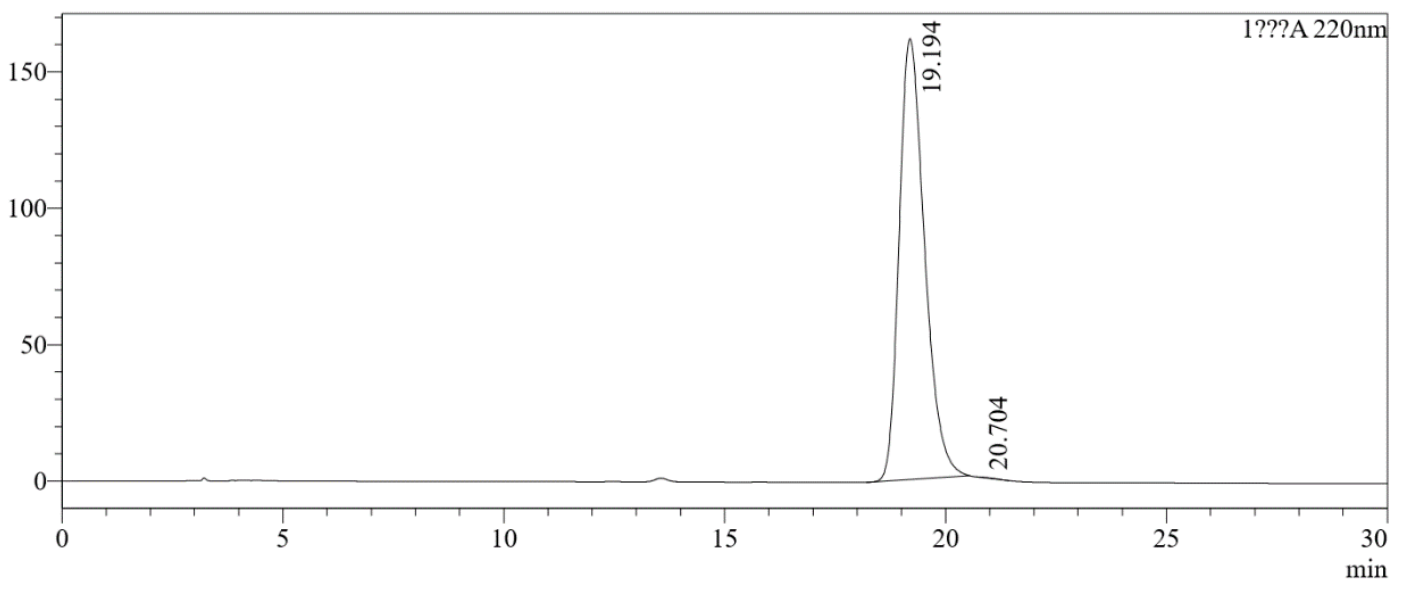

???A 220nm
\begin{tabular}{|r|r|r|r|r|}
\hline Peak\# & Ret. Time & Area & Height & \multicolumn{1}{|c|}{ Area $\%$} \\
\hline 1 & 19.194 & 6465812 & 161738 & 99.939 \\
\hline 2 & 20.704 & 3967 & 9 & 0.061 \\
\hline
\end{tabular}




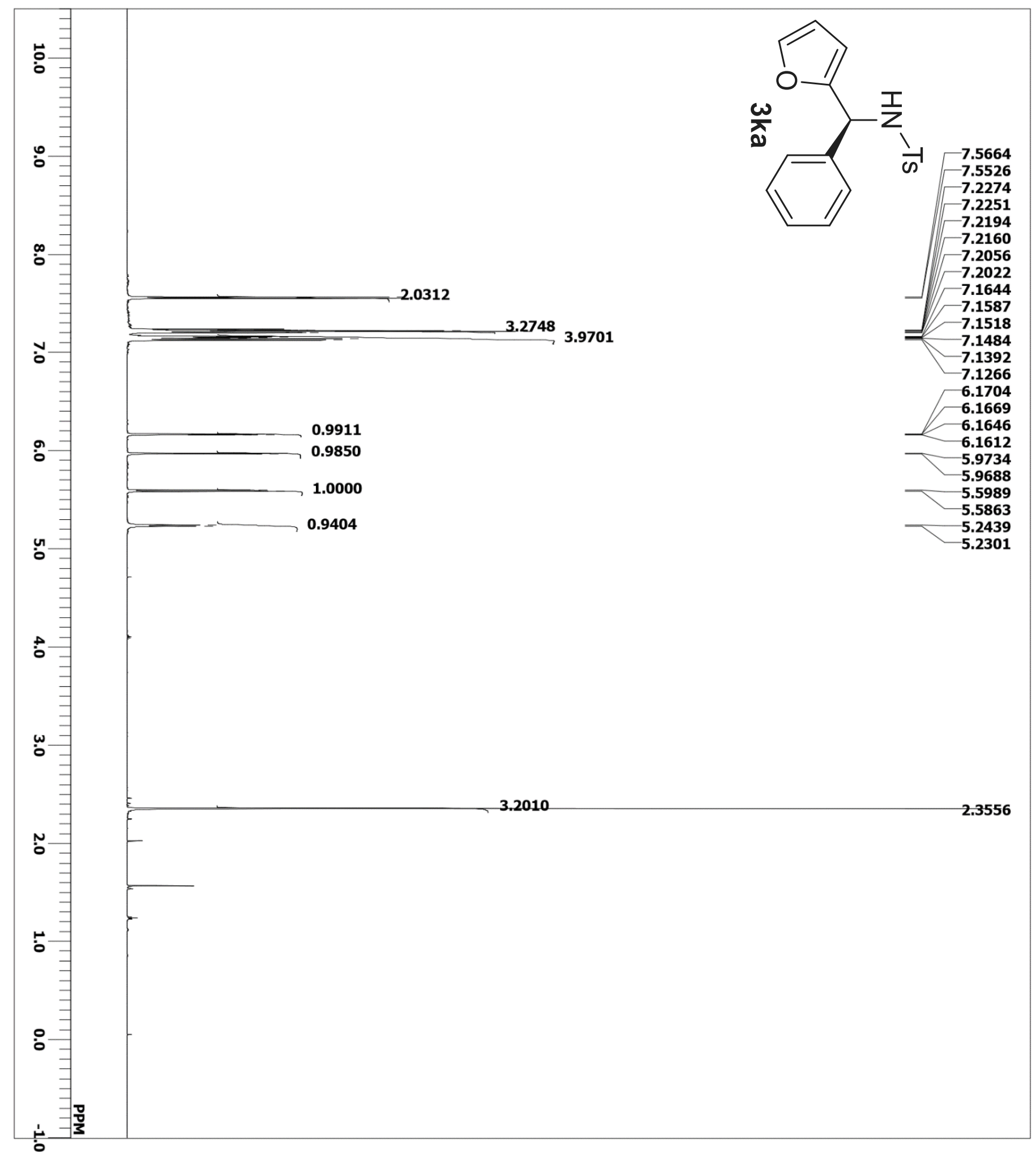




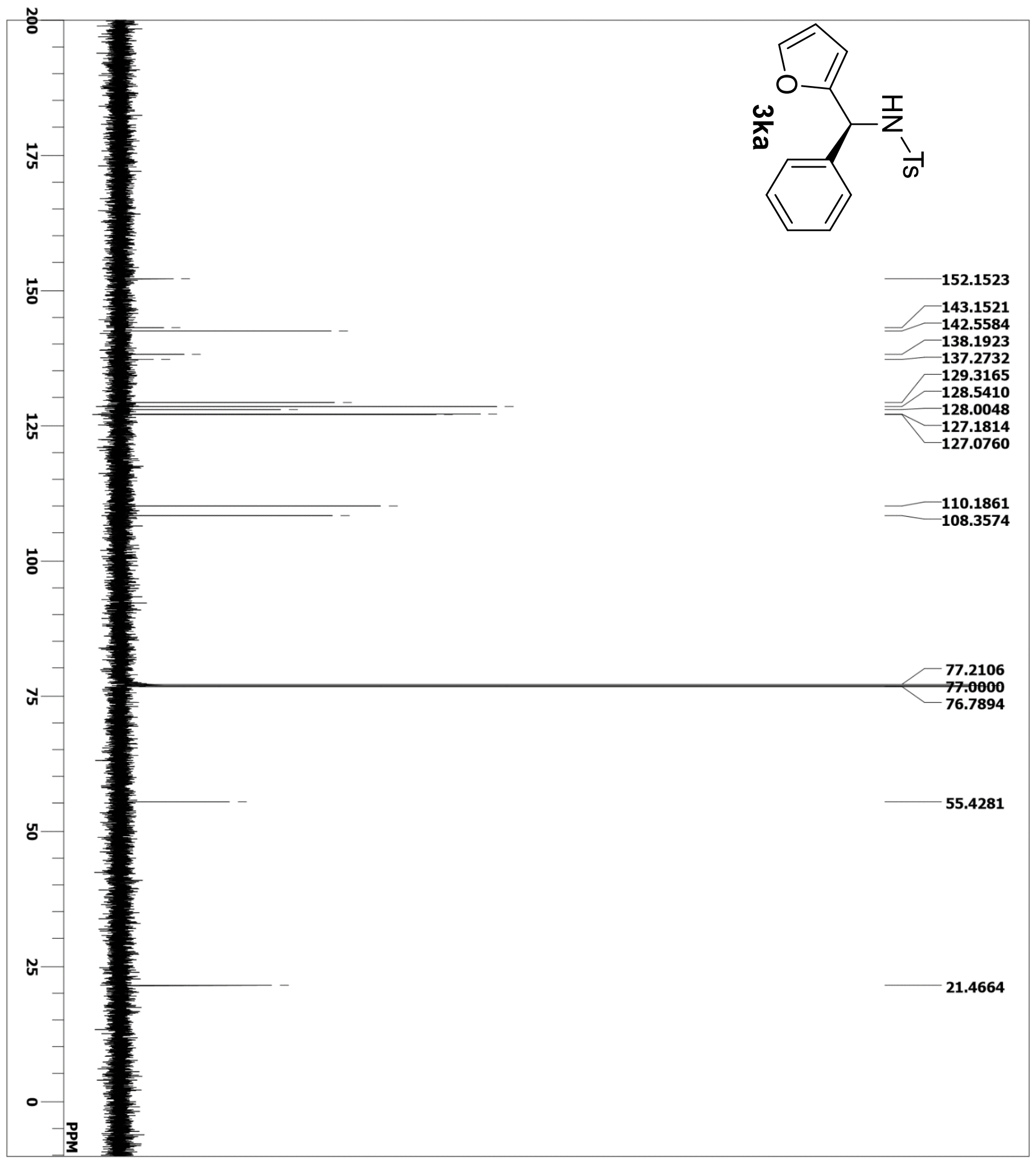


<smiles>FC(F)(F)NC(c1ccccc1)c1cccs1</smiles>

$\mathrm{mV}$

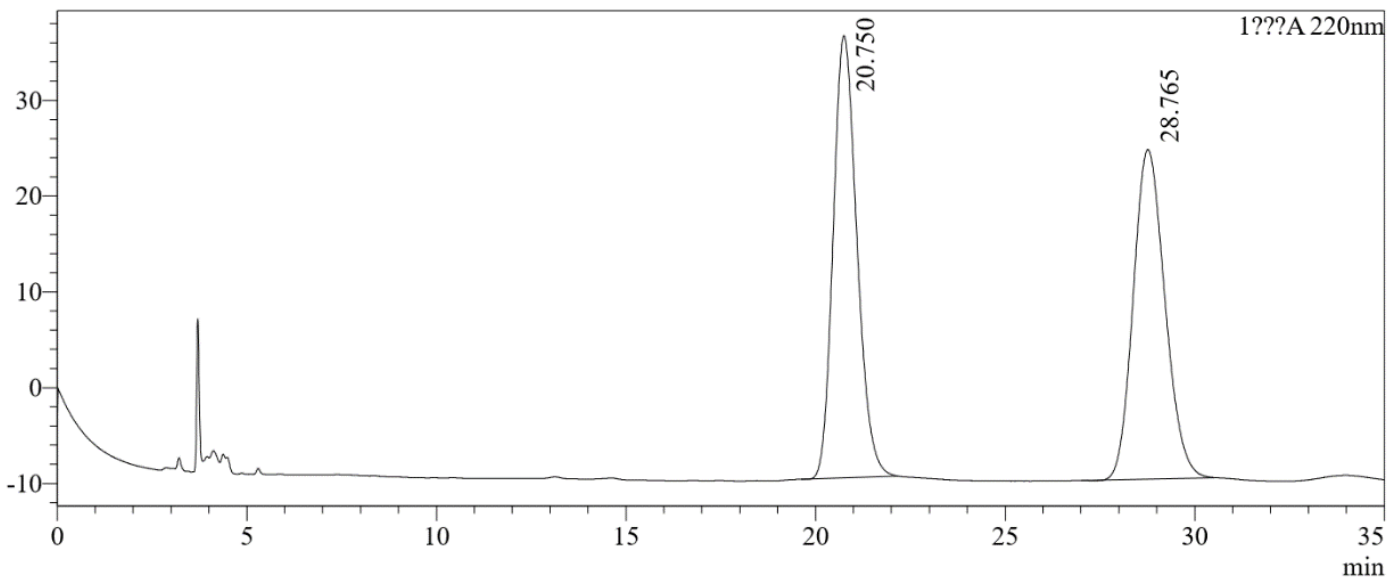

???A 220nm
\begin{tabular}{|r|r|r|r|r|}
\hline Peak\# & Ret. Time & \multicolumn{1}{l|}{ Area } & Height & \multicolumn{1}{c|}{ Area $\%$} \\
\hline 1 & 20.750 & 2009068 & 46175 & 50.103 \\
\hline 2 & 28.765 & 2000787 & 34423 & 49.897 \\
\hline
\end{tabular}

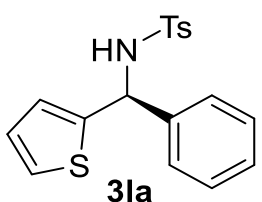

$\mathrm{mV}$

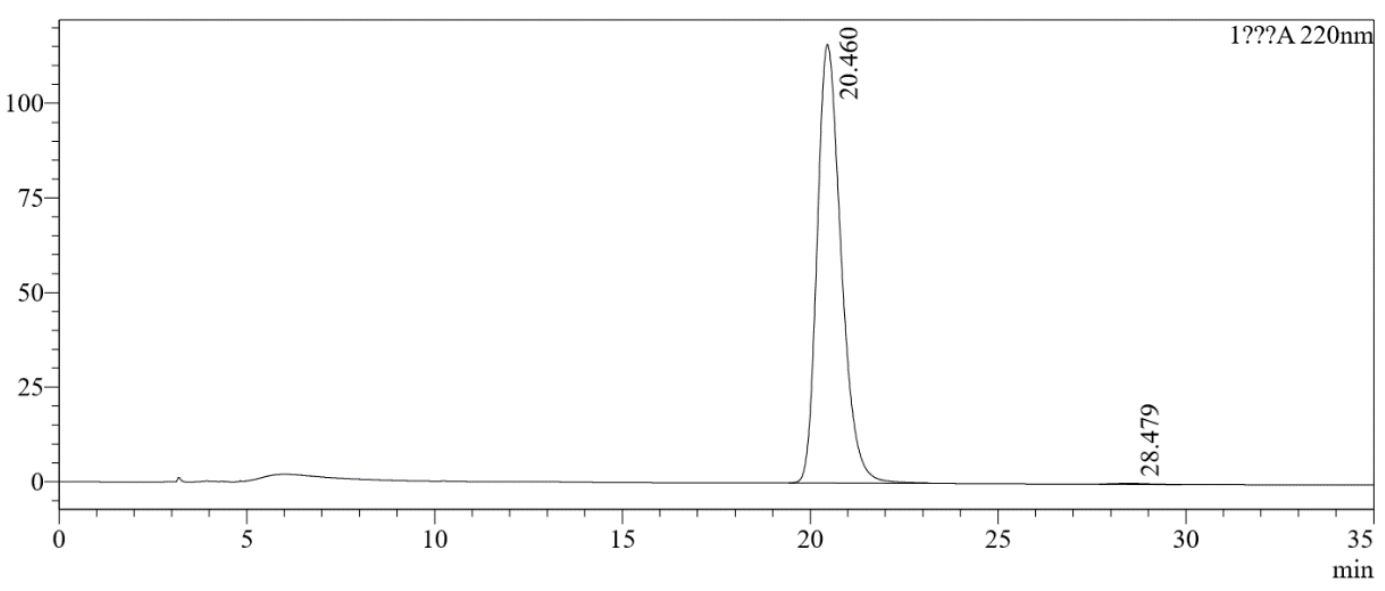

???A $220 \mathrm{~nm}$
\begin{tabular}{|r|r|r|r|r|}
\hline Peak\# & Ret. Time & \multicolumn{1}{|c|}{ Area } & Height & \multicolumn{1}{|c|}{ Area $\%$} \\
\hline 1 & 20.460 & 5149144 & 115902 & 99.749 \\
\hline 2 & 28.479 & 12948 & 227 & 0.251 \\
\hline
\end{tabular}




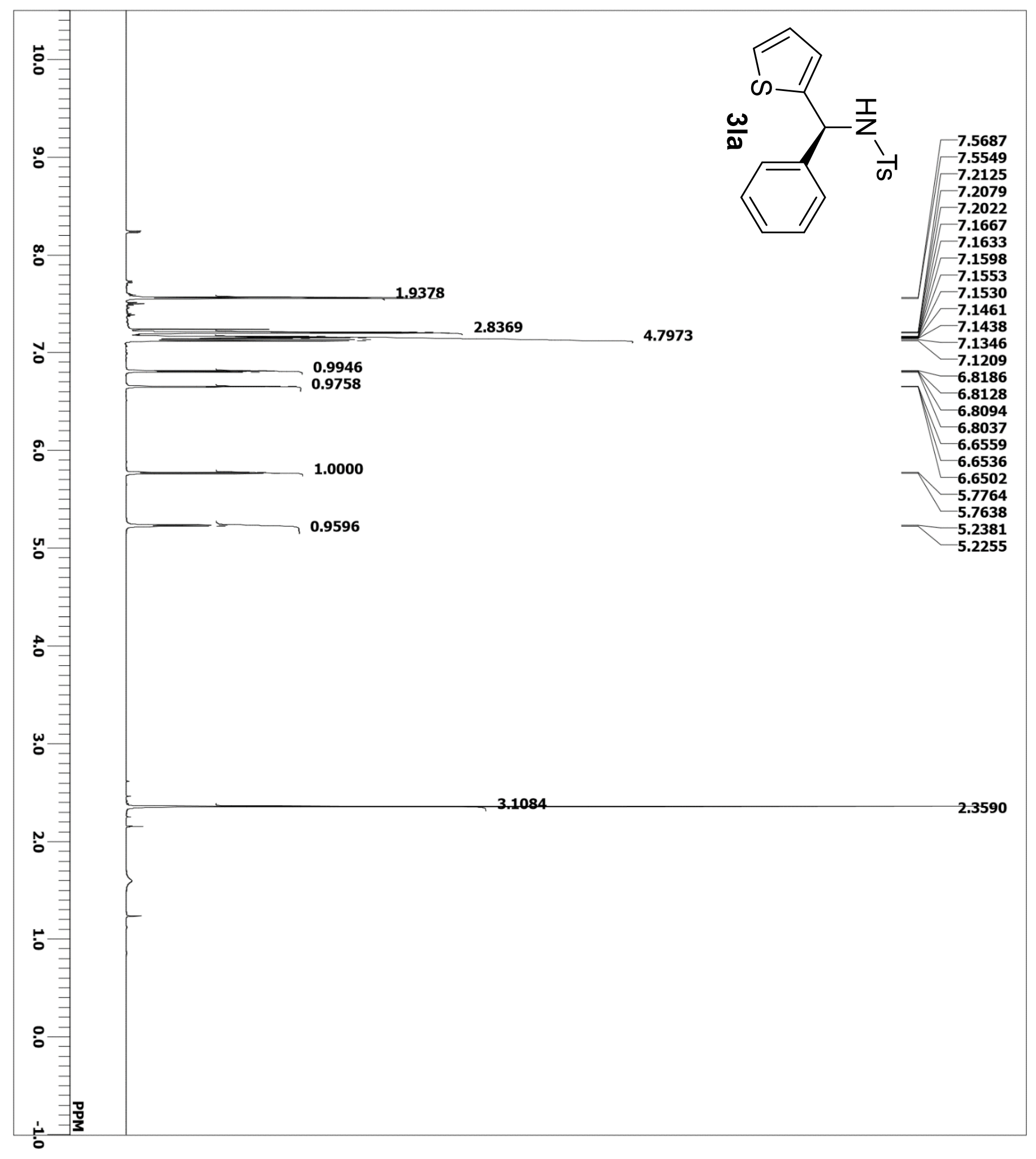




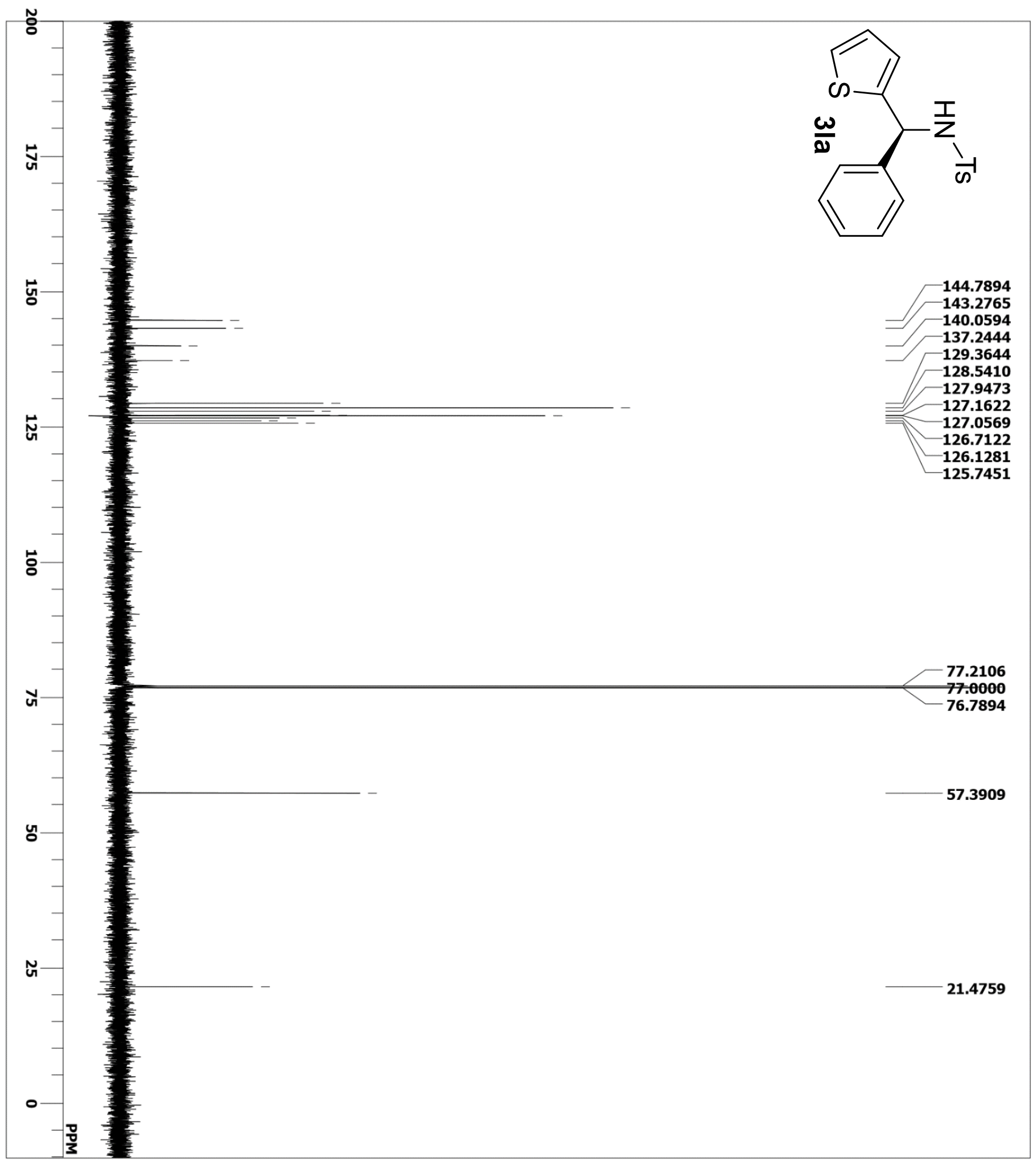




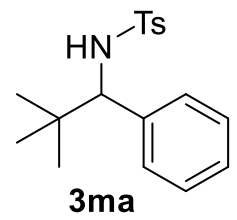

$\mathrm{mV}$

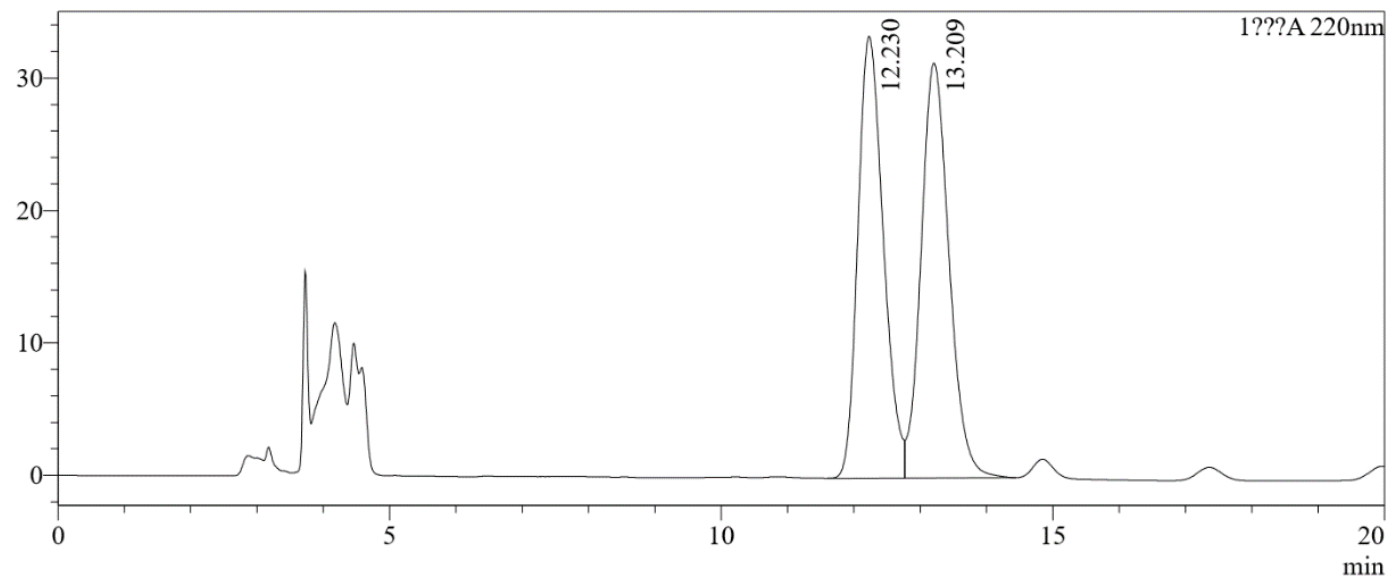

???A $220 \mathrm{~nm}$
\begin{tabular}{|r|r|r|r|r|}
\hline Peak\# & Ret. Time & Area & Height & \multicolumn{1}{|c|}{ Area $\%$} \\
\hline 1 & 12.230 & 892818 & 33363 & 49.230 \\
\hline 2 & 13.209 & 920748 & 31324 & 50.770 \\
\hline
\end{tabular}

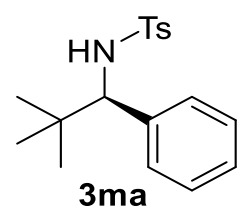

$\mathrm{mV}$

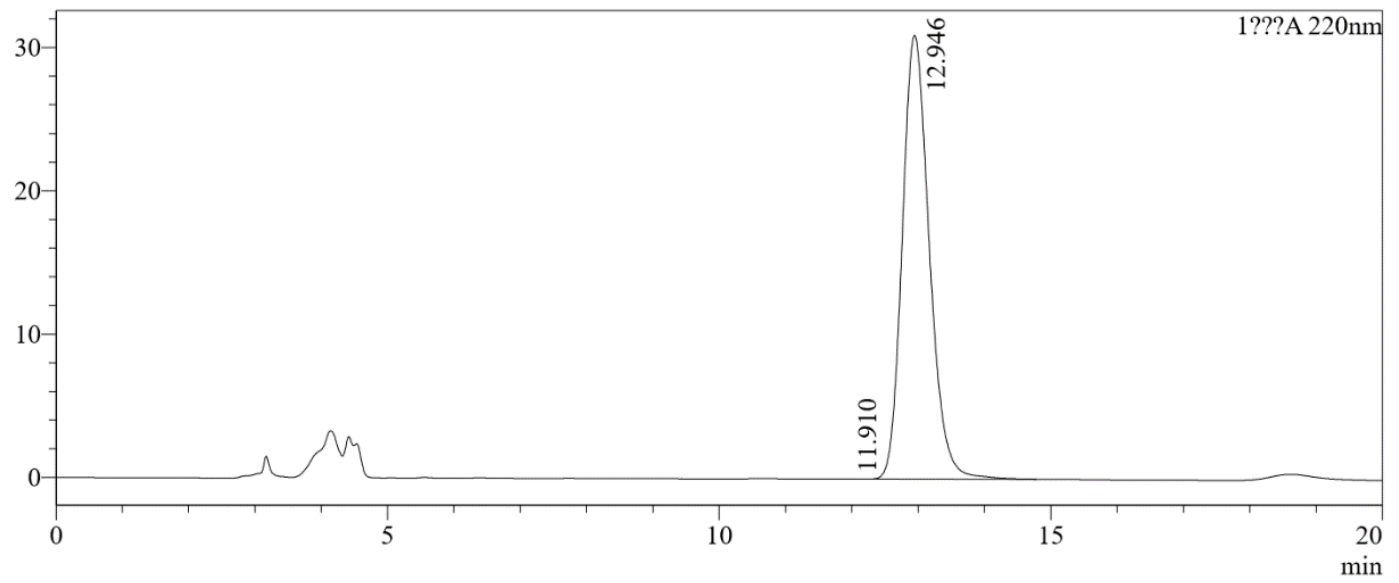

???A 220nm

\begin{tabular}{|r|r|r|r|r|}
\hline Peak\# & Ret. Time & \multicolumn{1}{|c|}{ Area } & \multicolumn{1}{|c|}{ Height } & \multicolumn{1}{|c|}{ Area\% } \\
\hline 1 & 11.910 & 167 & 10 & 0.019 \\
\hline 2 & 12.946 & 875771 & 30951 & 99.981 \\
\hline
\end{tabular}




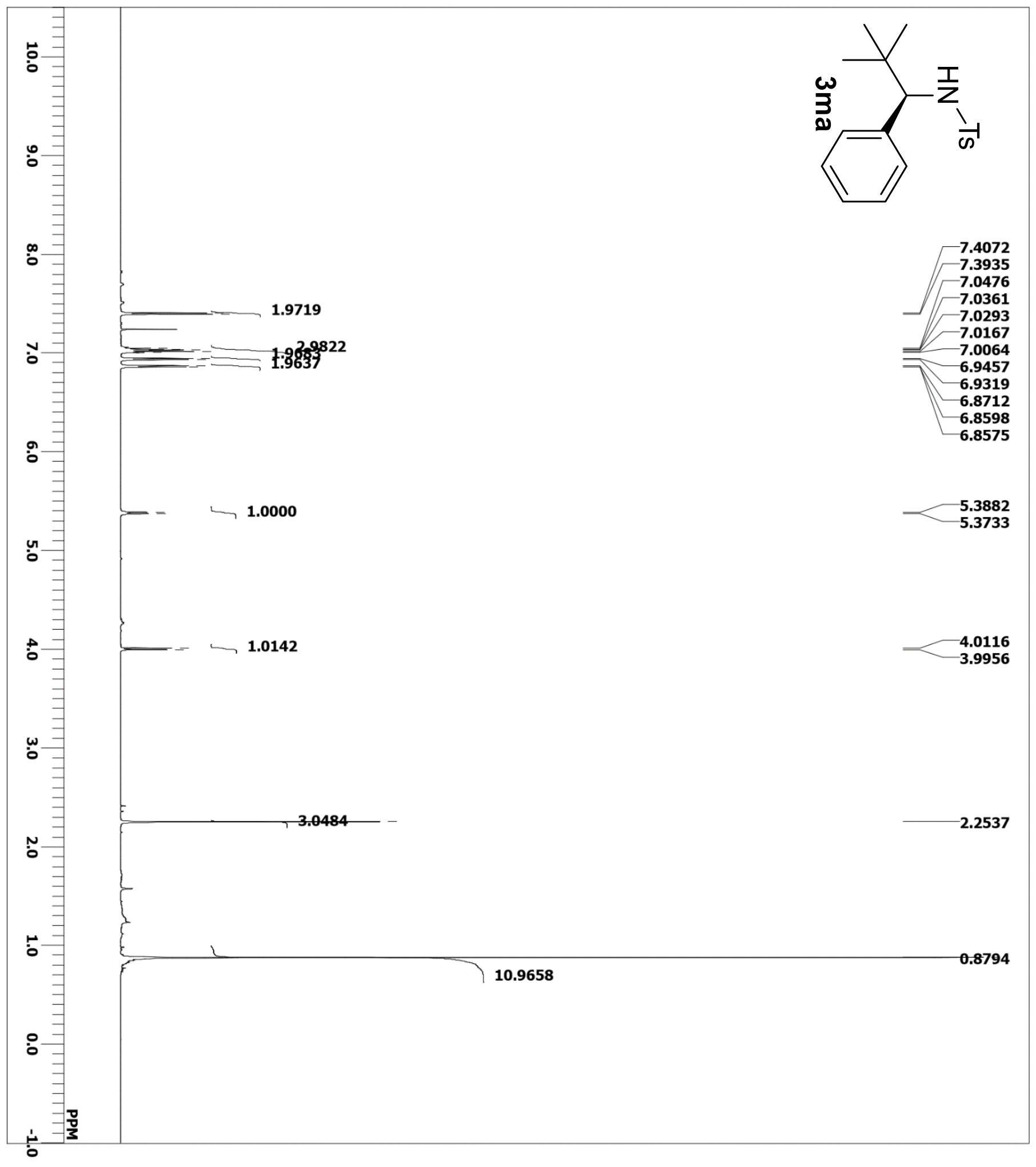




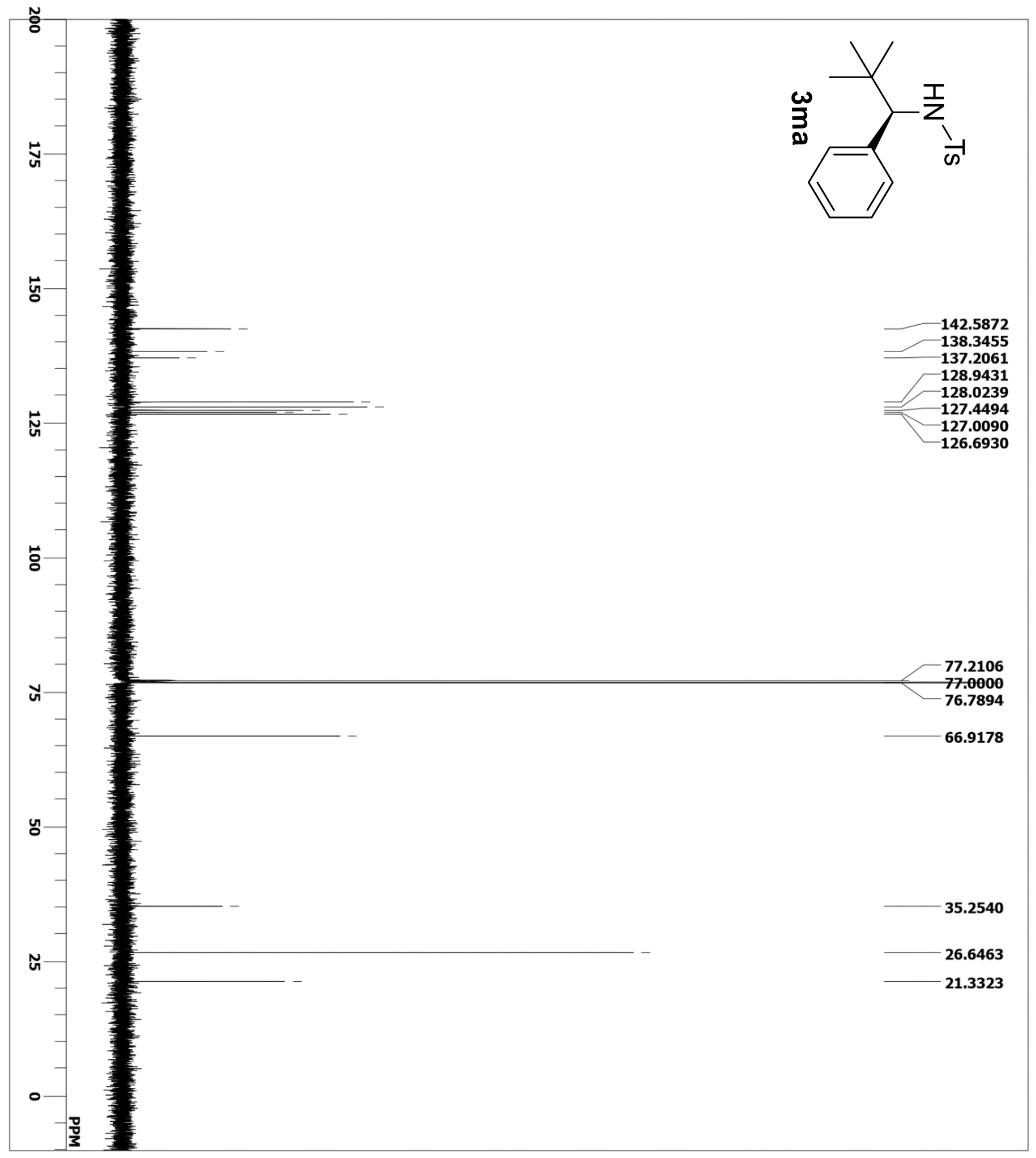


<smiles></smiles>

$\mathrm{mV}$

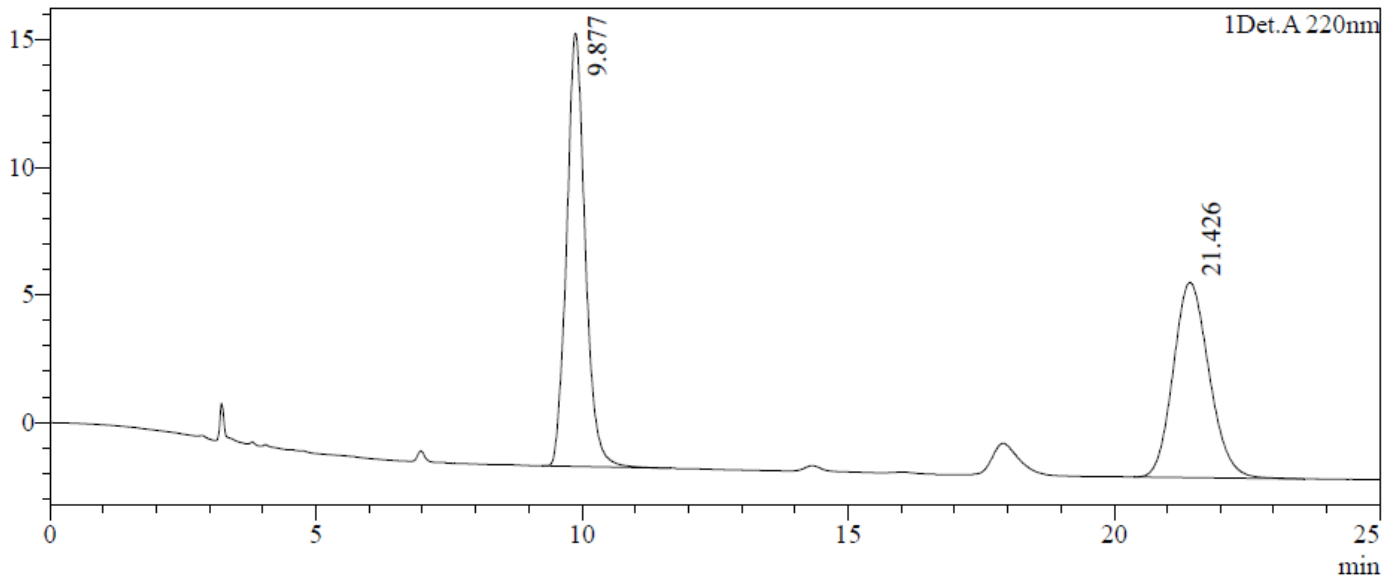

Det.A 220nm

\begin{tabular}{r|r|r|r|r|}
\hline Peak\# & Ret. Time & \multicolumn{1}{c|}{ Area } & Height & \multicolumn{1}{c|}{ Area\% } \\
\hline 1 & 9.877 & 412179 & 16962 & 53.435 \\
\hline 2 & 21.426 & 359181 & 7643 & 46.565 \\
\hline
\end{tabular}

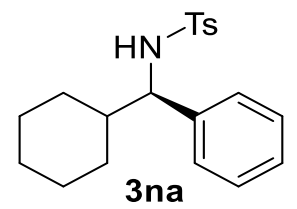

$\mathrm{mV}$

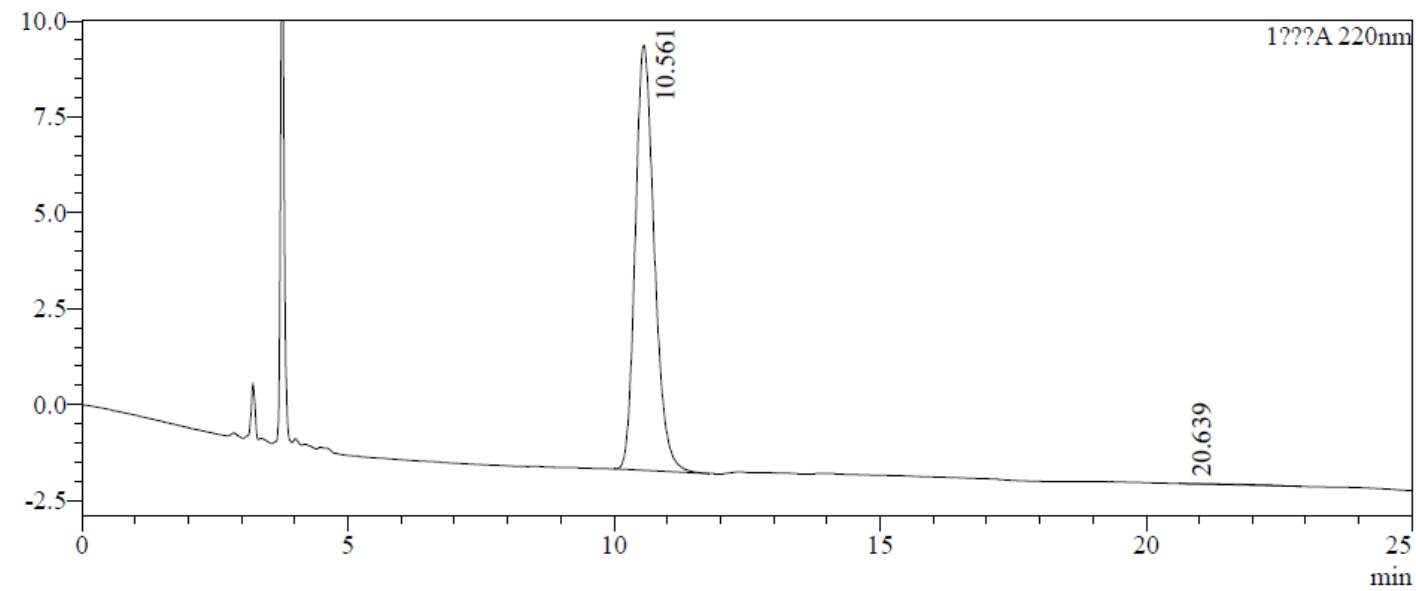

???A $220 \mathrm{~nm}$
\begin{tabular}{|r|r|r|r|r|}
\hline Peak\# & Ret. Time & \multicolumn{1}{c|}{ Area } & Height & \multicolumn{1}{|c|}{ Area $\%$} \\
\hline 1 & 10.561 & 276165 & 11085 & 99.642 \\
\hline 2 & 20.639 & 992 & 0 & 0.358 \\
\hline
\end{tabular}




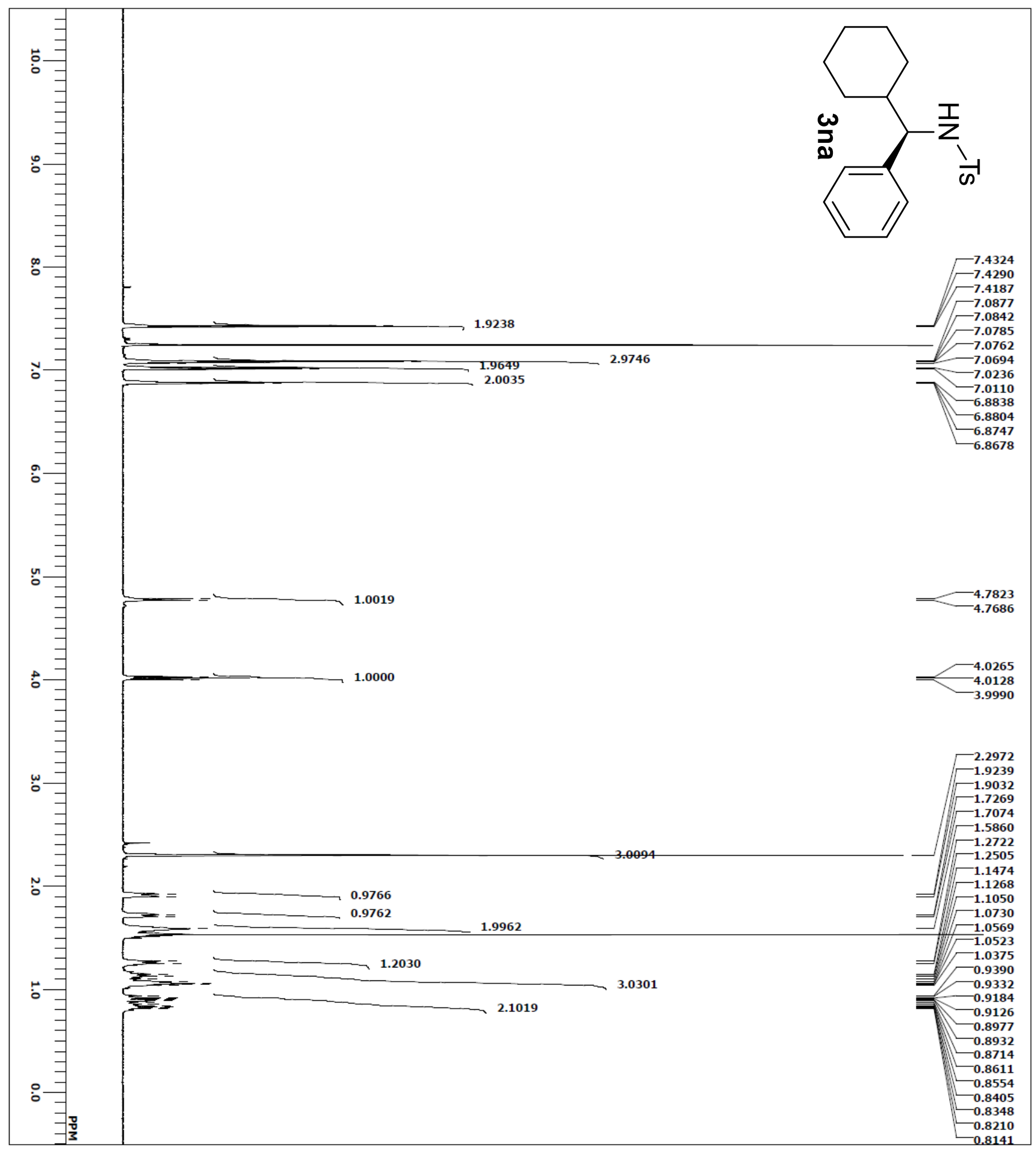




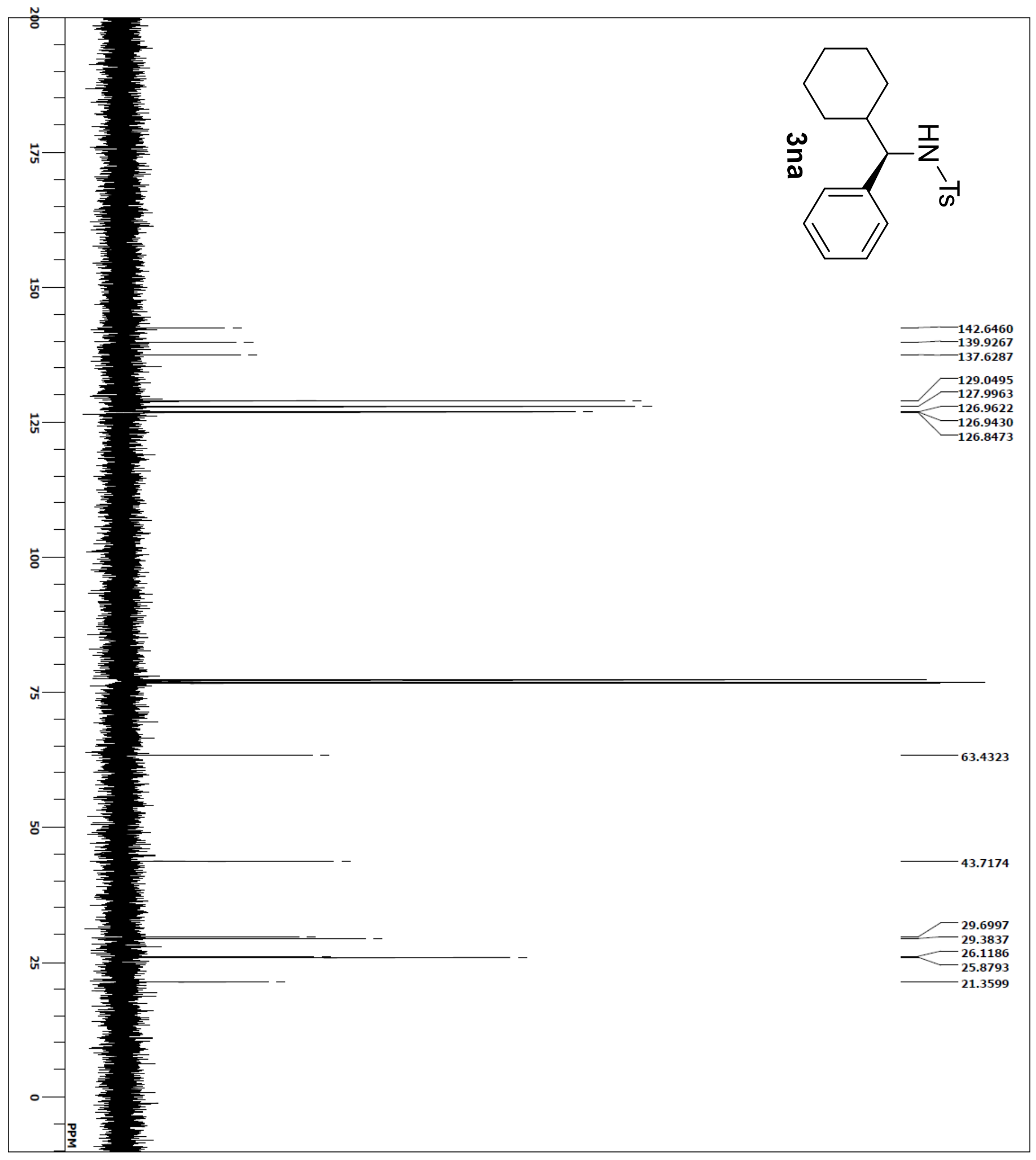




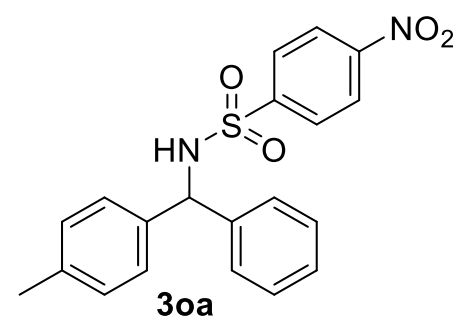

$\mathrm{mV}$
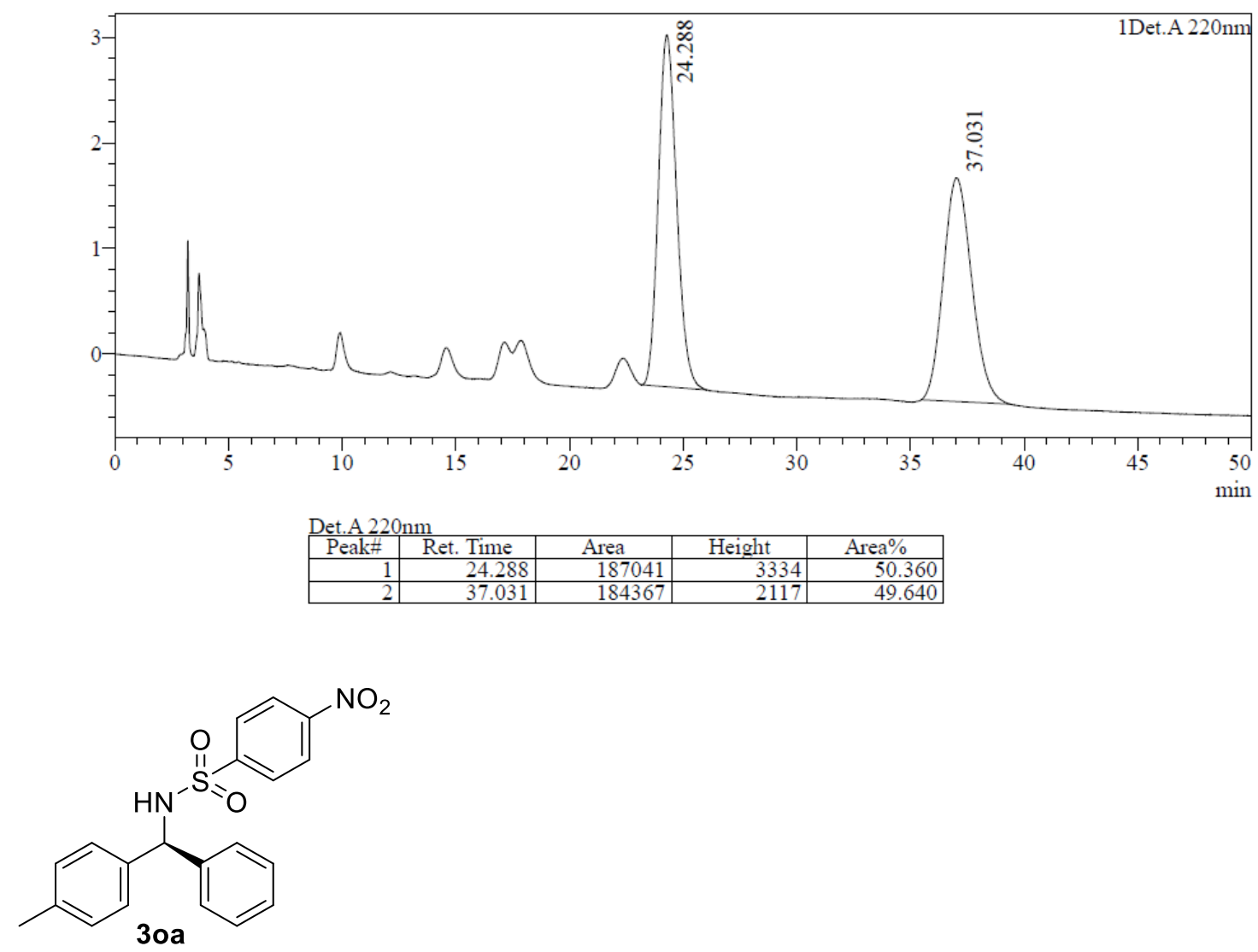

$\mathrm{mV}$

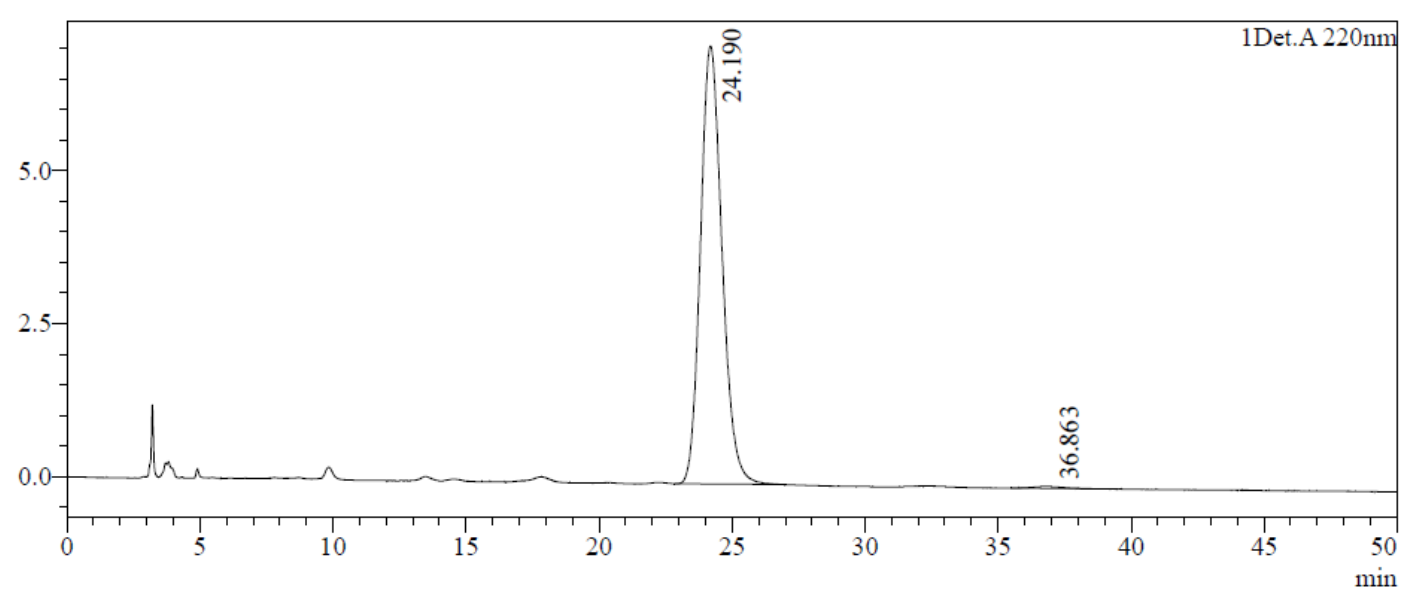

Det.A $220 \mathrm{~nm}$
\begin{tabular}{|r|r|r|r|r|}
\hline Peak\# & Ret. Time & Area & Height & \multicolumn{1}{|c|}{ Area $\%$} \\
\hline 1 & 24.190 & 404312 & 7152 & 99.402 \\
\hline 2 & 36.863 & 2432 & 31 & 0.598 \\
\hline
\end{tabular}




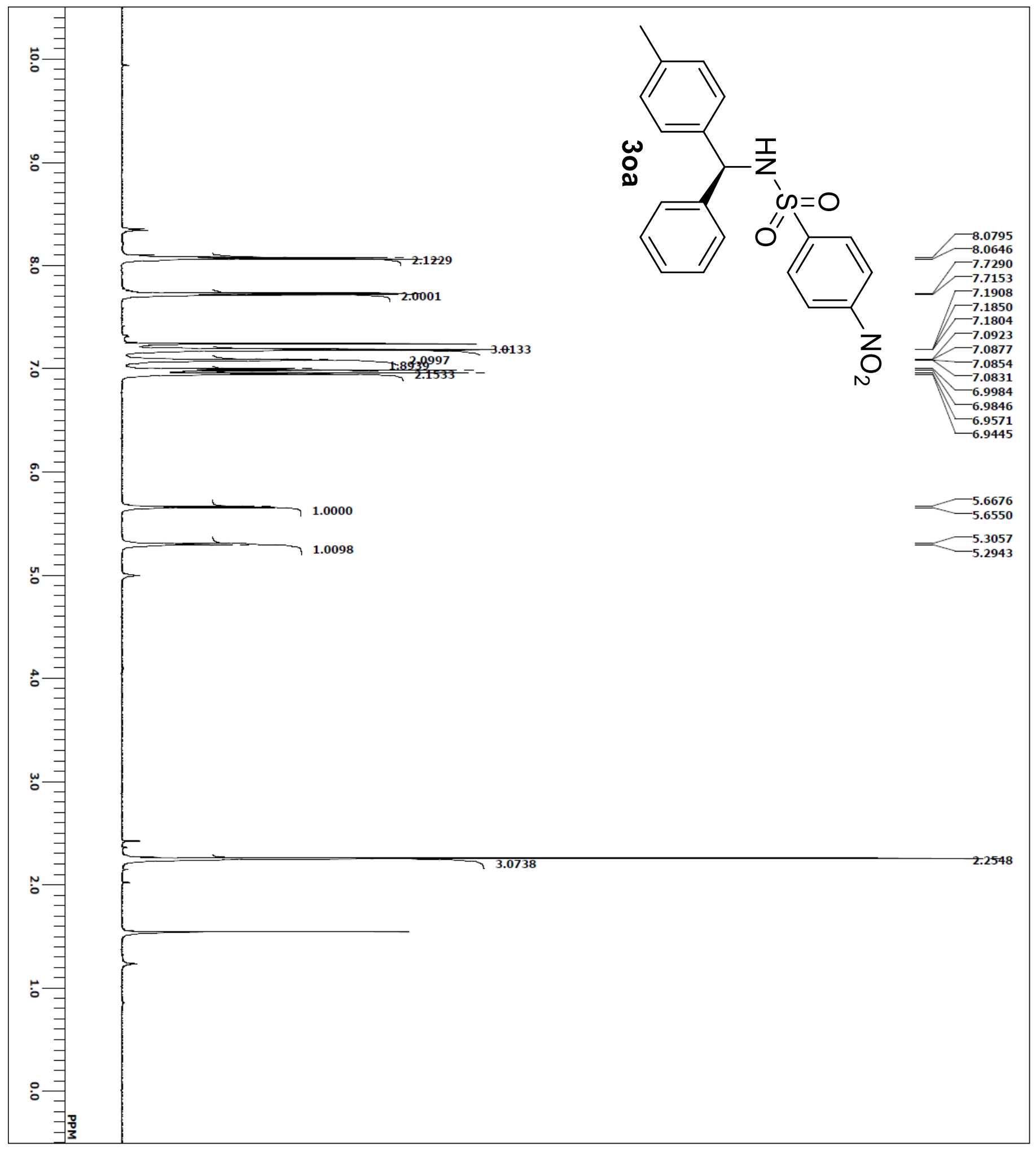




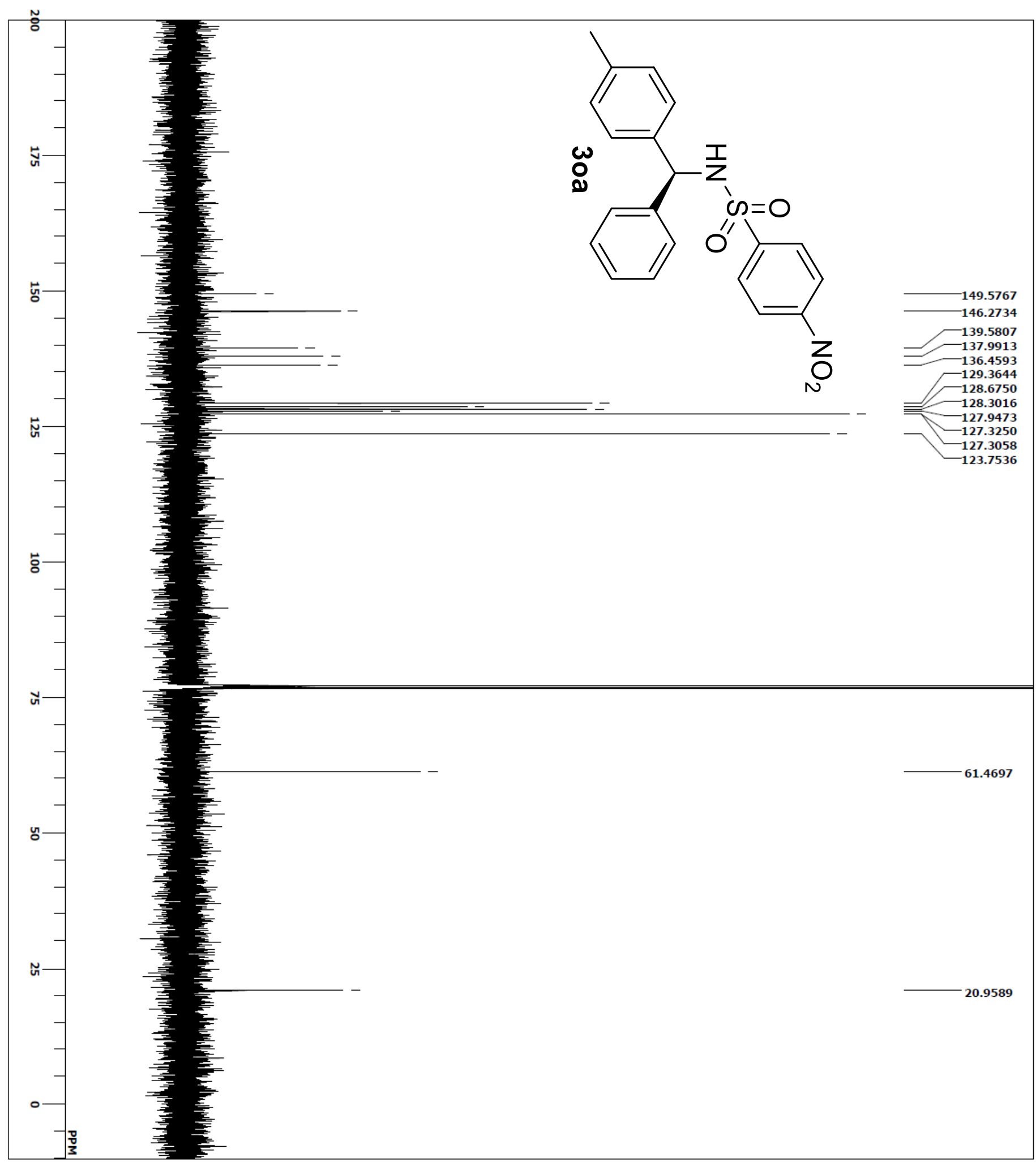

